EXTENDING THE SPEED RANGE OF A SWITCHED RELUCTANCE MOTOR USING A FAST DEMAGNETIZING TECHNIQUE

\author{
A Thesis \\ Presented to \\ The Graduate Faculty of the University of Akron
}

\author{
In Partial Fulfillment \\ of the Requirements for the Degree \\ Master of Science
}

Mohamad Abd Elmutalab

August, 2016 


\title{
EXTENDING THE SPEED RANGE OF A SWITCHED RELUCTANCE MOTOR USING A FAST DEMAGNETIZING TECHNIQUE
}

\author{
Mohamad Abd Elmutalab
}

Thesis

Approved:

Advisor

Dr. Yilmaz Sozer

Committee Member

Dr. Malik Elbuluk

Committee Member

Dr. Jose A. De Abreu-Garcia
Accepted:

Interim Department Chair

Dr. Joan Carletta

Interim Dean of the College

Dr. Donald Visco

Dean of the Graduate School

Dr. Chand Midha 


\begin{abstract}
The objective of this thesis is to improve the high speed range of a switched reluctance motor (SRM). An auto-transformer-like winding is proposed to achieve fast demagnetization of phase windings for better high speed performance.

The re-configured winding requires the development of a new power converter to drive the motor. The developed converter applies the DC bus voltage across a portion of the phase winding during the demagnetization time interval. Accordingly, a higher negative demagnetization voltage is applied, leading to a faster discharge rate of the phase excitation currents.

Theoretical analysis of the proposed motor is presented to validate the effectiveness of the proposed demagnetizing technique. The proposed concept is verified through simulation using Finite Element Analysis and experimental tests on a rewound motor. A detailed comparison between the simulation and experimental results of the original and the rewound motors is given to highlight the advantages and the disadvantages of the proposed concept.
\end{abstract}




\section{DEDICATION}

I dedicate my thesis work to my family, advisors, and friends throughout the journey of my master degree. 


\section{ACKNOWLEDGMENTS}

I would like to express my sincere gratitude to my advisor, Dr. Yilmaz Sozer, who was extremely supportive and encouraging during my graduate studies. His guidance and care exceeded the technical sides to encircle my social and personal life.

I wish to convey my gratitude and appreciation to Dr. Malik Elbuluk, for his help and guidance in the social and academic aspects during my study at The University of Akron. I also thank my committee member, Dr. Jose A. De Abreu-Garcia for the great technical and social experience I gained while working with him throughout my study.

I would like also to acknowledge my colleagues in the Alternative Energy and Electric Machines Laboratory for their generous help and support through the friendly atmosphere.

Last but not least, I would like to thank my family for their unconditional love and continuous support throughout my life. 


\section{TABLE OF CONTENTS}

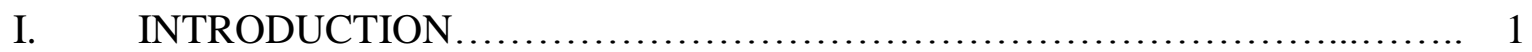

1.1. Switched Reluctance Motors.......................................... 1

1.2. SRM High Speed Characteristics.................................. 1

1.3. Research Motivation and Objectives.............................. 2

1.4. Thesis Organization............................................ 4

II. BACKGROUND AND RELATED WORK.............................. 5

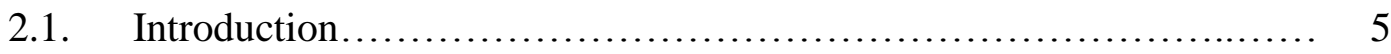

2.2. SRM Operation and Characteristics............................... 5

2.3. SRM Model.................................................. 9

2.4. SRM Control................................................. 12

2.5. SRM Speed Regions.............................................. 16

2.6. Related Work..................................................... 18

2.7. Conclusion........................................................... 20

III. THE PROPOSED THESIS WORK........................................ 21

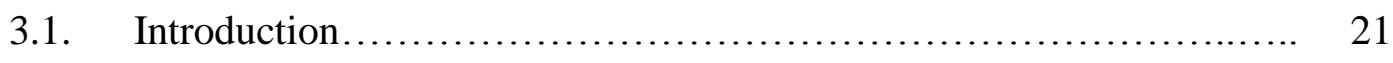

3.2. Split Phase Winding Scheme.................................... 21 
3.3. Proposed Power Converter........................................... 23

3.4. Analysis of The Motor Operation................................. 26

3.5. Conclusion ...................................................... 29

IV. SYSTEM MODELLING AND SIMULATION ......................... 30

4.1. Introduction ................................................. 30

4.2. SRM Finite Element Model...................................... 30

4.2.1. Conventional Machine Model................................ 31

4.2.2. The Proposed Motor Model...................................... $\quad 32$

4.3. Power Converter Model.................................................... 33

4.4. FEA Simulation Results............................................. 35

4.5. Simulation Results....................................................... 40

4.6. Leakage Inductance Simulation ................................... 44

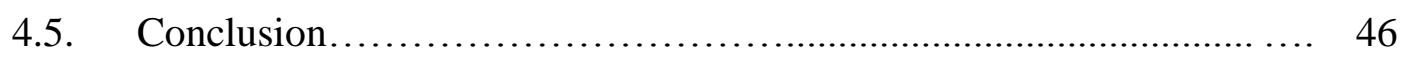

V. HARDWARE IMPLEMENTATION AND EXPERIMENTAL RESULTS ... 47

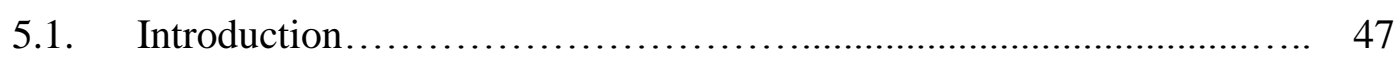

5.2. The Conventional Motor Setup................................ 47

5.3. The New Motor Development................................. 49 
5.4. Power Converter Design...................................... 52

5.4.1. The Design Requirements $\ldots \ldots \ldots \ldots \ldots \ldots \ldots \ldots \ldots \ldots \ldots \ldots . \ldots 2$

5.4.2. Printed Circuit Board (PCB) Design for the SRM Driver....... 55

5.5. The Experimental Setup................................... 58

5.6. Experimental Results.................................... 60

5.6.1. Unloaded Operation................................... 60

5.6.2. Loaded Operation........................................ 64

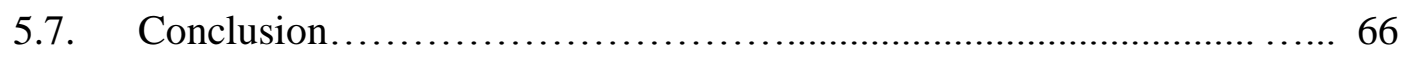

VI. CONCLUSION AND FUTURE WORK ................................ 67

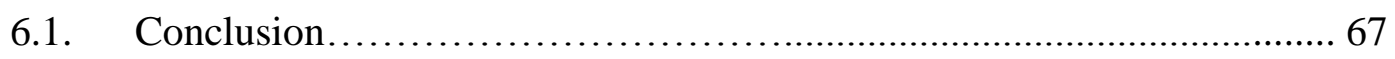

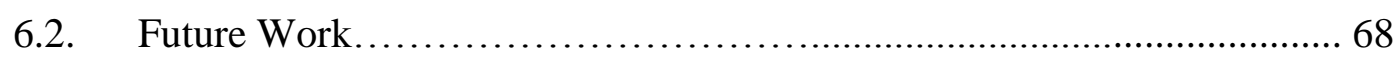

6.2.1. Low Speed Operation......................................... 68

6.2.2. Other Converter Topologies............................................... 68

VII. REFERENCES ....................................................... 67 


\section{LIST OF FIGURES}

FIGURES

PAGE

Figure 1.1: SRM asymmetric converter.......................................................... 3

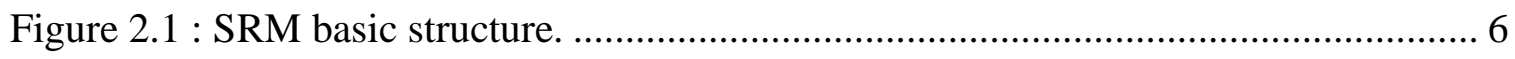

Figure 2.2 : Inductance profile for one SRM phase.............................................. 8

Figure 2.3 : SRM phase equivalent circuit model................................................. 9

Figure 2.4 : SRM magnetization mode for asymmetric converter............................... 14

Figure 2.5 : SRM freewheeling mode for asymmetric converter. ............................... 15

Figure 2.6 : SRM demagnetization mode for asymmetric converter........................... 16

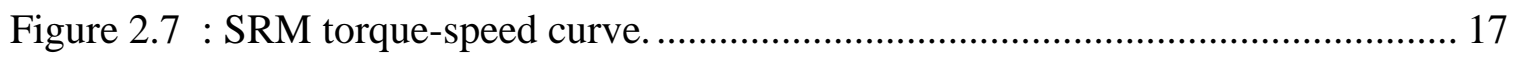

Figure 2.8 : Converter topology for fast demagnetization........................................ 19

Figure 3.1 : Autotransformer winding configuration in the stator pole......................... 22

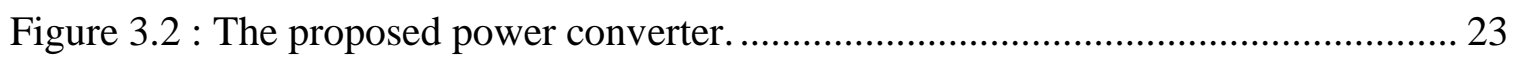

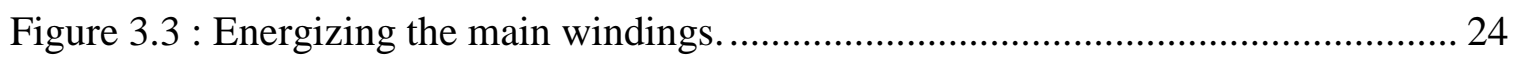

Figure 3.4 : Freewheeling mode for the new converter. .......................................... 25

Figure 3.5 : Demagnetization through the secondary windings. ................................. 26

Figure 4.1 : Conventional motor finite element model............................................ 31

Figure 4.2 : The new motor finite element model. .................................................... 33

Figure 4.3 : The inductance profile of the new motor model. ..................................... 33

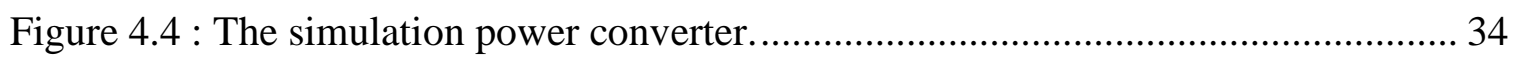

Figure 4.5 : The new motor power converter. ........................................................ 35

Figure 4.6 : Phase A current comparison at low speed (500 rpm) ............................... 36

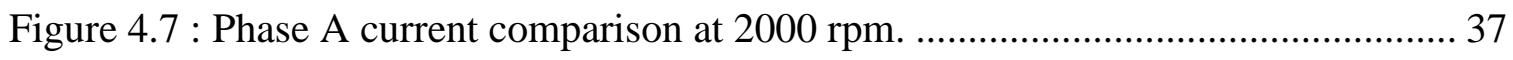




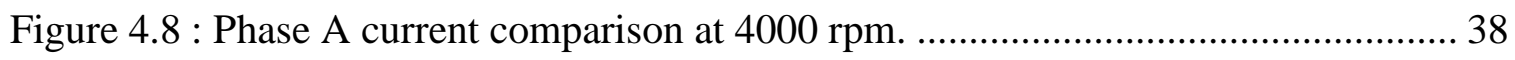

Figure 4.9 : Phase A voltages comparison at $2000 \mathrm{rpm}$........................................... 39

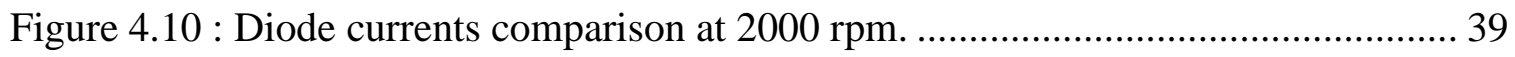

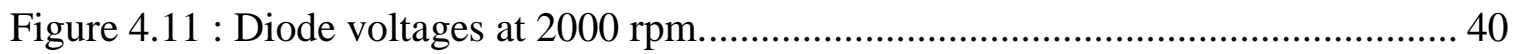

Figure 4.12 : Torque-speed curves comparison. .................................................. 41

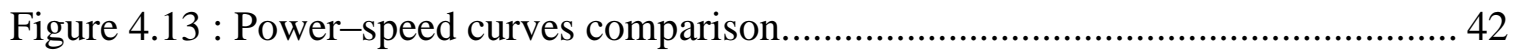

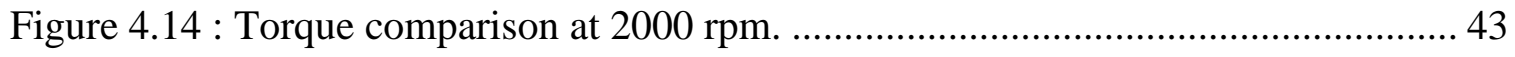

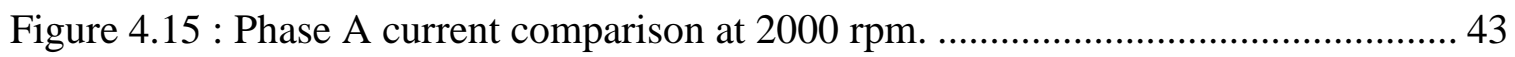

Figure 4.16 : Primary windings voltage for ideal coupling. ...................................... 44

Figure 4.17 : Primary windings voltage for non-ideal coupling................................. 45

Figure 4.18 : S2 Voltage for non-ideal coupling. .................................................... 45

Figure 4.19 : S1 Voltage for ideal and non-ideal coupling........................................ 46

Figure 5.1 : Conventional SRM experimental setup............................................ 48

Figure 5.2 : Phase currents of a conventional SRM operating at $500 \mathrm{rpm} . . . \ldots \ldots \ldots \ldots \ldots \ldots . . . . . . . . .49$

Figure 5.3 : Winding connection of the new SRM................................................. 50

Figure 5.4 : The new windings configuration of phase B...................................... 51

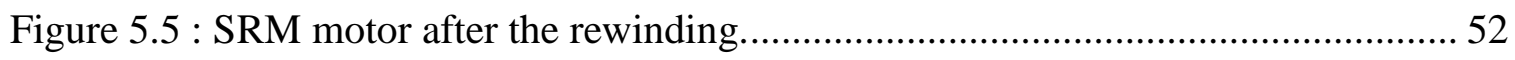

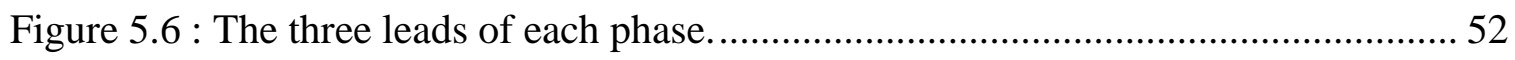

Figure 5.7 : The block diagram of the SRM driver circuit.. .................................... 54

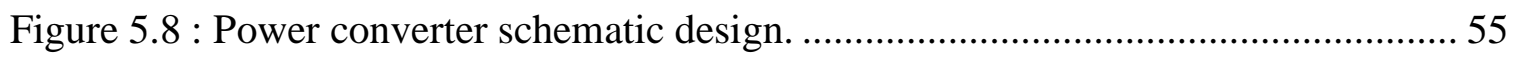

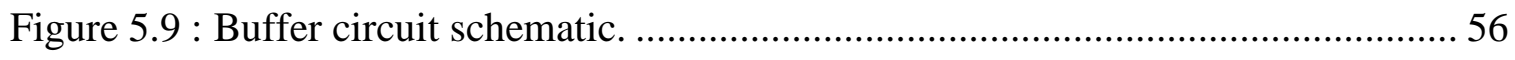

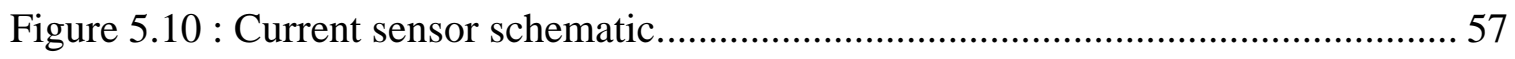

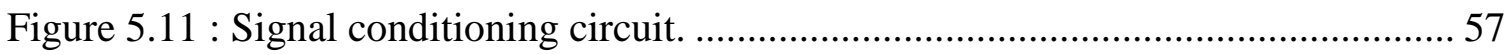


Figure 5.12 : Gate driver schematic. ................................................................ 58

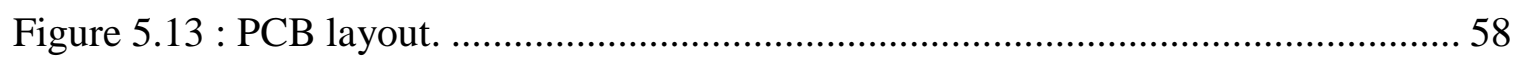

Figure 5.14 : The power converter and the new SRM............................................ 59

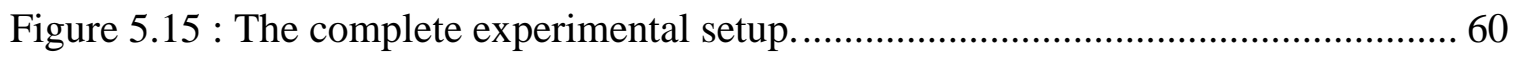

Figure 5.16 : Fast demagnetization current for the unloaded operation. ........................ 61

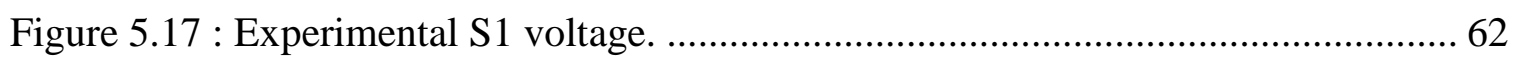

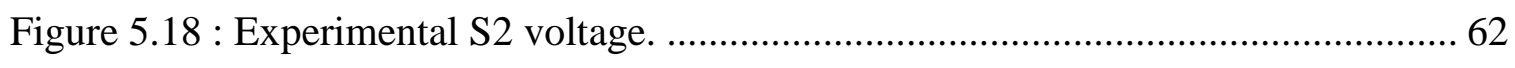

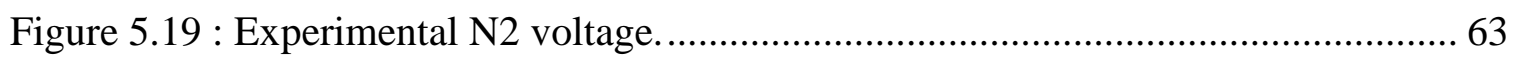

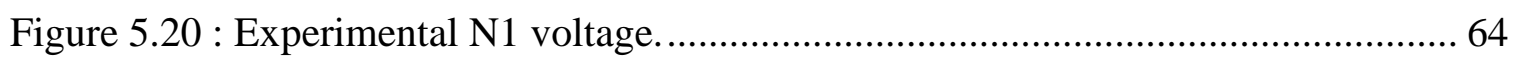

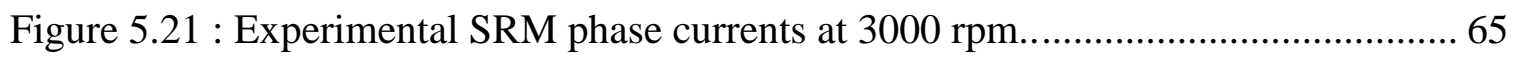




\section{LIST OF TABLES}

TABLES

PAGE

Table 4.1: Finite element SRM model parameters ................................................. 32

Table 4.2 : Turn-on and turn-off angle comparison................................................. 42

Table 5.1 : Finite element model and actual setup torque comparison for different operating conditions................................................................. 48

Table 5.2 : New motor torque production at high speeds....................................... 65

Table 5.3 : Experimental turn-on and turn-off angle comparison. ................................ 65 


\section{CHAPTER I}

\section{INTRODUCTION}

\subsection{Switched Reluctance Motors}

The switched reluctance motor (SRM), introduced in the mid-1800's, was first invented by Davidson for the Glasgow-Edinburgh railway system [1]. Due to their inherent advantageous features, such as high efficiency, robustness, and fault tolerance, SRMs have gained interest in many applications in the automotive industry, wind power generation, and aircraft starter-generator sets [2] - [3]. The recent development of power electronics further increased the interest of utilizing SRM drives. The main disadvantages of SRMs are torque ripple and acoustic noise, resulting from the inherent

doubly salient structure of the machine [4]. The broad expansion of SRMs is limited by these disadvantages, along with their control complexity [5].

The work proposed in this thesis tries to address one of the major drawbacks of SRMs encountered at high speeds; namely, their short demagnetization time.

\subsection{SRM High Speed Characteristics}

The performance of electric motors differs from one loading condition to another. The maximum speed and the associated electromagnetic torque produced at that speed define the motor operational boundaries. 
SRM is a synchronous motor in which the electrical excitation is governed by the motor's mechanical parameters in terms of the motor speed and the required electromagnetic torque. The high mechanical speed of the motor restrains the electrical current control and hence decreases both the efficiency and the energy conversion capabilities of the electric motor.

In order to ensure continuous production of motoring torque, the excitation current should be driven into the machine phases sequentially in a way that maximizes positive torque production while avoiding the generation of negative or breaking torque [6]. For SRM operation at different speeds, the turn-on and turn-off angles of the phase's excitation are determined based on two considerations; namely, the maximum possible positive torque production for motoring and the required time duration for the excitation current to be discharged, or demagnetized.

Clearly, improving the current controllability at high speeds will enhance the machine efficiency, thereby making the machine a good choice for a vast number of high speed applications in the automotive and perhaps other industries.

\subsection{Research Motivation and Objectives}

The demagnetization time duration is very critical as the phase current needs to be driven to zero before the rotor pole enters into the negative torque production region. The asymmetric converter, a typical topology used with SRMs, consists of two switches and two diodes per phase, as shown in Fig. 1.1 [7]. The two switches are responsible for controlling the driving current for each phase; the two diodes are required to provide a path for free-wheeling and for the discharge current to be pushed back to the converter DC bus [7]. 


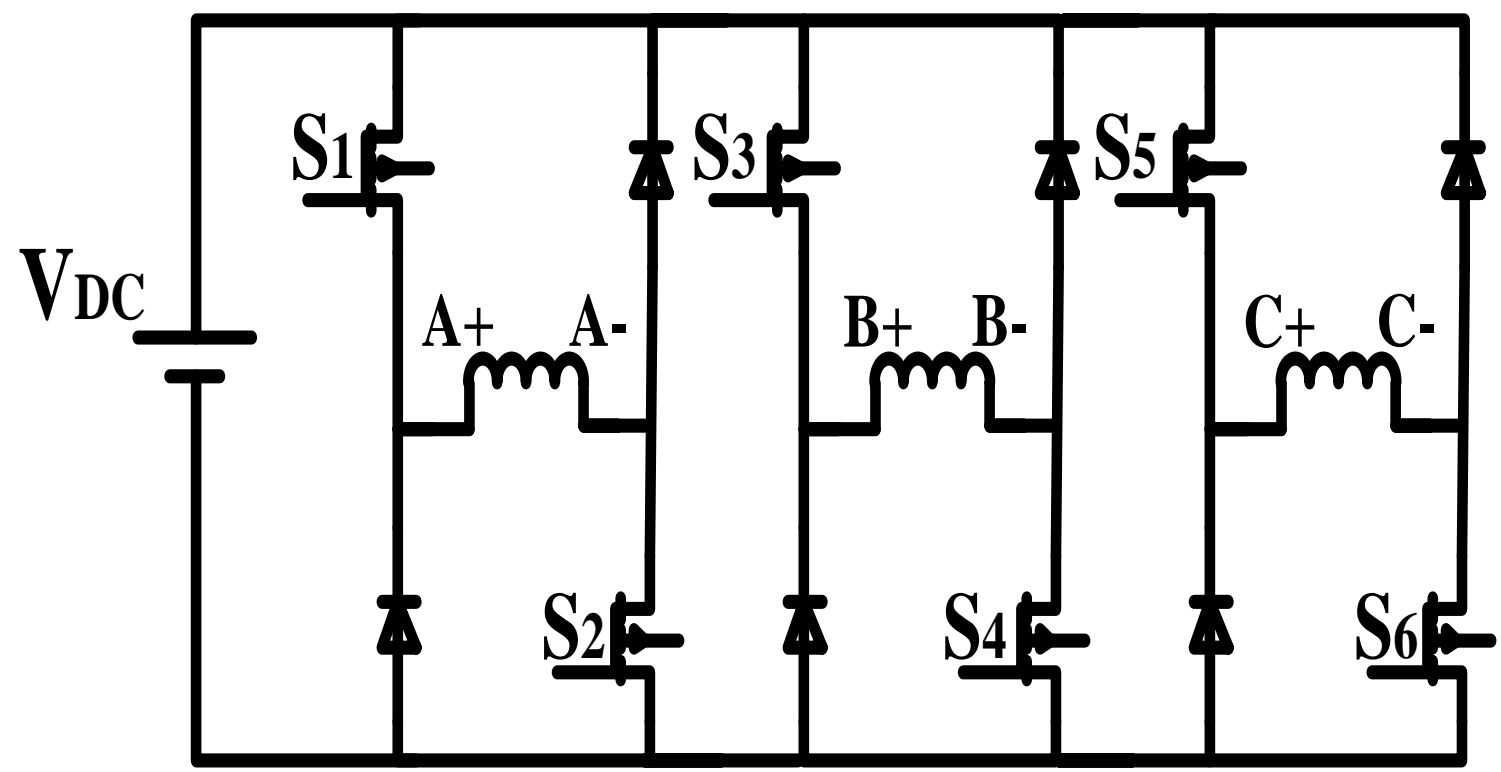

Figure 1.1: SRM asymmetric converter.

The torque production of SRMs at high speeds can be improved by applying a high demagnetization voltage to enable a faster reduction rate to zero the phase current prior to reaching the aligned position [8], before entering the negative torque region. According to [8], the faster demagnetization allows the turn-off angle for the current to be moved closer to the aligned position, which leads to a longer positive torque production region and a lower braking torque. In [2], the authors state that in high speed regions the turn-on angle can be regulated to increase the positive torque production region. However, this also increases the negative torque leading to a lower net torque and efficiency.

Therefore, to extend the speed range of SRMs, there is a need for a method that allows fast demagnetization for the phase excitation currents [8]. Further, it is preferable to develop such a method without the need for additional hardware components in the SRM converters. 
The main concept presented in this thesis is the treatment of each SRM phase winding as a transformer, as each produces a flux in the stator and the rotor magnetic cores. The winding of every phase can then be treated as an autotransformer where a different number of turns could be exposed to the DC bus voltage during magnetization and demagnetization. This configuration achieves a higher volt/turn ratio during demagnetization, thereby enabling a faster demagnetization.

\subsection{Thesis Organization}

The content of the successive chapters in the thesis is briefly described as follows:

Chapter 2 presents SRM theory and operation, stressing the high speed limitations of SRMs. The work developed to improve the high speed attributes of SRMs is also explored in this chapter. Chapter 3 clearly exposes the proposed methodology. The theoretical background, supported by the associated mathematical and circuit analyses, is also covered.

In Chapter 4, the simulation models constructed to verify the proposed concepts are developed and explained. The simulation results are also discussed. Chapter 5 presents a detailed description of the hardware development to implement the proposed winding configuration, including modifications to the conventional machine and the power converter design. Experimental results are also presented and analyzed in this chapter. Chapter 6 elaborates on the conclusions from the thesis work and poses some issues for further study. 


\section{CHAPTER II}

\section{BACKGROUND AND RELATED WORK}

\subsection{Introduction}

SRMs started to attract considerable attention in the early 1980's [9]. As a result, the machine design and its associated drive have been intensely developed since then [10]. SRMs have superior advantages over other electric machines because of their simple structure and high power and torque density. Even so, they have had a limited impact in the automotive industry due mainly to their high torque ripple [11], especially in the high speed region. The work presented in this thesis aims to address these machine limitations, in an effort to improve their high speed operation. This chapter provides a review of SRM operation and control, including previous research related to their high speed control.

\subsection{SRM Operation and Characteristics}

A reluctance motor can be defined as an electric motor in which the electromagnetic torque is developed by the tendency of the moving part to move towards the position where the inductance of the excited phase is maximum while the reluctance of the magnetic path is minimum. This tendency comes from the fact that the flux is always looking for the magnetic path with the minimum possible reluctance. This definition applies to both the SRM (switched reluctance motor) and the synchronous reluctance motor [9] [12]. 
The basic structure of an SRM is depicted in Fig. 2.1, for a motor with 8 stator poles and 6 rotor poles. The SRM is a doubly salient machine; that is, both the stator and the rotor have salient poles. The electrical windings that represent the electromagnetic element are placed on the stator side [13], leaving the rotor poles without a magnet or electrical windings [14] [15]. Further, the windings on the stator poles are connected in series, as shown in Fig. 2.1, for the same phase, although other winding connections can be used [1].

The electrical current driven into the SRM will induce a magnetic flux as shown in Fig. 2.1. The rotor has the freedom to move in order to align itself with the excited

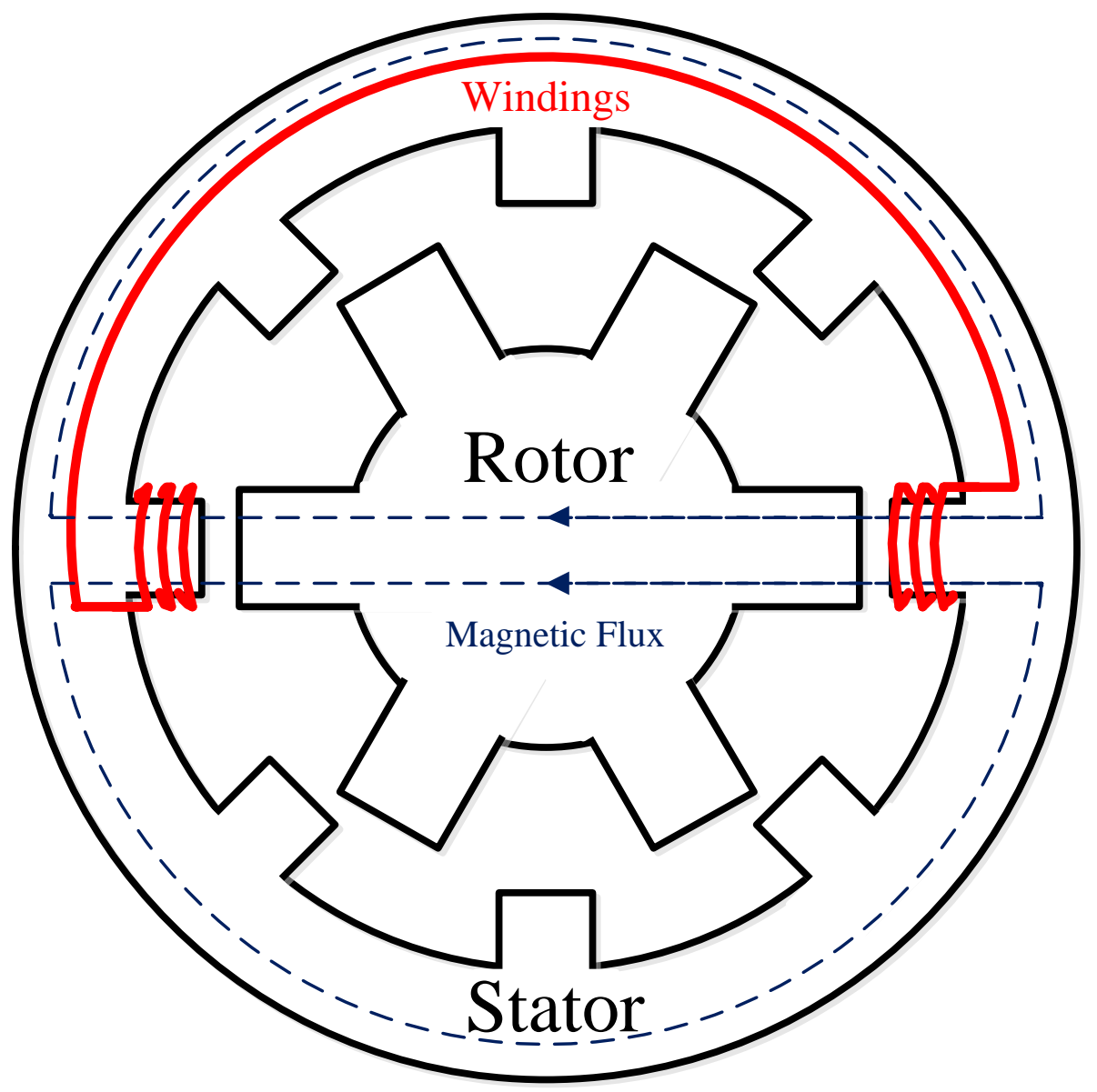

Figure 2.1: SRM basic structure. 
stator pole, with the excited windings. The phase windings should be excited in a sequential manner so as to produce the electromagnetic force required to rotate the motor in the desired direction.

The SRM operation is based on the different reluctances that the flux generated by the excited phase will encounter. This potential difference in the magnetic circuit will force the rotor to move towards the stator with the excited phase; that is, a mechanical force will be exerted on the rotor to align it with the stator pole in a position called the "aligned position," at which point the inductance in the magnetic circuit is at its maximum value [9]. Taking this into consideration, the number of rotor poles is designed to be different from the number of stator poles to avoid the case where the production of the initial torque is not possible (where the rotor poles are aligned with the stator poles) [12].

The inductance profile of SRMs for different rotor positions is an intrinsic parameter that rules the general operation of the motor. The inductance profile of the motor, as seen by one of the phases, is shown in Fig. 2.2. Notice that there are two main regions to be considered:

A. Motor operation: the slope of the inductance change is positive, which represents the appropriate position for the current excitation to operate the SRM in the motoring mode. In this mode, the motor will convert the supplied electrical power into mechanical power.

B. Generator operation: the slope of the inductance is negative, and so the phase excitation in this region will result in a negative (braking) torque production, which is basically the operation of the SRM as a generator. In 
this mode, the motor will convert the mechanical power exerted on the rotor into electrical power, which is provided at the phase's terminals.

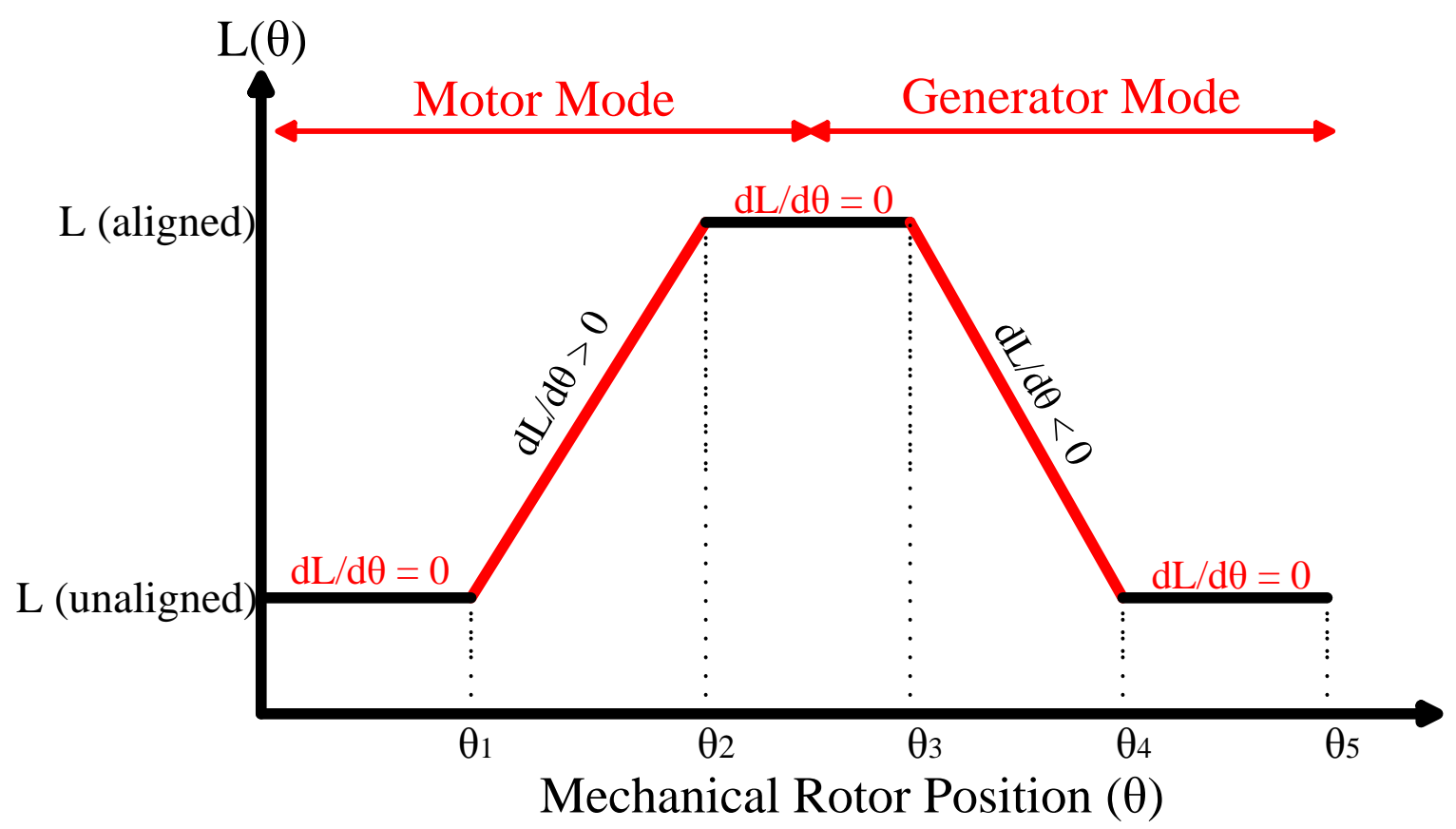

Figure 2.2: Inductance profile for one SRM phase.

The phase inductance will start to increase with the overlapping between the stator and the rotor poles [10], with the maximum inductance being observed at the aligned position where maximum overlapping takes place. From Fig. 2.2 it can also be clearly seen that the minimum inductance is obtained at the unaligned position.

This behavior of the phase inductance is very important for SRM control as it determines the required sequence for the phase excitation in order to sustain the SRM operation, in either the motor or generator mode, in synchronism with the rotor position [16], as will be illustrated later in this chapter. Exciting the phase windings at positions with a constant inductance will produce no torque.

In order to precede with the control analysis a brief model of the SRM and its operation are presented in the following section. 


\subsection{SRM Model}

The SRM model can be described analytically using three linear differential equations, as stated in [12].

A. Electrical dynamics: An equivalent circuit model for an SRM phase winding is shown in Fig. 2.3. The differential equation modeling this circuit is given in Eqn. 2.1, where $\mathrm{V}$ is the voltage applied to the phase winding, $i$ is the phase current, $\mathrm{R}$ is the phase resistance, and $\lambda(\theta, i)$ is the magnetic flux linkage as a function of the current and the rotor position [17]. Notice that Eqn. 2.1 embodies the implementation of Faraday's law [18], considering a weak coupling between the phases [19] :

$$
V=R . i+\frac{d \lambda(\theta, i)}{d t}
$$

The flux linkage $\lambda(\theta, i)$ can be represented by Eqn. 2.2 [12], where $L$ is the phase inductance as a function of the current and the rotor position:

$$
\lambda(\theta, i)=L(\theta, i) i
$$

The flux linkage dependency on the rotor position and the phase current is due to the

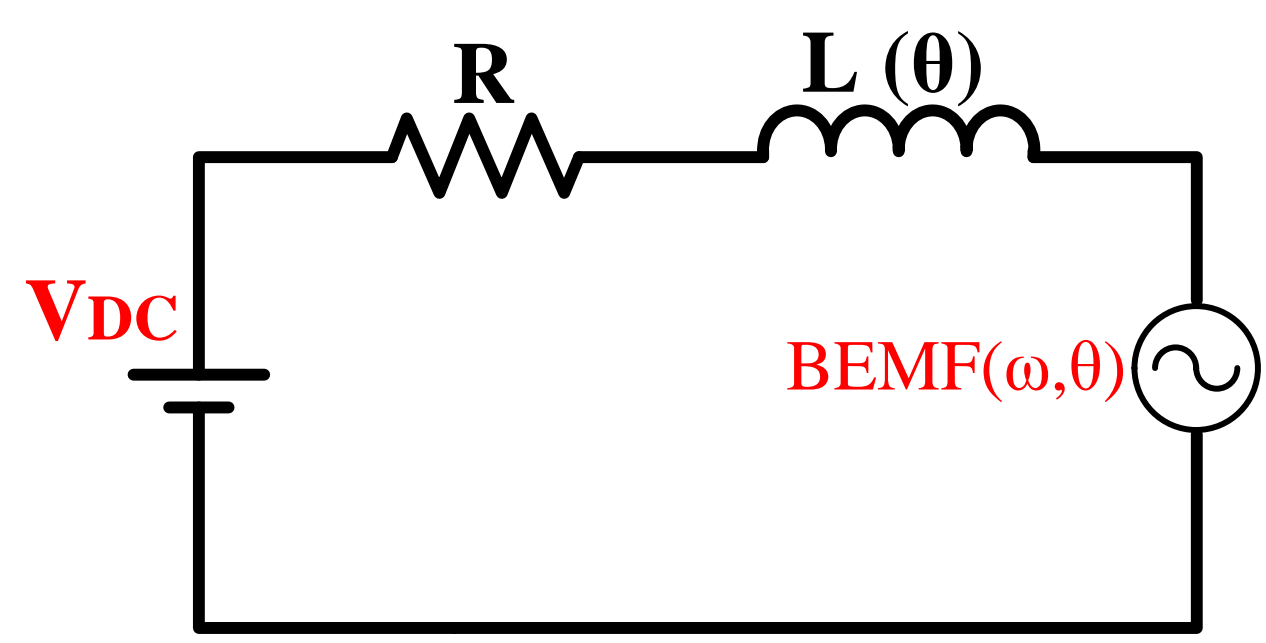

Figure 2.3: SRM phase equivalent circuit model. 
double salient construction of SRMs and the magnetic saturation effects [20].

B. Motional equation: This equation, which describes the phase voltages considering the mechanical rotation of the rotor, describes the transfer of the electrical energy into magnetic energy in SRMs. The term $\frac{d \lambda(\theta, i)}{d t}$ in Eqn. 2.1 represents the back-EMF (electromagnetic force-BEMF). Using Eqn. 2.2 the phase voltage can be described by Eqn. 2.3 [21]. This will be of high importance for the analysis conducted throughout the thesis:

$$
V=R i+\frac{\partial \lambda(\theta, i)}{\partial i} \frac{d i}{d t}+\frac{\partial \lambda(\theta, i)}{\partial \theta} \frac{d \theta}{d t}
$$

Where $\frac{\partial \lambda(\theta, i)}{\partial i}$ represents the instantaneous inductance $L(\theta, i)$ and $\frac{\partial \lambda(\theta, i)}{\partial \theta}$ is the instantaneous back-EMF.

C. Electromagnetic torque equation: This equation describes the conversion from the magnetic energy to the mechanical energy [18]. The electromagnetic torque can be estimated as follows. First, multiply both sides Eqn. 2.3 by the current $i$, to yield:

$$
V i=i^{2} R_{m}+i \frac{d \lambda(\theta, i)}{d t}
$$

Eqn. 2.4 represents the instantaneous electrical power. The first term describes the electrical losses $i^{2} R_{m}$ and, from the conservation of energy, the second term describes both the mechanical power and the magnetic energy stored in the SRM [4]; that is, the rate of change of the mechanical energy and the magnetic field energy, respectively [18]. Hence, the second term in Eqn. 2.4 may be rewritten as:

$$
i \frac{d \lambda(\theta, i)}{d t}=\frac{d W_{m}}{d t}+\frac{d W_{f}}{d t}
$$


Where, $W_{m}$ is the mechanical energy and $W_{f}$ is the magnetic field energy. The mechanical power can be defined as [22]:

$$
\frac{d W_{m}}{d t}=\frac{d W_{m}}{d \theta} \frac{d \theta}{d t}=T \omega=T \frac{d \theta}{d t}
$$

Resulting in a torque equation:

$$
T=\frac{d W_{m}}{d \theta}
$$

where, T represents the electromagnetic torque and $\omega=\frac{d \theta}{d t}$ is the mechanical speed of the motor.

For a simplified case in which the machine operates in the linear region, and the inductance is no longer a function of the current, the electromagnetic torque can be derived as follows [18]:

$$
\begin{gathered}
\lambda(\theta)=L(\theta) i \\
W_{m}=\frac{i^{2}}{2} L(\theta) \\
T=\frac{d W_{m}}{d \theta}=\frac{1}{2} i^{2} \frac{d L}{d \theta}
\end{gathered}
$$

Eqn. 2.10 represents the most familiar mathematical representation for the electromagnetic torque, and which clearly clarifies the two components responsible for developing the electromagnetic torque; namely, the electric current and the rate of change of phase's inductance.

For further analysis, SRM torque equations can be derived using the energy and co-energy concepts that consider the magnetic saturation, which is beyond the scope of the thesis. 


\subsection{SRM Control}

SRM phases should be excited in a sequential manner in order to maintain torque production in either motoring or generating mode in synchronism with the rotor position [4] [23]. The SRM drive should be able to measure or estimate the rotor position and accordingly excite the corresponding phase for the required motor operation.

The phase excitation is defined by three control parameters which are turn-on angle, turn-off angle, and the current reference [16]. The turn-on angle is the electrical or mechanical angle for the motor rotation at which the controller starts the phase's excitation. The turn-off angle is the angle at which the excitation is terminated. The turnon and turn-off angles are usually determined according to the machine inherent characteristics (inductance profile). However, different control objectives like torque regulation and current limitation are also considered for turn-on and turn-off angle optimization. For a smooth and uniform SRM operation, the same control parameters are applied to all of the machine phases [16].The phase's current reference is dictated by the thermal limitation of the machine as well as the torque and speed control requirements.

The SRM drive consists mainly of a power converter, a position detector, and a controller [24], in addition to the current sensors for current regulation purposes. The converter topology and its control are of high importance for the machine total efficiency; this correlation justifies ongoing research aimed improved converter performance in order to enhance SRM operation.

In [25] the authors summarized the basic requirements for SRM drives to be as follows:

- Phase voltage modulation to achieve phase current control. 
- Maximum possible voltage gain to operate the machine at high speeds.

- The minimum possible demagnetization time; which is the main objective of the work proposed in this thesis.

- Full control of current excitation and demagnetization for different phases simultaneously to reduce the torque ripple.

- The converter should be universal and independent of the machine construction.

- The minimum possible number of semiconductors needed should be obtained; which is another important consideration of the work proposed in the thesis.

In [26] the authors prepared a comparative evaluation for the inverter power circuits used to run SRMs, all of which are designed to meet the above mentioned requirements. According to [7] the most common power converter used for SRMs is the asymmetric converter shown in Fig. 1.1 [7].

The converter consists of two switches and two diodes per phase. The two switches are responsible for controlling the driving current for each phase, while the two diodes are required to provide a path for free-wheeling and for the discharge current to be pushed back to the converter DC bus [7]. The work presented in this thesis will focus only on the asymmetric power converter.

According to [27], the operational modes of the SRM converter, for one phase excitation, can be divided into 3 different modes. As following:

A. Magnetization or charging mode: In this mode the DC bus voltage is applied across the phase windings, allowing the phase current to freely build 
up within a certain limit defined by the controller. For the magnetization mode, the two switches are $\mathrm{ON}$ while the two diodes are reversed biased as shown in Fig. 2.4.

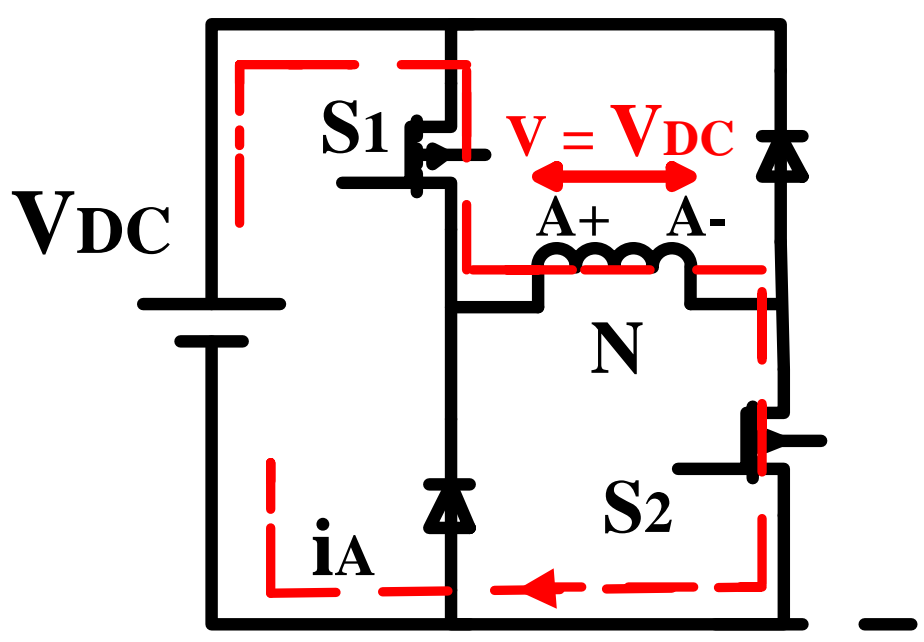

Figure 2.4: SRM magnetization mode for the asymmetric converter.

B. Freewheeling mode: This is a current control mode in which zero voltage is applied across the phase windings, to allow the current to discharge slowly in order to follow the desired reference current defined by the controller.

For the freewheeling mode, one of the switches and its associate diode are ON and thus the current will flow as shown in Fig. 2.5. 


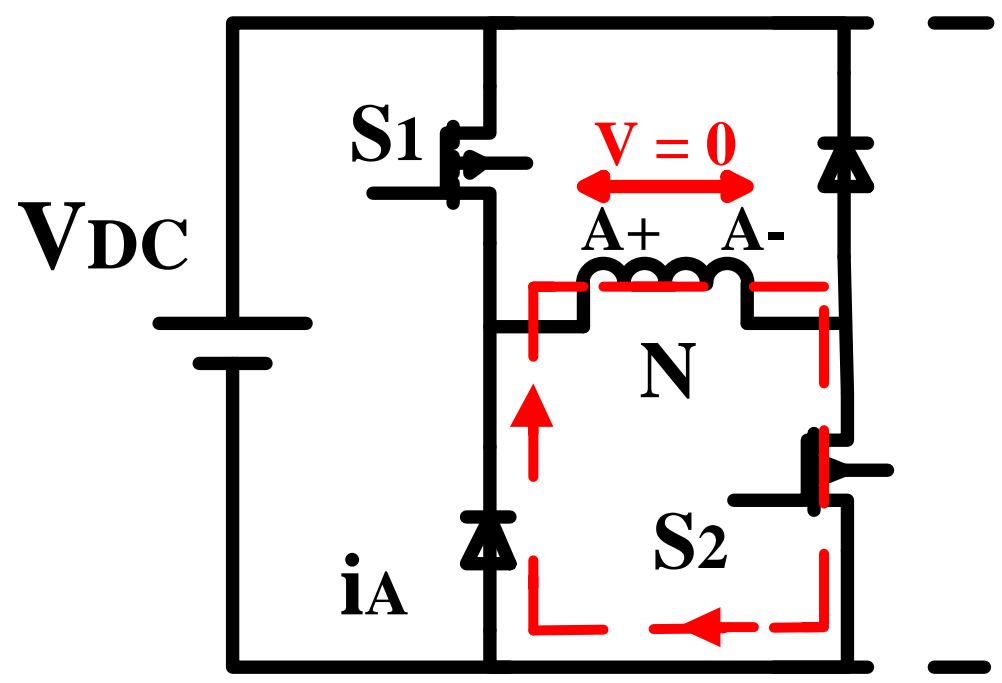

Figure 2.5: SRM freewheeling mode for the asymmetric converter.

C. Demagnetization mode: This is the important mode for the work proposed in this thesis. The demagnetization starts when the controller turns the two switches OFF, and the phase windings will be connected to the DC bus with a reversed configuration, hence a negative DC bus voltage will be applied across the phase winding through the two diodes. The two diodes will be forward biased as shown in Fig. 2.6 due to the residual current in the coils [10]. The main control objective behind this mode is to quickly discharge the phase current before the rotor leaves the aligned position; that is, before getting into the negative torque production region. 


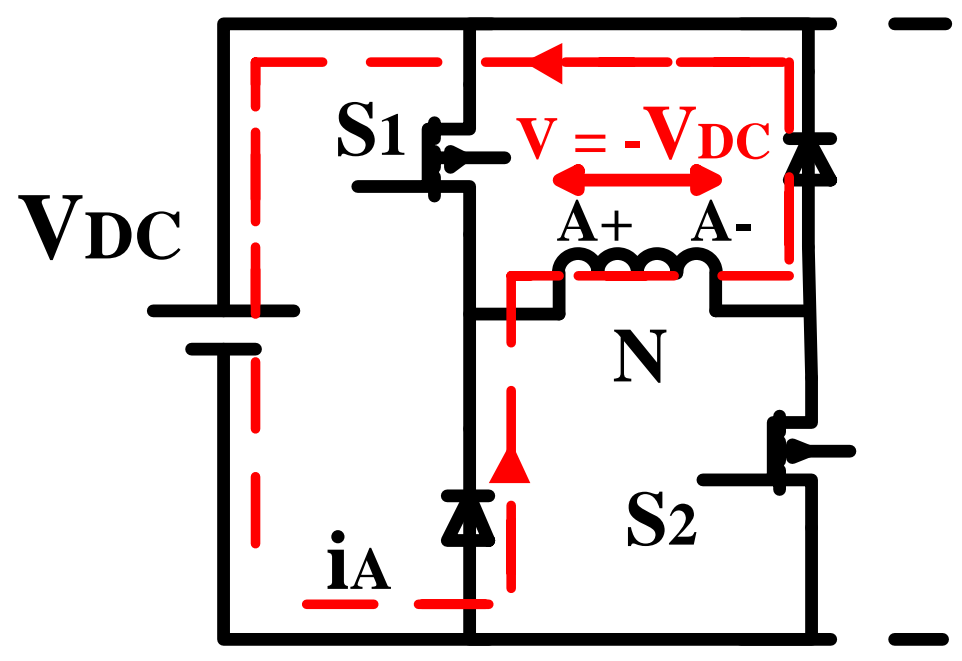

Figure 2.6: SRM demagnetization mode for asymmetric converter.

\subsection{SRM Speed Regions}

It is always desirable to extract the maximum possible power out of electric machines, though there are different considerations to be taken into account while running the machine at different operating conditions.

The torque-speed curve is a very important descriptive plot that can characterize the machine performance at different operating conditions; it illustrates the relationship between the motor speed and the maximum torque that the machine can produce at that speed [28] as shown in Fig. 2.7. It also describes the maximum power that the machine can produce. Since the output of any electric machine is defined by the equation:

$$
P_{\text {out }}=T \omega
$$




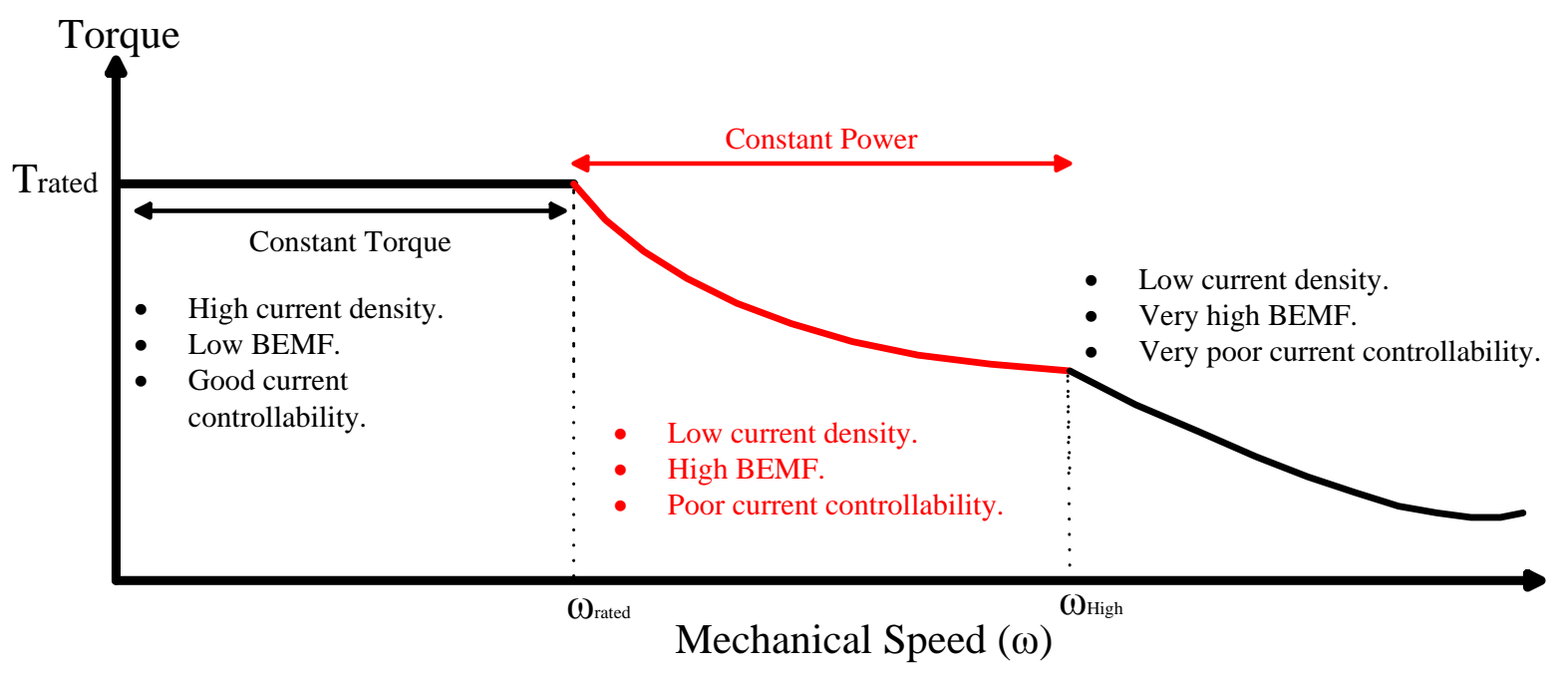

Figure 2.7: SRM torque-speed curve.

where $P_{\text {out }}$ is the output power produced by the machine, $T$ is the mechanical torque, and $\omega$ is the rotor speed. The machine operation has different restrictions and limitations for different speed loadings. At low speeds the torque production is limited by the maximum current that the machine can tolerate considering its thermal limitations. For high speeds there are other restrictions, such as the high back EMF voltage which significantly limits the current building with the increases in the motor speed, as seen from Eqn. 2.3.

The other limitation is the short time available for the current to build and discharge at high speeds. Intuition indicates that for a synchronous machine, like an SRM, the electrical cycle decreases as the motor speed increases.

The short electrical cycle typically results in a tail current that extends to the negative torque region and hence reduces the net torque produced by the machine. The main focus of this thesis is to overcome the current discharge problem at high speeds.

Improving the current discharge rate can result in a higher positive torque, as the magnetization time can be extended (higher turn-off), and also lower negative torque 
production as the current will be set to zero before reaching the negative torque regions [8].

\subsection{Related Work}

Improving the demagnetization mode is an ongoing research topic for SRM drives. In [8] a new power converter that can work as a boost converter during the demagnetization to improve SRM high speed operation is presented. Separate windings were used for magnetization and demagnetization for the purpose of realizing a simple hardware structure in the converter [7]. However, the two windings have the same number of turns and as such they provide no enhancement in the SRM high speed performance.

The current discharge rate can be increased by imposing a high negative voltage during the demagnetization mode [8], compared to the voltage applied to the phase windings during the magnetization mode. Hence a better torque and power production can be achieved; as higher current levels can be obtained with higher discharge rate for the current.

The authors in [29] are proposing to use a four-level converter by which a higher voltage can be applied to speed up both the magnetization and demagnetization modes and hence improve torque production at high speeds.

These driver designs will add more complexity to the control as well as an additional cost for the driver. It also requires a higher DC bus voltage for the multi-level converter to control different voltage levels at different motor speeds. 
A complicated driver and a winding configuration, with three leads for each phase, are introduced in [30]. The driver includes extra switches that can connect the two windings in series to obtain the typical operation of the asymmetric converter at low speeds, while at high speeds the two coils can be connected in parallel in order to apply a high voltage across each winding and hence improve the machine performance.

This approach suffers from the disadvantages of the work in $[8,29]$, beside the possibility of introducing an unbalanced magnetic pull force when connecting the two windings in parallel. According to [31], by adding an extra coupled inductor per phase, which is dedicated to discharging the phase current during the demagnetization, demagnetization can be improved.

The coupled inductor has two windings, $L_{1}$ and $L_{2}$, which are connected in a transformer configuration, while one of them is connected in series with the phase winding as shown in Fig. 2.8.

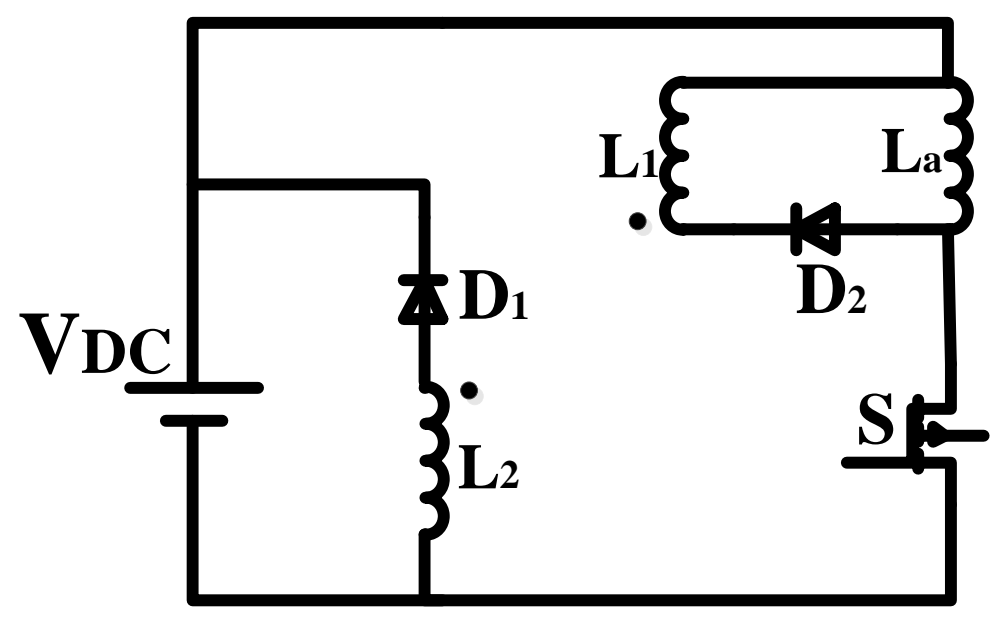

Figure 2.8: Converter topology for fast demagnetization.

The demagnetization voltage can be increased by adjusting the coupled inductor turns ratio. This approach is very similar to the work presented in this thesis. While it 
has the advantage of a limited number of components and simple control, additional windings should be considered for each phase with the proper magnetic coupling between them.

\subsection{Conclusion}

The definition, operation, and control of SRMs were introduced in this chapter. The high speed operation of SRMs was highlighted to disclose the motivation of this thesis. The slow current discharge rate of SRMs at high speeds is the main limitation to be addressed throughout the thesis. A brief review of related work to enhance the demagnetization capabilities of SRMs was covered. The next chapter proposes novel concepts to improve the demagnetization mode of SRMs. 


\section{CHAPTER III}

\section{THE PROPOSED THESIS WORK}

\subsection{Introduction}

The main concept of the thesis work is to split the stator phase winding into two portions; namely, the primary and the secondary windings, to provide better phase current control. The voltage applied to the main winding during demagnetization is boosted by the new winding topology, leading to a higher discharge rate of the current and hence better efficiency at high speeds. The phase inductances seen by the DC bus will decrease as a result of eliminating a portion of the winding from the demagnetization process.

\subsection{Split Phase Winding Scheme}

The proposed split phase winding structure can be considered as an autotransformer connection scheme. The topology can be implemented by interconnecting the stacked primary and secondary windings as shown in Fig. 3.1. The magnetic coupling between the two windings defines the transformer operation between them. Theoretically, the leakage inductance for the two windings should be close to zero. For the new configuration to be introduced in an off-the-shelf SRM a rewinding is required, while the machine geometrical and physical parameters will remain the same. 


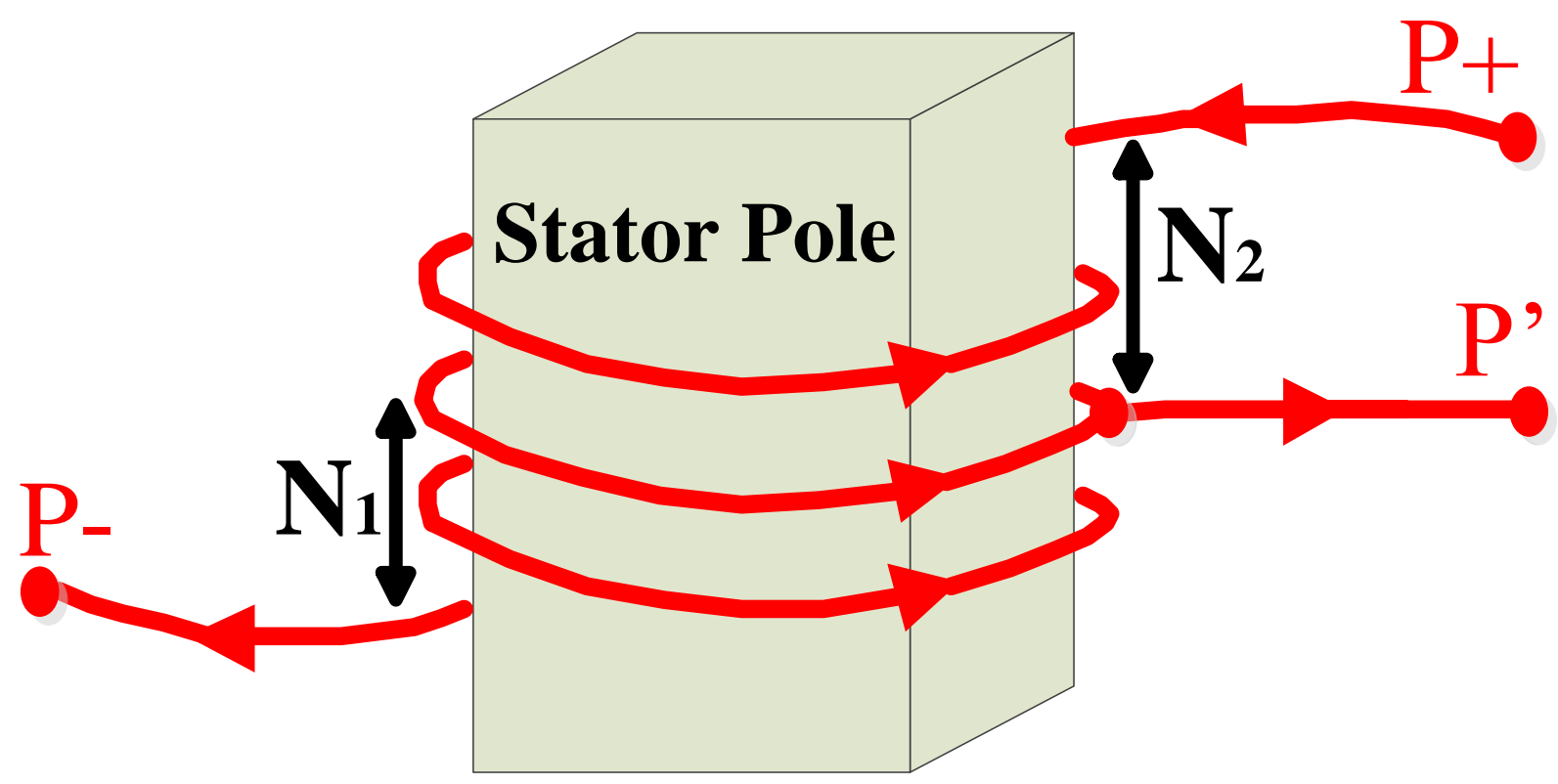

Figure 3.1: Autotransformer winding configuration in the stator pole.

The number of turns of the conventional winding structure is restructured into two separate windings. One of the windings is designed to have a higher number of turns to ensure a higher gain for the demagnetization voltage. However, further considerations for the voltage and the current limitations of the power converter components should be taken into account. The optimization of a number of turns for the two portions of the winding is an important factor to utilize the machine with the new winding configuration. The higher voltage per turn and the lower winding inductance can result in a higher current discharge rate. The power converter design should be adjusted to the new configuration. On the one hand, no extra power electronics components are needed to drive the motor with the new winding topology. On the other hand, the components need to be connected in a different way to allow the current flow in the desired way.

The control algorithm for the new SRM structure circuit, in terms of components and control algorithm will not change except for the current regulation. The hard 
chopping for the hysteresis current control cannot be implemented in the SRM with the new winding configuration as will be explained in the later chapters of the thesis.

\subsection{Proposed Power Converter}

The power converter associated with the new winding topology is shown in Fig. 3.2. The two switches are placed on the two edges of the full phase windings while the two diodes are connected across a portion of that winding. The new converter is similar to the conventional converter in terms of the number of switches and diodes associated with each phase. The converter modes of operation can be summarized as follows:

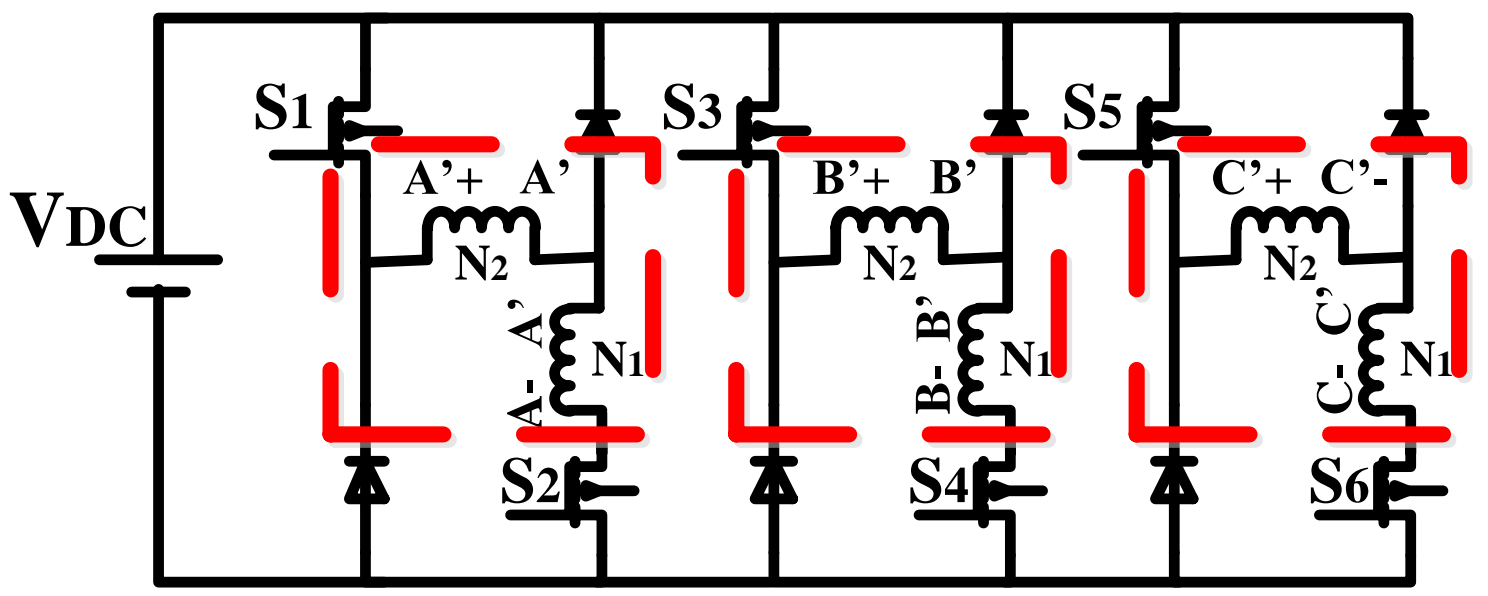

Figure 3.2: The proposed power converter.

A. Magnetization mode: The power converter in the magnetization mode is shown in Fig. 3.3. In this mode the two portions of the winding will be connected in series, the machine operation will be typical to the normal SRM operation in terms of currents, voltages, and torque production. The voltage across the series connected windings is $V_{d c}$ during this mode. 


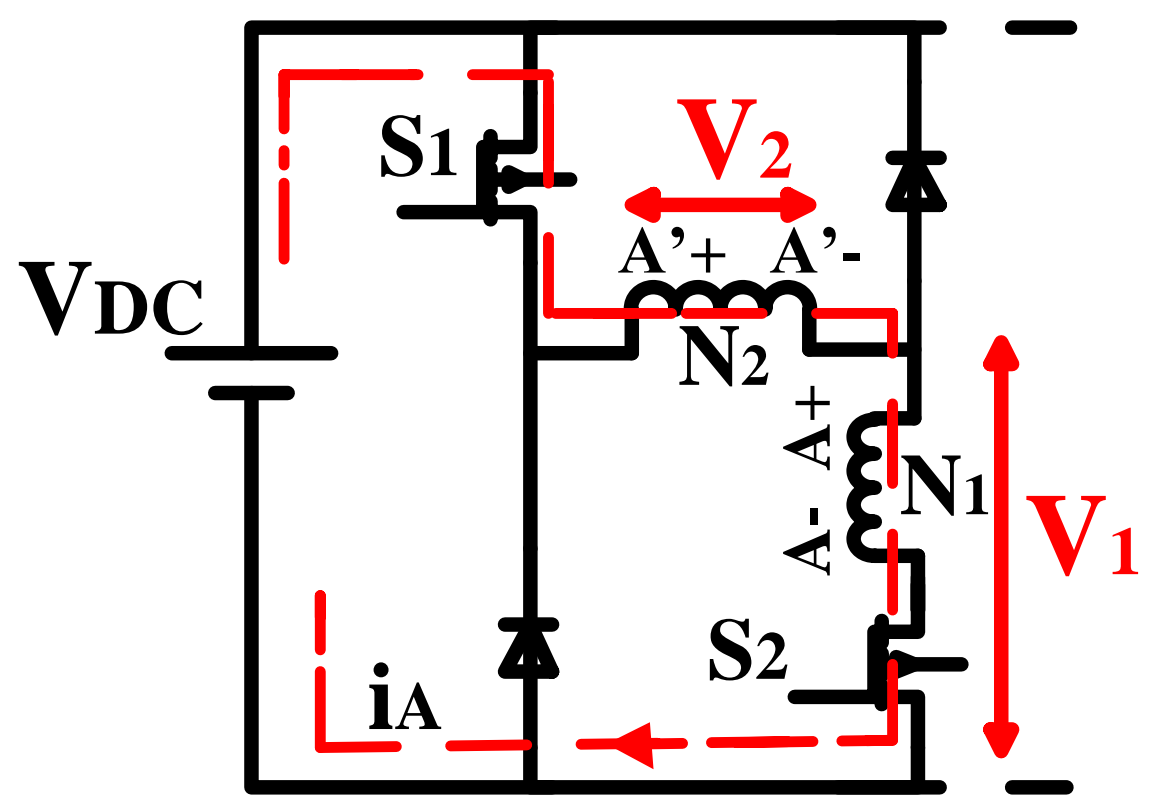

Figure 3.3: Energizing the main windings.

B. Freewheeling mode: The power converter in the freewheeling mode is shown in Fig. 3.4. The primary $\left(N_{1}\right)$ and the secondary $\left(N_{2}\right)$ windings are connected in series and the total voltage applied to the full winding will be zero. It should be noted that with the new converter freewheeling should be accomplished by turning the upper switch ON and OFF while keeping the lower switch $\mathrm{ON}$ for the full conduction time. The current cannot be regulated by controlling the lower switch because the primary winding $N_{1}$ will be excluded from the current path when turning the lower switch OFF. This is the soft chopping of the current; in which zero voltage will be applied across the phase windings. 


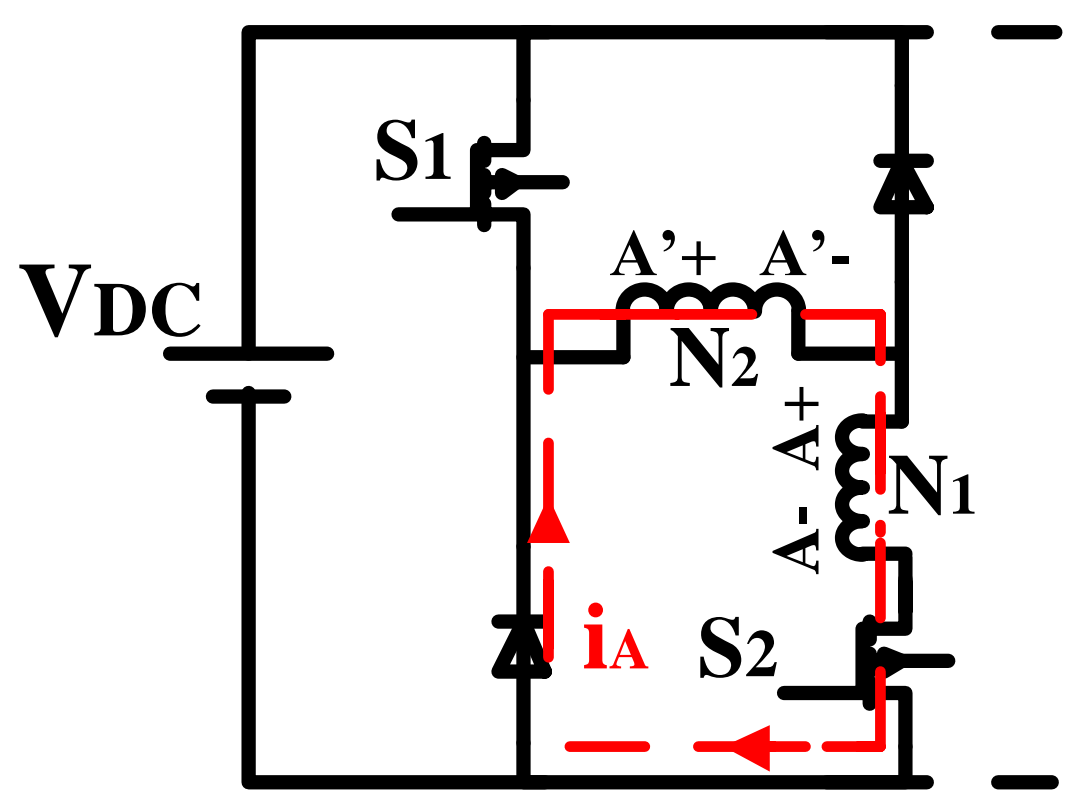

Figure 3.4: Freewheeling mode for the new converter.

The freewheeling operation is similar to that of the conventional drive.

However, the hard chopping control for the current is not applicable with the new drive, as this will also exclude the primary windings and apply the full bus voltage across the secondary windings.

C. Demagnetization mode: This is the mode of interest for the thesis work. The converter configuration for the demagnetization is shown in Fig. 3.5. In this mode the controller will turn both switches OFF, connecting the full DC bus voltage across the secondary winding $N_{2}$ through the two diodes. The current will be instantaneously cut from the primary winding $N_{1}$.

The magnetic coupling between the two portions will link the whole flux to the $N_{2}$ winding. The turns ratio between the two windings dictates the time required to discharge the phase current; that is, the smaller $N_{2}$ is the shorter the demagnetization time will be. 


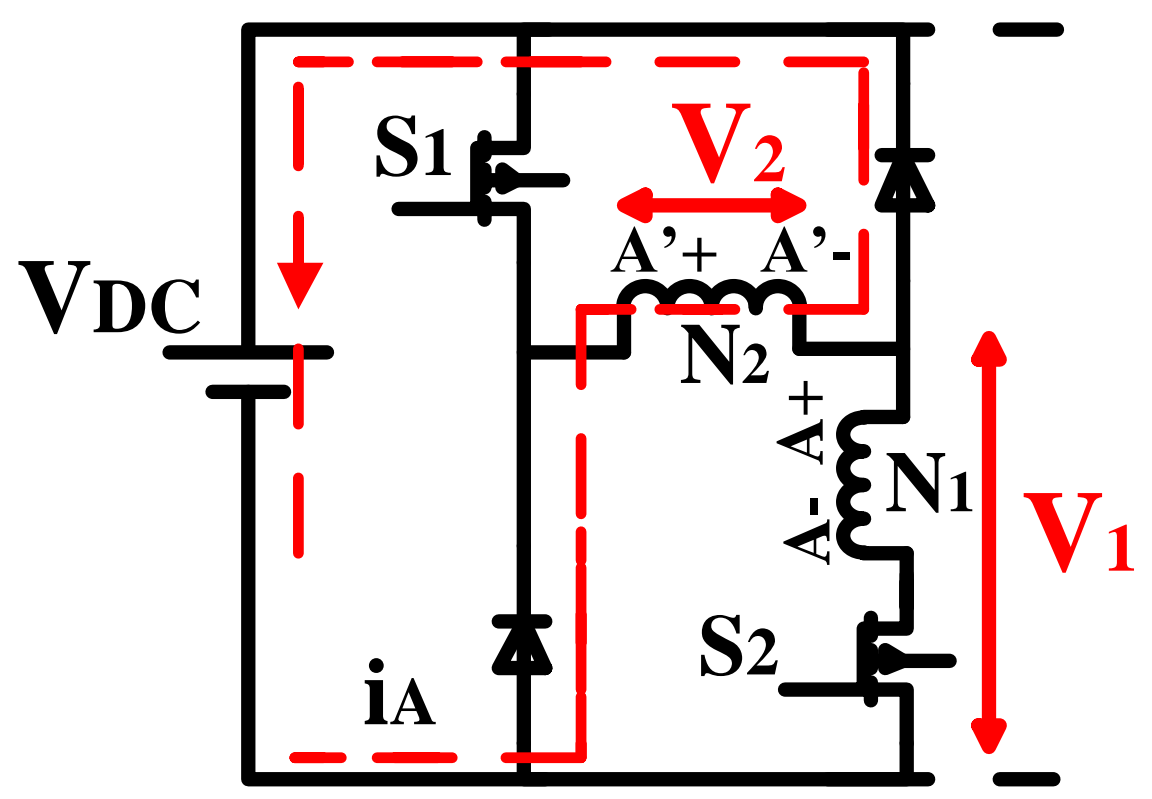

Figure 3.5: Demagnetization through the secondary windings.

\subsection{Analysis of the Converter Operation}

This section provides the mathematical analysis of the new power converter, to verify the improvement in the demagnetization mode. For the power converter, during driving the machine phases with the excitation current, both switches for the phase will be $\mathrm{ON}$ as shown in Fig. 3.3. The voltages across the phase windings can be represented as follows:

$$
\begin{aligned}
& V_{1}+V_{2}=V_{d c} \\
& \frac{V_{1}}{V_{2}}=\frac{N_{1}}{N_{2}} \\
& V_{2}=\frac{N_{2}}{N_{1}+N_{2}} \times V_{d c}
\end{aligned}
$$


which indicates that the voltage across the secondary winding $V_{2}<V_{D c}$. During the demagnetization time both switches are OFF as shown in Fig. 3.5. Where the voltages can be represented as follows:

$$
\begin{aligned}
& V_{2}=V_{d c} \\
& V_{1}=\frac{N_{1}}{N_{2}} V_{d c}
\end{aligned}
$$

Comparing Eqns. 3.3 and 3.4 it can be seen that $V_{2}$ is increased during demagnetization. As the voltage $V_{2}$ increases, faster demagnetization is expected. During the demagnetization the current will be cut completely from the main winding and, since the two coils are wrapped around the same core, the main coil flux will cause the current level in the $\mathrm{N}_{2}$ winding to increase very fast. As a matter of fact, the new level of the current is magnified by $M=\frac{N_{1}+N_{2}}{N_{2}}$.

The jump in the current level according to the transformer turns ratio will be instantaneous as shown in Eqn. 3.6:

$$
\frac{I_{2}}{I_{\text {original }}}=\frac{N_{\text {total }}}{N_{2}}=\frac{N_{1}+N_{2}}{N_{2}} \rightarrow I_{2}=M I_{\text {original }}
$$

where $I_{2}$ is the secondary winding current, $I_{\text {original }}$ is the total phase current during the magnetization mode, $N_{\text {total }}$ is the total number of turns in the primary and secondary combined.

It is clear, after cutting the current from the primary winding, that the current relationship in Eqn. 3.6 is no longer valid. The inductance of $N_{2}$ can be related to the total inductance as follows, 


$$
\begin{gathered}
L_{\text {mag }}=\frac{\mu A\left(N_{1}+N_{2}\right)^{2}}{l} \\
L_{\text {demag }}=\frac{\mu A N_{2}{ }^{2}}{l} \\
L_{\text {demag }}=\left(\frac{N_{2}}{N_{1}+N_{2}}\right)^{2} \times L_{\text {mag }}
\end{gathered}
$$

where $L_{\text {mag }}$ denotes the inductance of the two windings combined during the magnetization time, $L_{\text {demag }}$ denotes the secondary winding inductance during the magnetization time, $\mu$ is the magnetic permeability of the stator pole, $\mathrm{A}$ is the cross sectional area of the magnetic circuit, and $l$ is the length of the magnetic path. From Eqns. 3.6 and 3.9, the current waveform of the secondary winding will encounter an instantaneous jump followed by a fast drop in the current to zero as will be shown in Chapter 4.

To provide a simplified explanation of the improvement in the current discharge rate, consider the operation in the linear region of the $\lambda-i-\theta$ characteristics of SRM, where the inductance is not a function of the phase current. The amount of the phase current to be discharged during the demagnetization for the conventional SRM can be explained as [12]:

$$
-V_{d c}-\frac{d \lambda_{a}}{d \theta} \omega=L_{a, \theta} \frac{d i_{a}}{d t} \approx L_{a, \theta} \frac{\Delta i_{a}}{\Delta t} \rightarrow \Delta i_{a}=-\left(V_{d c}+\frac{d \lambda_{a}}{d \theta} \omega\right) \frac{\Delta t}{L_{a, \theta}}
$$

where $L_{a, \theta}, i_{a}$, and $\lambda_{a}$ are respectively the inductance, the current, and flux linkage of phase A. $\theta$ is the rotor position and $\omega$ is the motor speed. As the motor speed increases, the time $\Delta t$ available to demagnetize the phase current becomes smaller and thus the 
motor must be demagnetized earlier to avoid operating in the negative torque region. On the other hand, in the proposed method, the amount of the phase current can be predicted as:

$$
-V_{d c}-M \frac{d \lambda_{a}}{d \theta} \omega \approx M L_{a, \theta} \frac{d i_{a}}{d t} \rightarrow \Delta i_{a}=-\left(V_{d c}+M \frac{d \lambda_{a}}{d \theta} \omega\right) \frac{\Delta t}{M L_{a, \theta}}
$$

Comparing Eqns. 3.10 and 3.11, it can be seen that the ratio of the demagnetization time in the proposed configuration and the conventional one is less than $1 / M: 1$. Thus, the demagnetization time is considerably improved with the new winding topology.

\subsection{Conclusion}

This chapter presented the proposed work to improve the high speed characteristics of SRMs. The auto-transformer winding configuration is introduced along with the associated power converter design. The demagnetization mode was discussed in detail to clarify the improvement in the current discharge rate. The proposed work was supported by mathematical derivations to verify the significance of the work. 


\section{CHAPTER IV}

\section{SYSTEM MODELLING AND SIMULATION}

\subsection{Introduction}

In this chapter, detailed models of the conventional and the proposed SRM are built. The power converter and its associated control are developed and interfaced to the SRM models to emulate the operating modes of the SRM.

\subsection{SRM Finite Element Model}

The models of the electric motors are usually constructed from the differential equations describing the electrical behavior and the mechanical behavior of the motor while accounting for the coupling between the two models. However, for a detailed model that considers the geometrical parameters, besides the variation of different electrical parameters with respect to saturation, overlapping between the poles, material properties, and operating conditions of the machine a detailed finite element analysis (FEA) is required [9] [1]. FEA is capable of emulating the real case scenario of the electric motor under study. A detailed description of the electrical and mechanical parameters of the motor is required to build the finite element model. The power converter and its control algorithm should be interfaced with the model to simulate the motor operation. For the analysis derived in this thesis 2D (two-dimensional) models of the SRM are developed to simulate both the conventional and the new (proposed) motor. The software package FLUX 12.1 by CEDRAT is used to build the models. 


\subsubsection{Conventional Machine Model}

The 2D model defines the motor parts including the shaft, the rotor, the air gap, the phase windings, the stator tooth, and the back iron of the stator. The FE model for the conventional motor is shown in Fig. 4.1. The detailed description of the mechanical and electrical parameters of the model is depicted in Table 4.1. The FE model is built according to a case study SRM used for washing machine application, as will be discussed in Chapter 5. The motor is a 12/8, $100 \mathrm{~W}$ motor with a corner speed of 1000 rpm. The simulation is performed by moving the rotor for one mechanical degree and estimating the electrical and the mechanical variables in terms of the voltages, currents, flux-linkage, mechanical speed, the and electromagnetic torque.

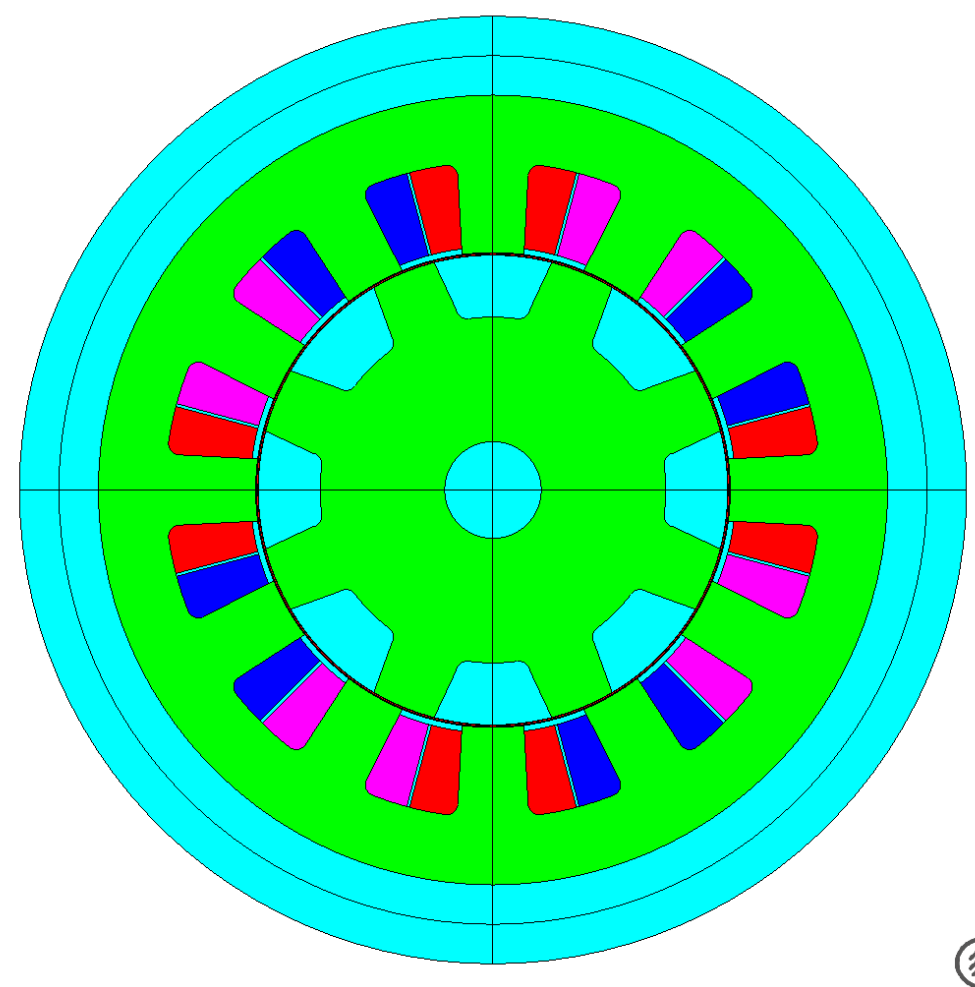

Figure 4.1: Conventional SRM motor finite element model. 
Table 4.1: Finite element SRM model parameters

\begin{tabular}{cc}
\hline Parameter & Value \\
\hline Rotor outer radius & $41.49 \mathrm{~mm}$ \\
\hline Rotor internal radius & $30.5 \mathrm{~mm}$ \\
\hline Machine outer radius & $69.76 \mathrm{~mm}$ \\
\hline Shaft radius & $8.54 \mathrm{~mm}$ \\
\hline Number of stator poles & 12 \\
\hline Number of rotor poles & 8 \\
\hline Stack length & $46.49 \mathrm{~mm}$ \\
\hline Number of phases & $3 \mathrm{phases}$ \\
\hline Number of turns & $2 \mathrm{paths}$ \\
\hline
\end{tabular}

\subsubsection{The Proposed Motor Model}

The new winding topology is introduced on the FE model of the conventional model by dividing the stator slot according to the turns ratio between the primary and the secondary windings as shown in Fig. 4.2. Two separate windings are placed in these slots and connected in series to emulate the new split-winding concept. The total slot area for the conventional machine model is $104 \mathrm{~mm}^{2}$. This area is divided according to the ratio of the primary to the secondary winding number of turns; that is, for $N_{1}=100$ turns and $N_{2}=50$ turns. The inductance profile of the proposed motor model is shown in Fig. 4.3. 


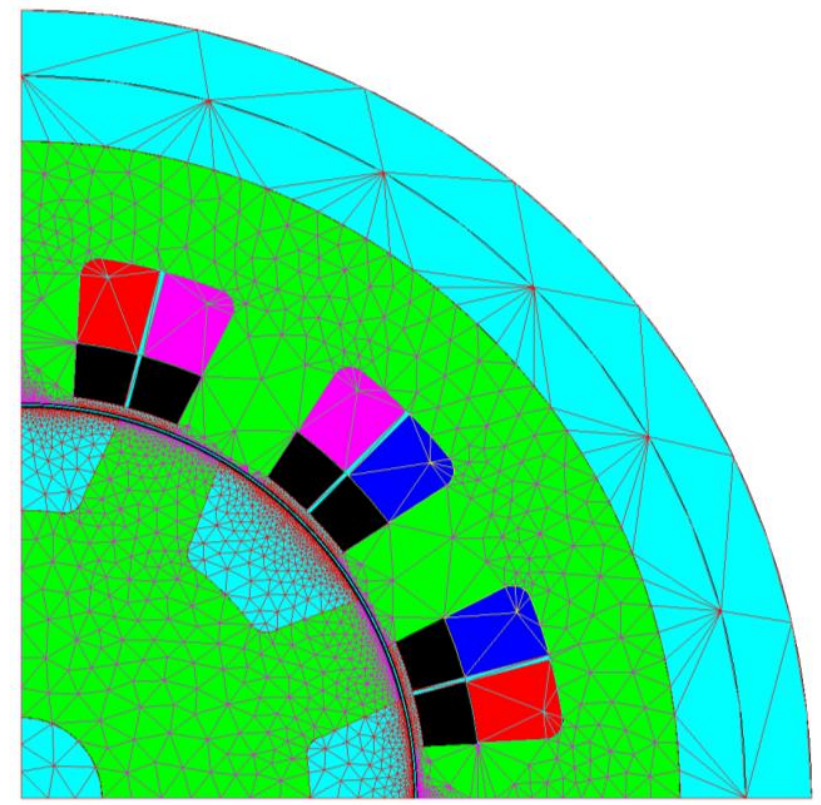

Figure 4.2: The new motor finite element model.

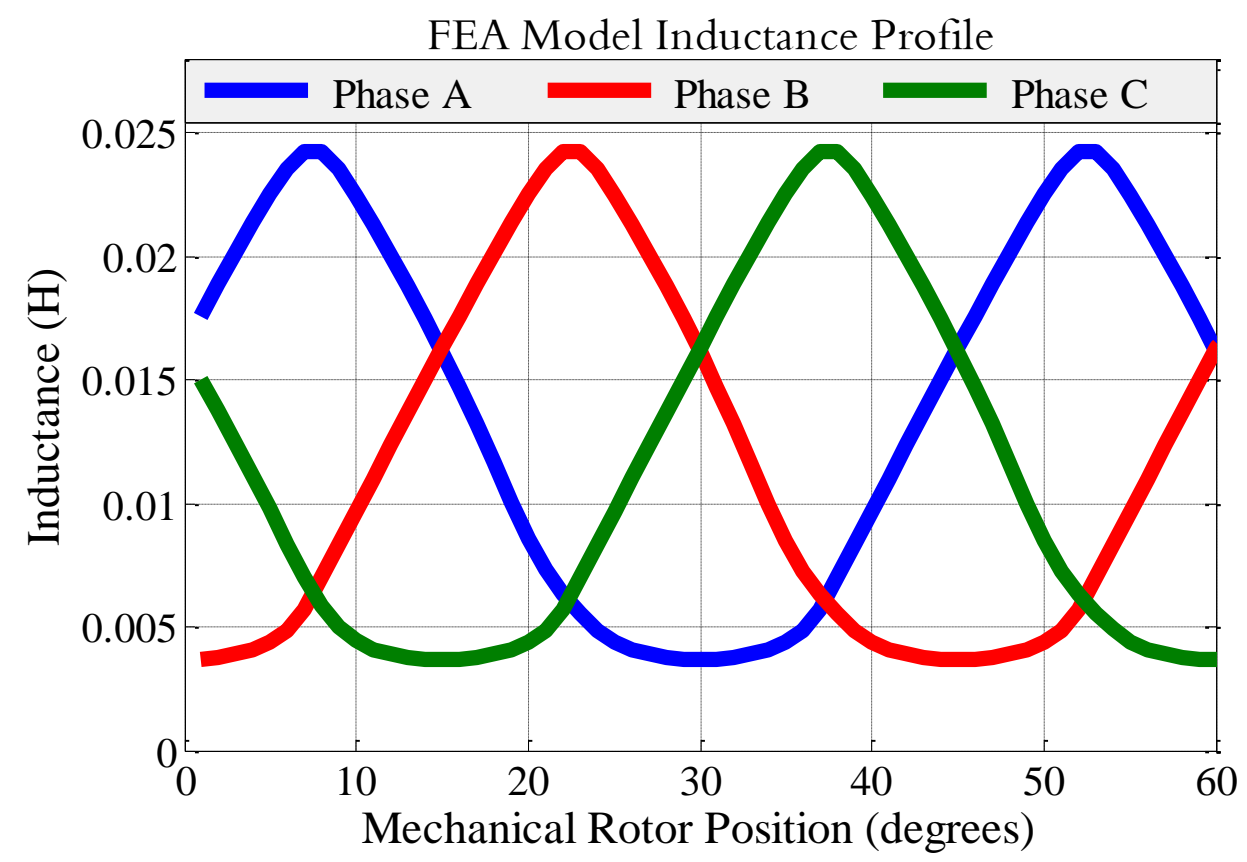

Figure 4.3: The inductance profile of the new motor model.

\subsection{Power Converter Model}

The FE model is interfaced to a power converter designed in the circuit editor of

Flux 12.1. The power converter for the conventional motor, shown in Fig. 4.4, consists of two diodes and two switches per phase. The power electronic switches are modeled 
with a switch that has a resistance of $0.1 \mathrm{~m} \Omega$ for the ON state and $10 \mathrm{k} \Omega$ for the OFF state. Similarly, the diodes are also modeled to have a $0.1 \mathrm{~m} \Omega$ in the forward bias state and $10 \Omega$ resistance in the reverse bias state. The phase windings in Fig. 4.4 are placed in the stator slots of the FE model of Fig 4.2. Each winding in the converter model corresponds to two windings in parallel in the FE model. The power converter switching commands are generated based on the rotor position feedback to track the positive torque production region for each phase.

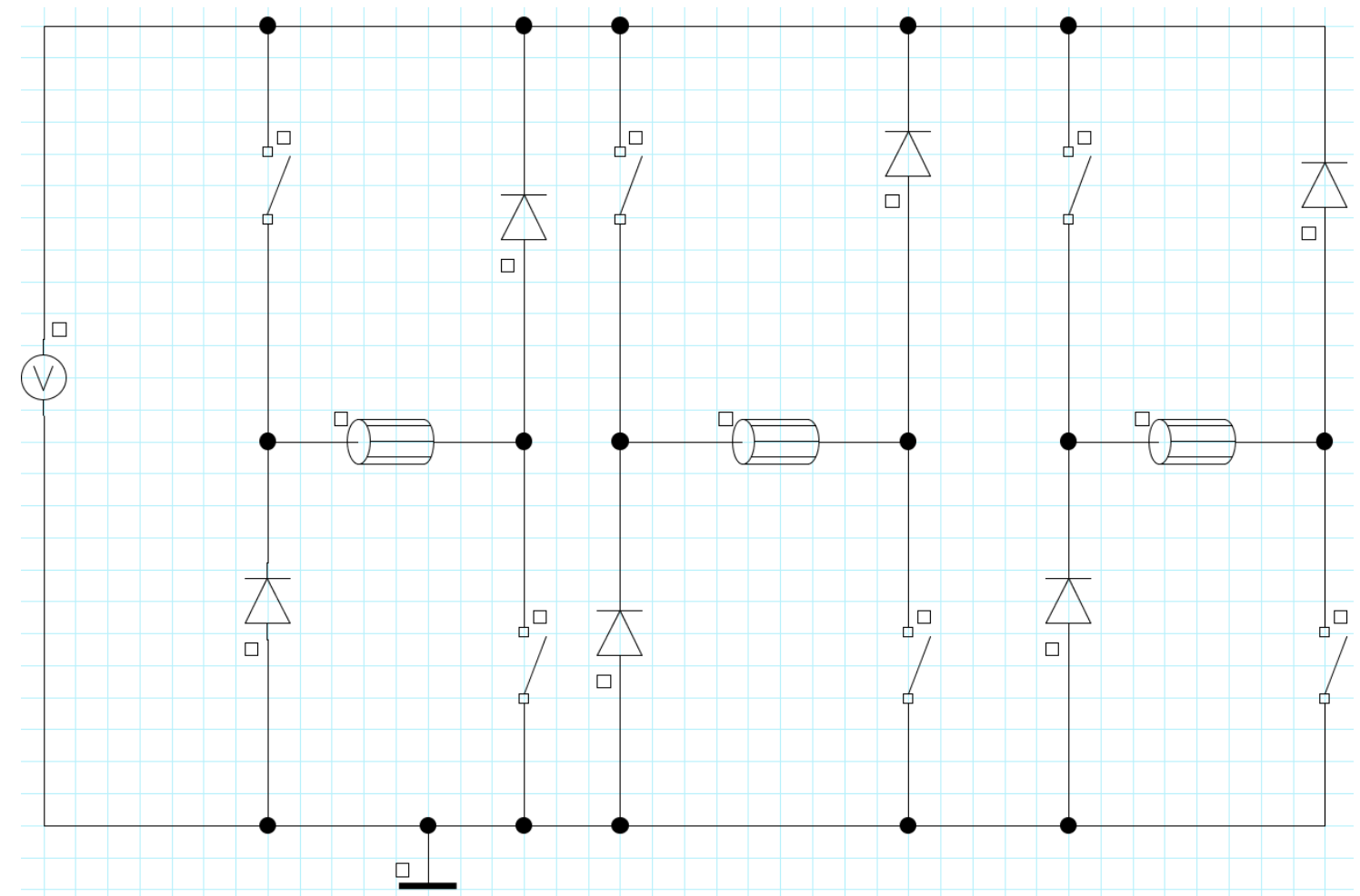

Figure 4.4: The simulation power converter.

In addition, a current sensor is attached to each phase to implement a hysteresis control for the phase current. The controller will regulate the phase currents to keep them within a 5\% margin of the reference current. The converter reference current was chosen to be $\boldsymbol{i}_{\boldsymbol{r} \text { ef }}=5 \mathrm{~A}$, with a current density limit of $(4.7-5.4) \mathrm{A} / \mathrm{mm}^{2}$ for a Totally Enclosed not Ventilated (TENV) SRM [9], while the converter DC voltage is kept at 
$V_{d c}=50 \mathrm{~V}$. The power converter for the new motor is shown in Fig. 4.5. It has the same models for the switches and the diodes as those of the power converter. The phase windings for the primary and the secondary are placed in two slots, as shown in Fig. 4.2, in accordance to their number of turns. Further, the resistance is also divided between the two windings.

For each simulation set, the operational parameters for the motor should be specified encompassing the mechanical speed, the turn-on and turn-off angles, and the reference current for the control. The electrical and the mechanical variables were recorded for each mechanical degree of the motor rotation.

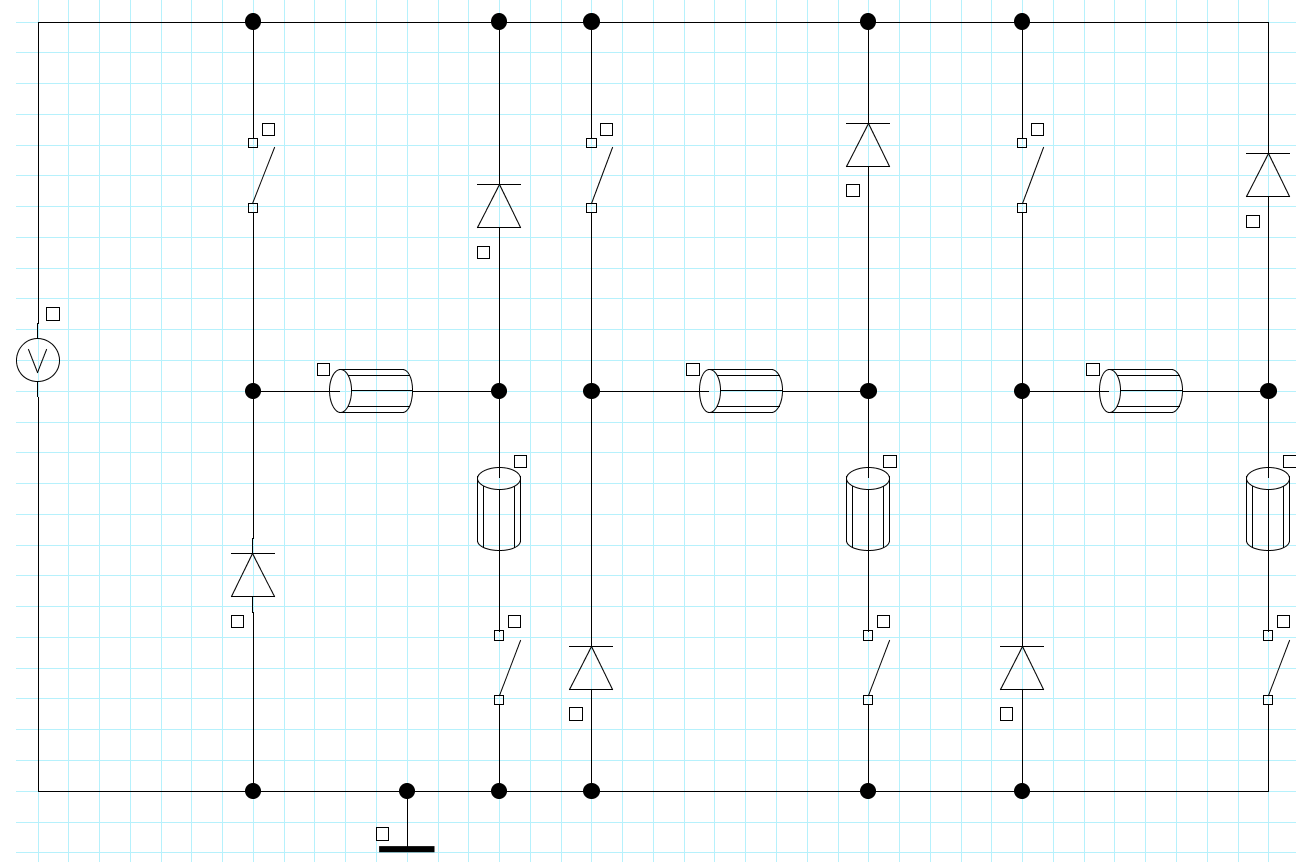

Figure 4.5: The new motor power converter.

\subsection{FEA Simulation Results}

A simulation analysis is performed to provide a comparison between the conventional motor models and the new motor models for different operational conditions. Improvement is expected in the average torque production and torque ripple 
minimization in the high speed regions. The proposed winding configuration is capable of improving the SRM torque-speed (T- $\omega$ ) characteristics by allowing a higher torque to be achieved at high speeds. The torque production for the typical SRM at high speeds is low due to the high back EMF voltage, and the need for early turn-off angles which shorten the time associated with each electrical cycle.

The demagnetization capability of the new winding topology is compared with that of the conventional SRM in Figs. 4.6-4.8, where the same turn-on and turn-off angles are applied for both models.

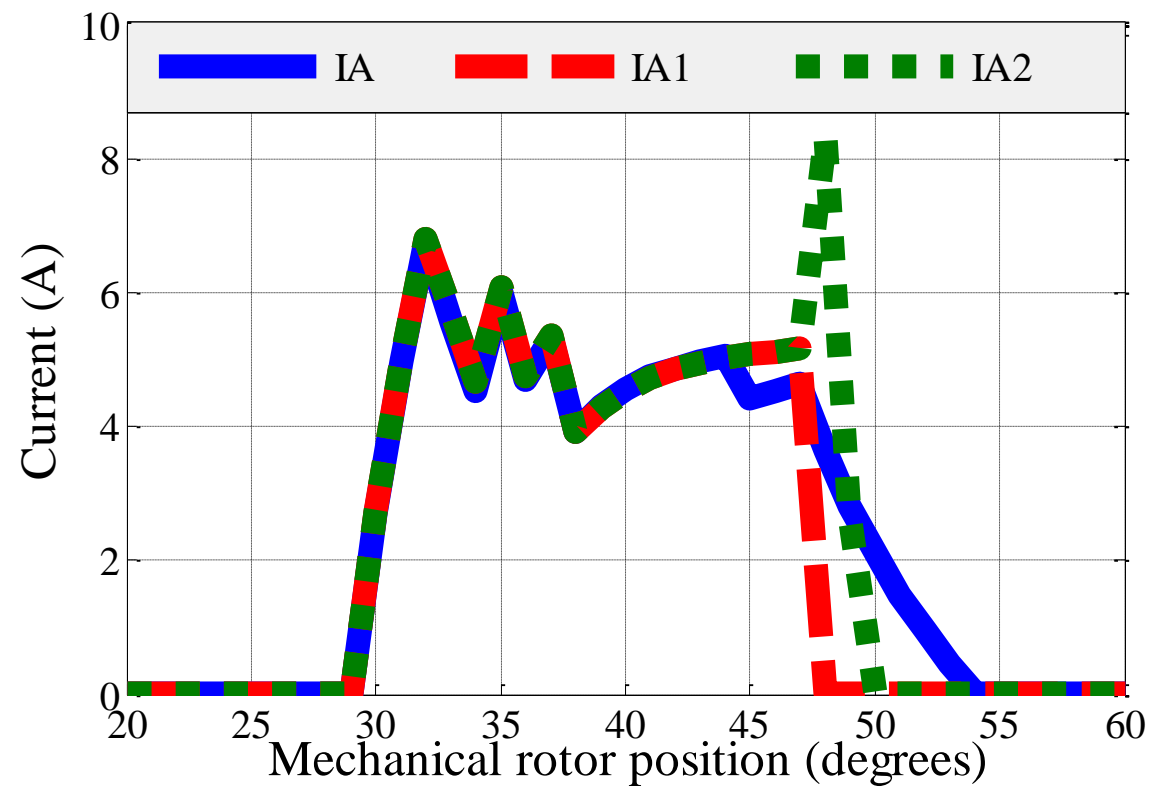

Figure 4.6: Phase A current comparison at low speed (500 rpm).

The currents in the conventional machine are compared with the currents in the primary and the secondary windings at different speeds, where IA, IA1, and IA2 are the conventional windings Phase A current, the primary Phase A current in the new topology, and the secondary Phase A current in the new topology respectively. 


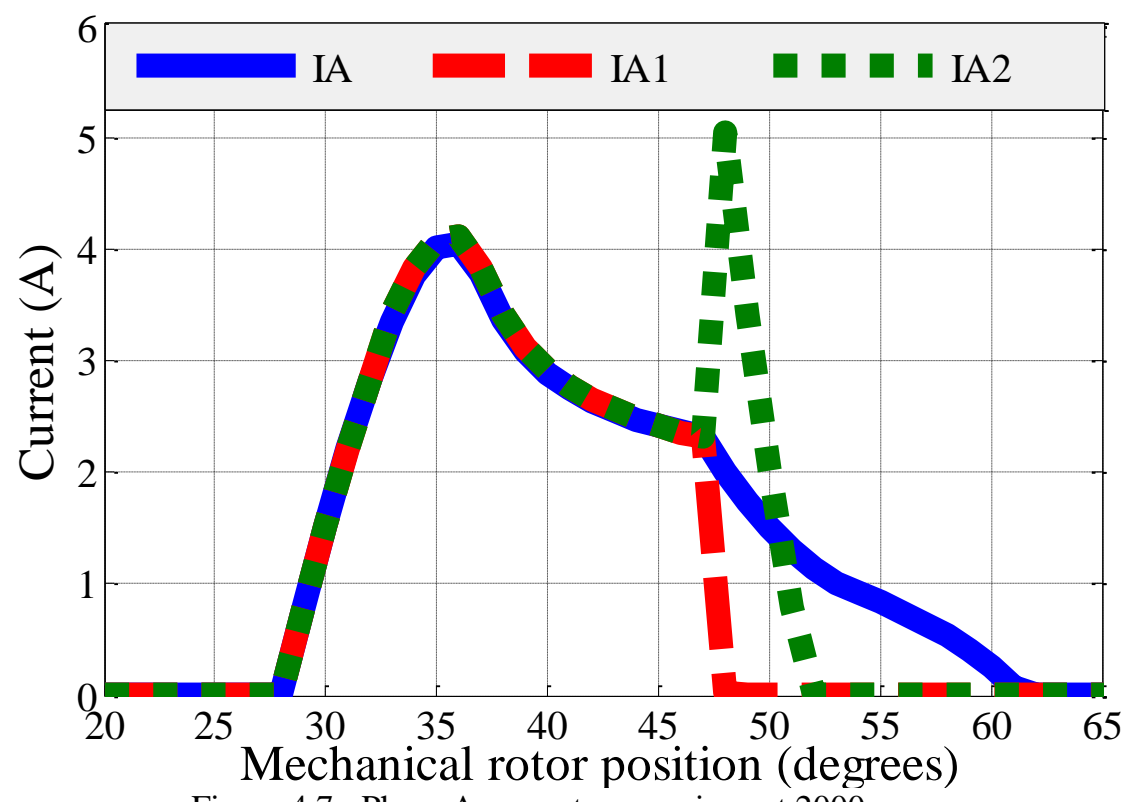

Figure 4.7: Phase A current comparison at $2000 \mathrm{rpm}$.

It is clear that the current in the secondary windings will jump instantaneously after turning the switch OFF and it will then be discharged quickly as shown in Figs. 4.64.8. This jump in the current is governed by the turns ratio between the secondary $\left(N_{2}\right)$ and the primary $\left(N_{1}\right)$ windings and it is an important consideration for the converter design to account for these instantaneous high values in the components ratings. At low speeds, the impact of the fast demagnetization is not significant since there is sufficient time for the phase current to be demagnetized. However, as the speed increases, the impact of the fast demagnetization is well demonstrated in Figs. 4.7 and 4.8. 


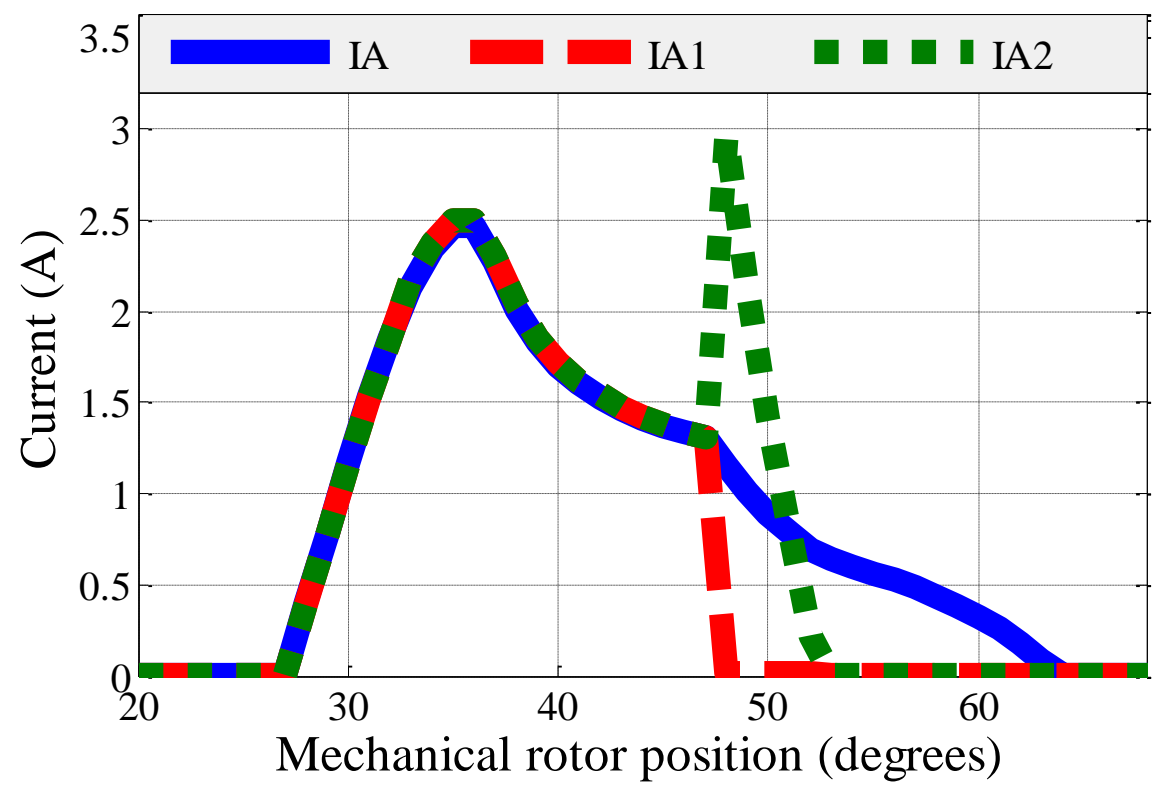

Figure 4.8: Phase A current comparison at $4000 \mathrm{rpm}$.

Clearly, the proposed configuration has the advantage of avoiding operation into the negative torque region since the current could be demagnetized faster. Thus the current can be allowed to continue to increase for a longer period of time in the proposed configuration. Since the demagnetization time is shortened, the proposed configuration makes it possible to produce a higher torque and possibly end up with enhanced fieldweakening capability [6] [32].

In order to investigate the effect of the new winding configurations on the phase voltages the voltage across the Phase A winding, during the magnetization and demagnetization, is compared with those of the primary and the secondary winding configuration in Fig. 4.9, where the turns ratio is responsible for defining the voltage levels across the primary and the secondary windings.

It is essential to study the effect of extinguishing the current instantaneously in the primary windings and directing the flux to the secondary windings on the power converter and the SRM ratings. The sudden change in the phase current imposes a high 
voltage across the primary windings, along with fast transients on the diode current as shown in Figs. 4.9 and 4.10.

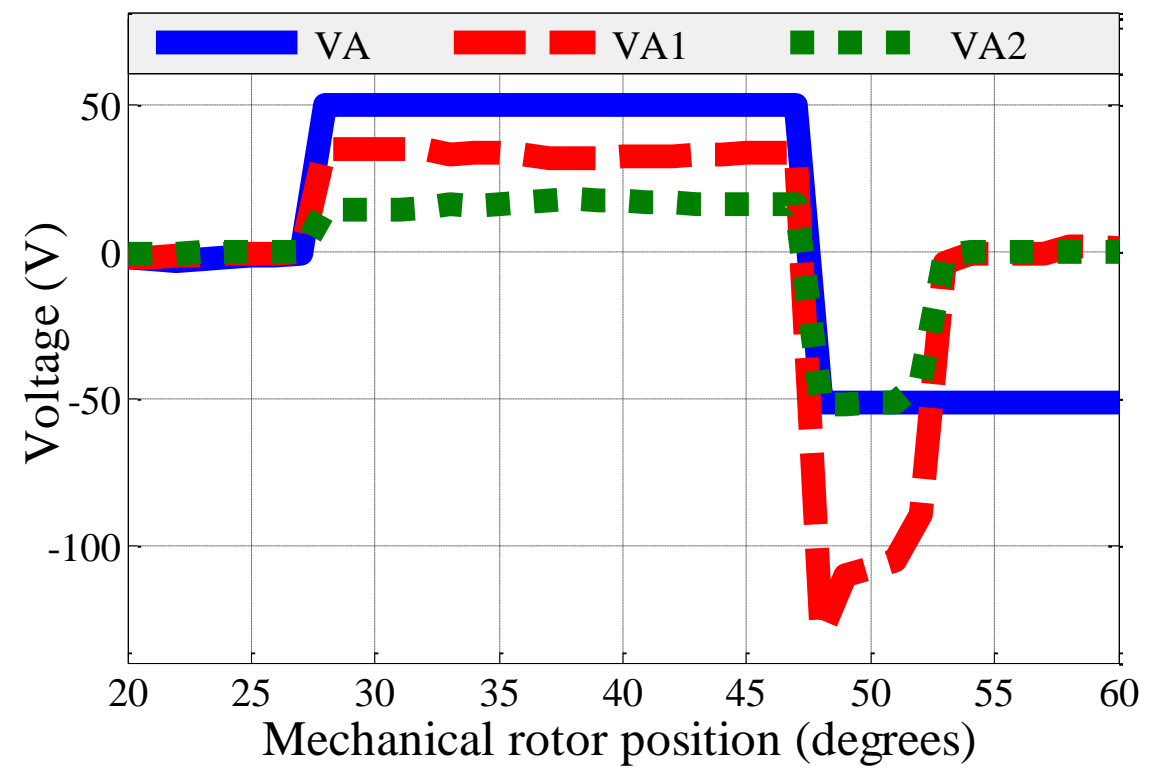

Figure 4.9: Phase A voltages comparison at $2000 \mathrm{rpm}$.

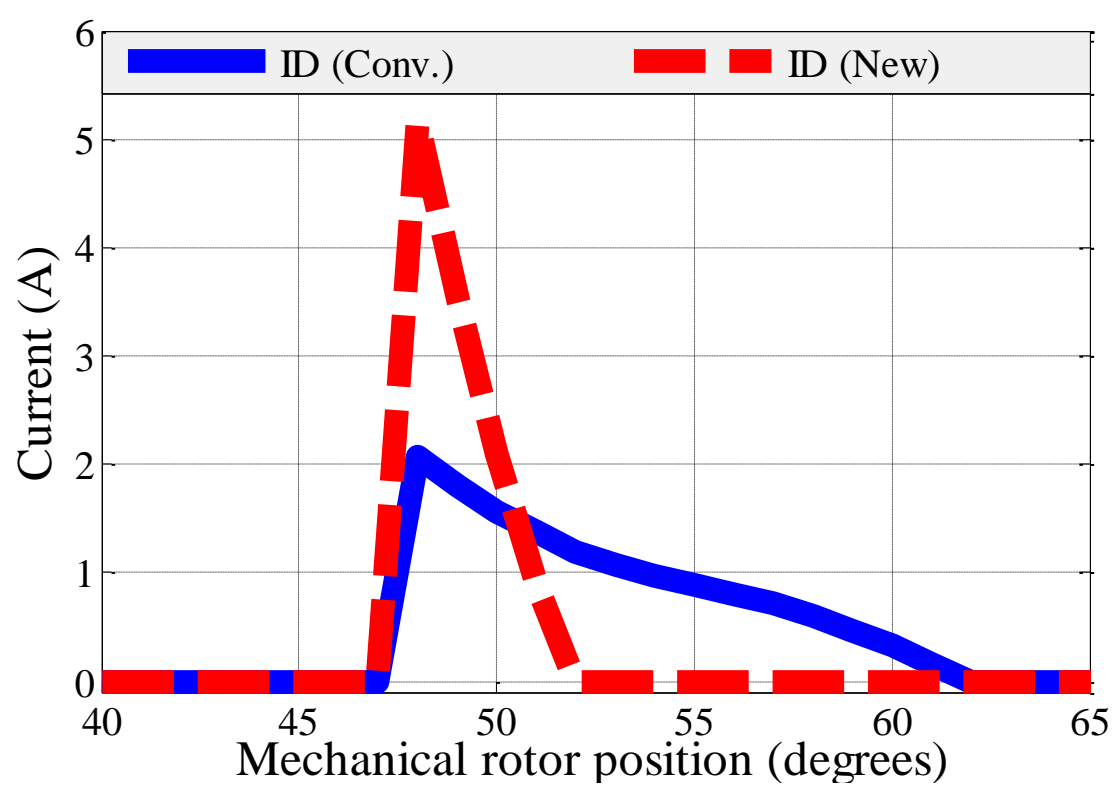

Figure 4.10: Diodes currents comparison at $2000 \mathrm{rpm}$.

Fig. 4.11 compares the voltages across the diodes for two configurations. It is clear from Fig. 4.11 that the duration for the demagnetization in the proposed configuration is shorter than that in the conventional machine. 


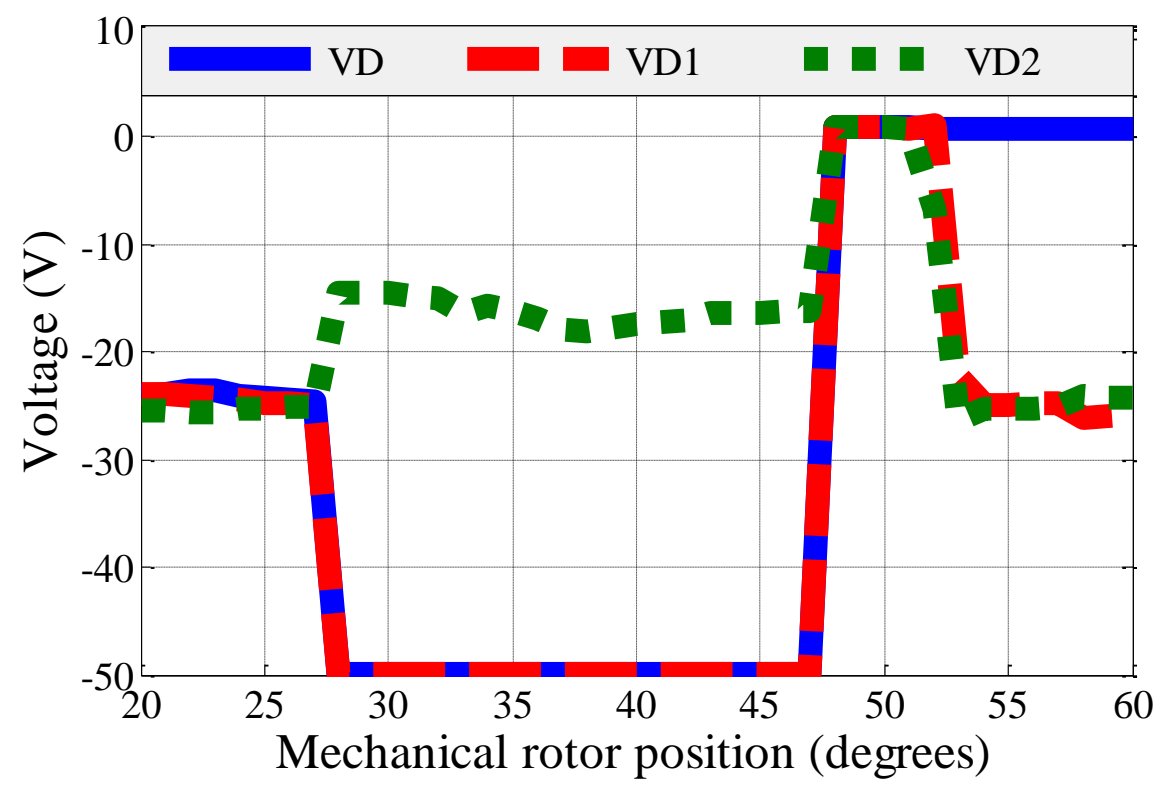

Figure 4.11: Diodes voltages at $2000 \mathrm{rpm}$.

\subsection{Simulation Results}

The simulation results demonstrate the improvement in the average torque production and the torque ripple minimization. The simulation was run for different mechanical speeds and the average torque was obtained for each speed. An optimization process was carried out to determine the optimum turn-on and turn-off angles for each speed that corresponds to the maximum possible average torque within the current density constraints. The current density criteria was considered by limiting the RMS current for each phase to the maximum value that the motor can tolerate regarding the TENV cooling used with the actual motor, which can be estimated as follows:

$$
J=\frac{I_{r m s} N}{2 K_{S} A}
$$

where, $\mathrm{J}$ is The current density $\mathrm{A} / \mathrm{mm}^{2}$ that should not exceed $5 \mathrm{~A} / \mathrm{mm}^{2}, I_{r m s}$ is the RMS current for each phase, $\mathrm{N}$ is the number of turns for each phase (150 turns for the case study machine), $K_{s}$ is the slot-fill factor that represents the portion of slot filled with 
the windings, $K_{s}=0.453$ for the motor under study, and $\mathrm{A}$ is the slot area in $\mathrm{mm}^{2}, \mathrm{~A} \approx$ $105 \mathrm{~mm}^{2}$.

The maximum RMS current corresponding to the these values was found to be $I_{r m s} \approx$ 2.6 $\mathrm{A}$, given that each phase has two parallel paths of conductors which was represented by the factor 2 in the denominator in Eqn. 4.1 .

The torque-speed curves for the case study before and after implementing the new winding topology are shown in Fig. 4.12, while the power comparison is presented in Fig. 4.13. The optimum values for a turn-on and turn-off angles comparison is shown in Table 4.2. It is expected that the turn-off angle will be greater with the new configuration, but it was also found that turn-on will also be slightly greater.

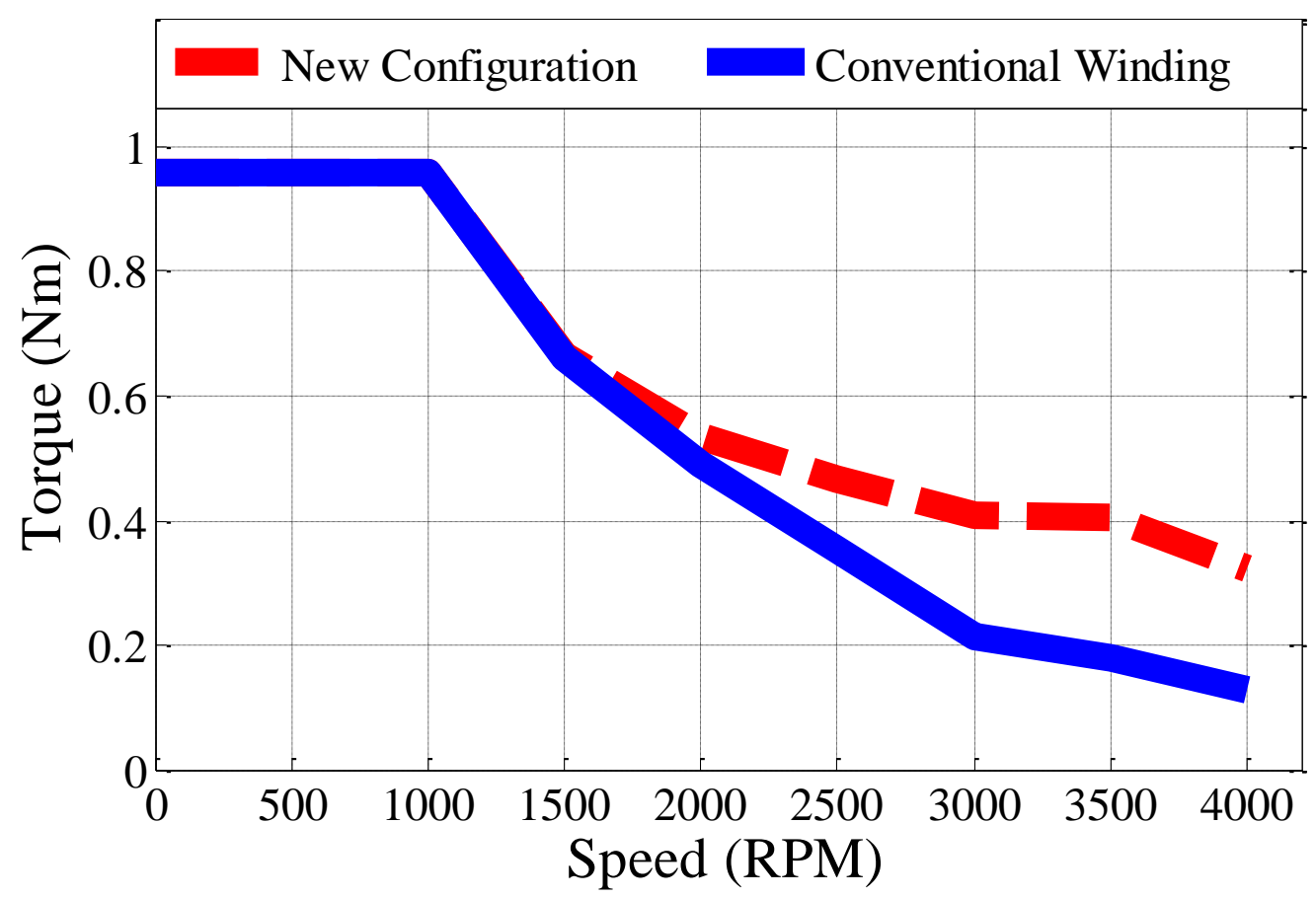

Figure 4.12: Torque- speed curves comparison. 


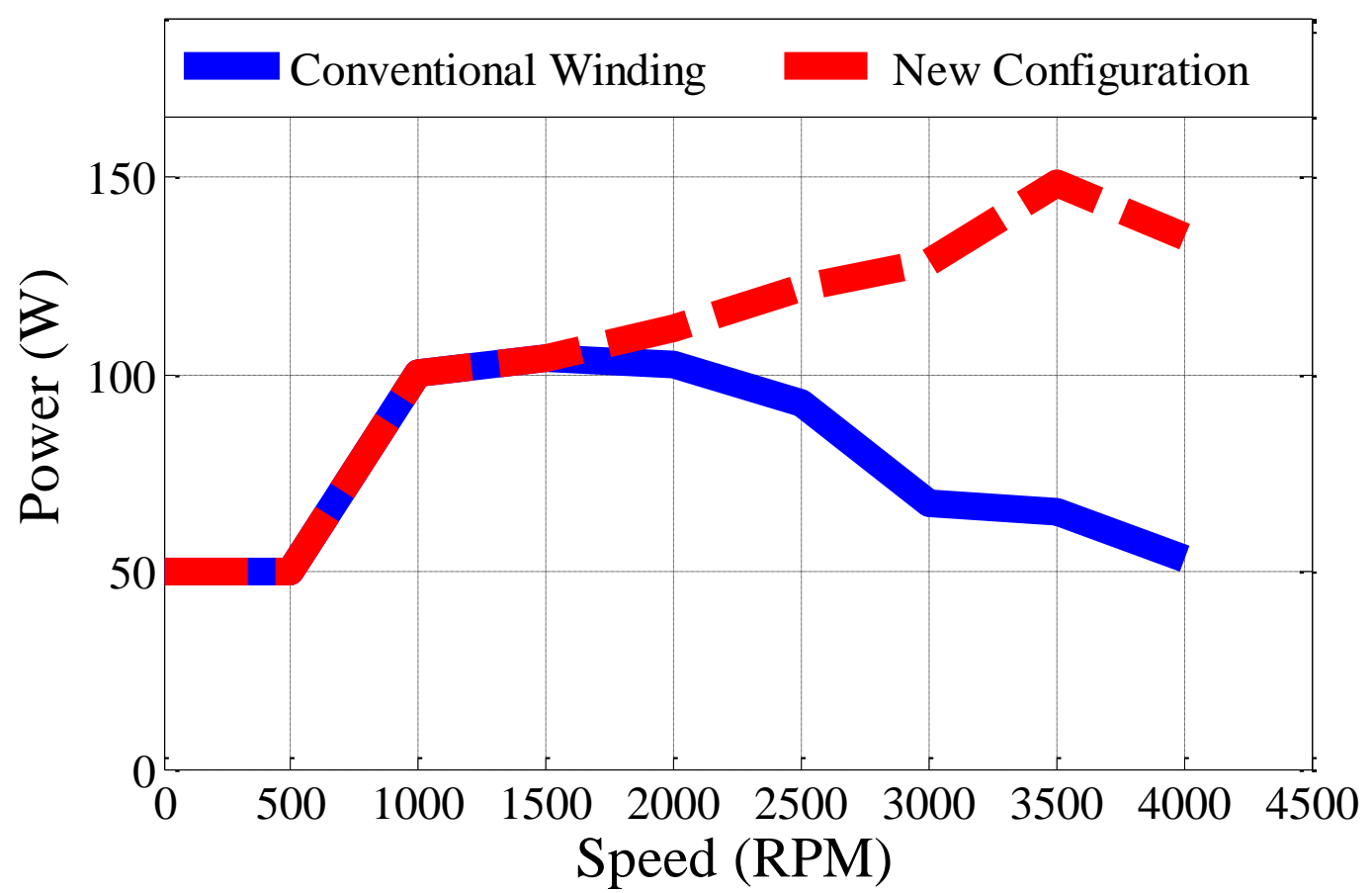

Figure 4.13: Power - speed curves comparison.

Table 4.2: Turn-on and turn-off angle comparison.

\begin{tabular}{ccccc}
\hline \multirow{2}{*}{ Speed } & \multicolumn{2}{c}{ Conventional } & \multicolumn{2}{c}{ New Motor } \\
& Turn-on & Turn-off & Turn-on & Turn-off \\
\hline 500 & 0 & 140 & 0 & 140 \\
1000 & 0 & 140 & 0 & 140 \\
1500 & -20 & 130 & -20 & 130 \\
2000 & -64 & 128 & -70 & 170 \\
2500 & -64 & 124 & -70 & 170 \\
3000 & -66 & 120 & -70 & 170 \\
3500 & -66 & 120 & -70 & 170 \\
\hline 4000 & -66 & 112 & -70 & \\
\hline
\end{tabular}


The proposed topology can result in torque ripple minimization as the current will be sustained for longer time and better current controllability can be achieved for a higher DC bus voltage applied to the case study motor, as shown in Figs. 4.14 and 4.15, respectively.

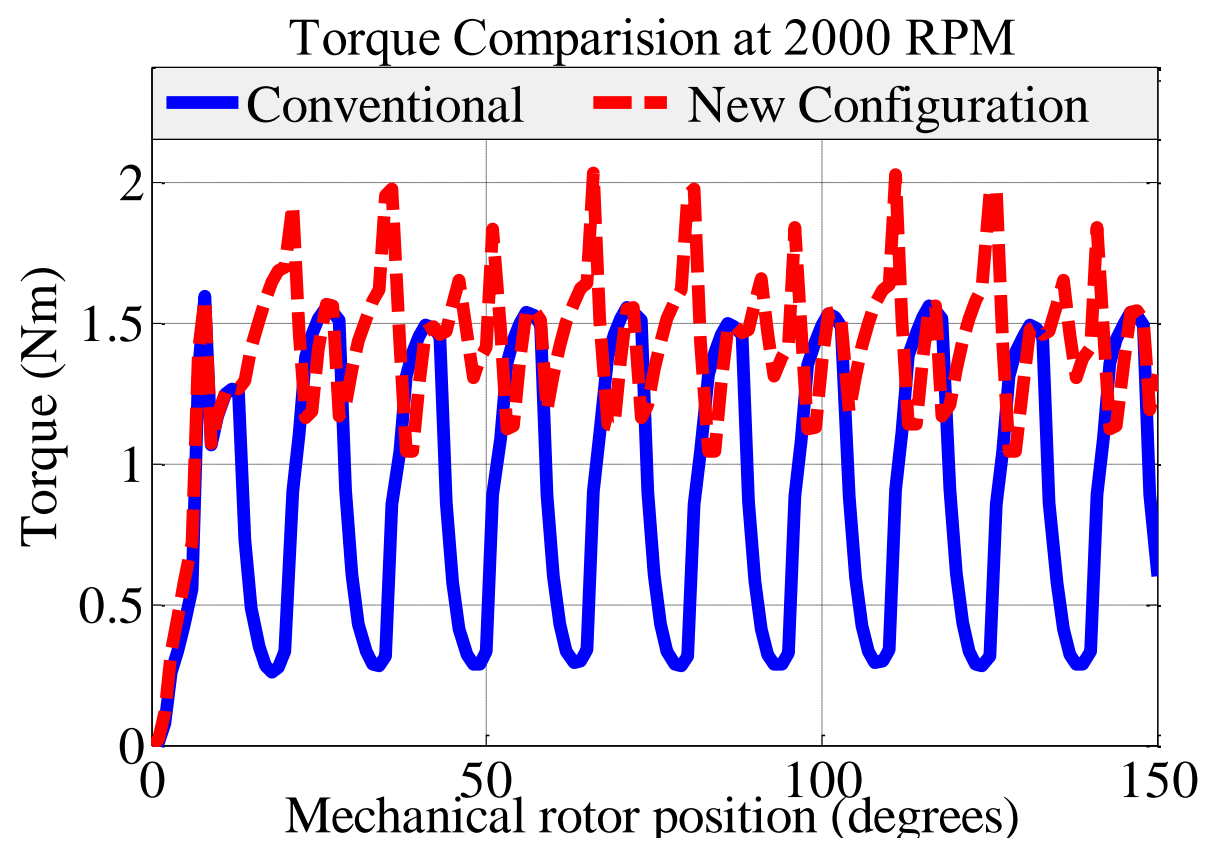

Figure 4.14: Torque comparison at $2000 \mathrm{rpm}$.

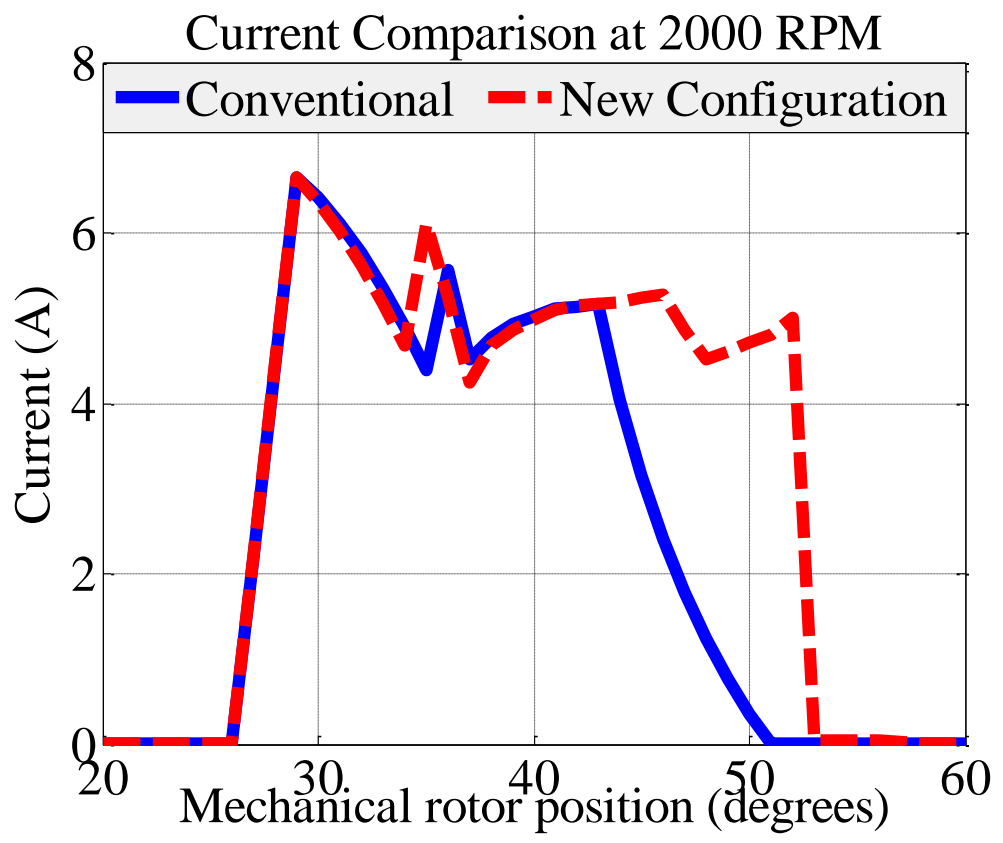

Figure 4.15: Phase A current Comparison at 2000 rpm. 


\subsection{Leakage Inductance Simulation}

The autotransformer behavior for the windings is based on the ideal coupling assumption, which is not practically the case. For the sake of comparison, and to investigate the effects of non-ideal coupling between the two windings, the voltage across the primary windings during demagnetization is compared for the cases of ideal and nonideal coupling.

Fig. 4.16 presents the primary voltage for the ideal coupling with a magnetizing inductance of $10 \mathrm{mH}$, a DC bus voltage of $50 \mathrm{~V}$, and a turns ratio of 2:1 for the primary to the secondary.

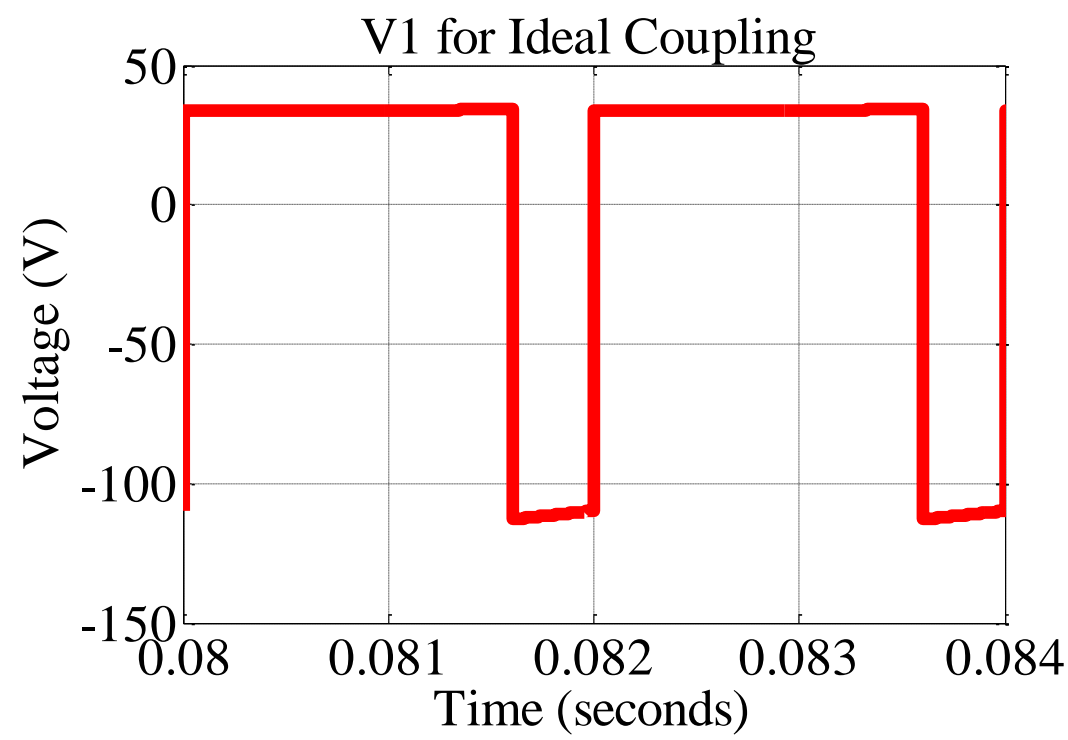

Figure 4.16: Primary windings voltage for ideal coupling.

The same autotransformer with a leakage inductance of $1 \%$ of the magnetizing inductance is simulated, and the voltage across the primary windings is presented in Fig. 4.17. The effect of non-ideal coupling results in voltage spikes in the primary windings and the electronic switch $\left(S_{2}\right)$ connected to that winding terminals, as shown in Fig. 4.18. The voltage spike should be considered in the components ratings for the driver. The leakage inductance theoretically results in an instantaneous change in the flux linked to 
the primary winding, but for the real application even with leakage inductance the voltage peak will be affected by the switching time and the switch impedance; that is, an infinite peak voltage corresponds to ideal switching with infinite impedance for the switch in the off state.

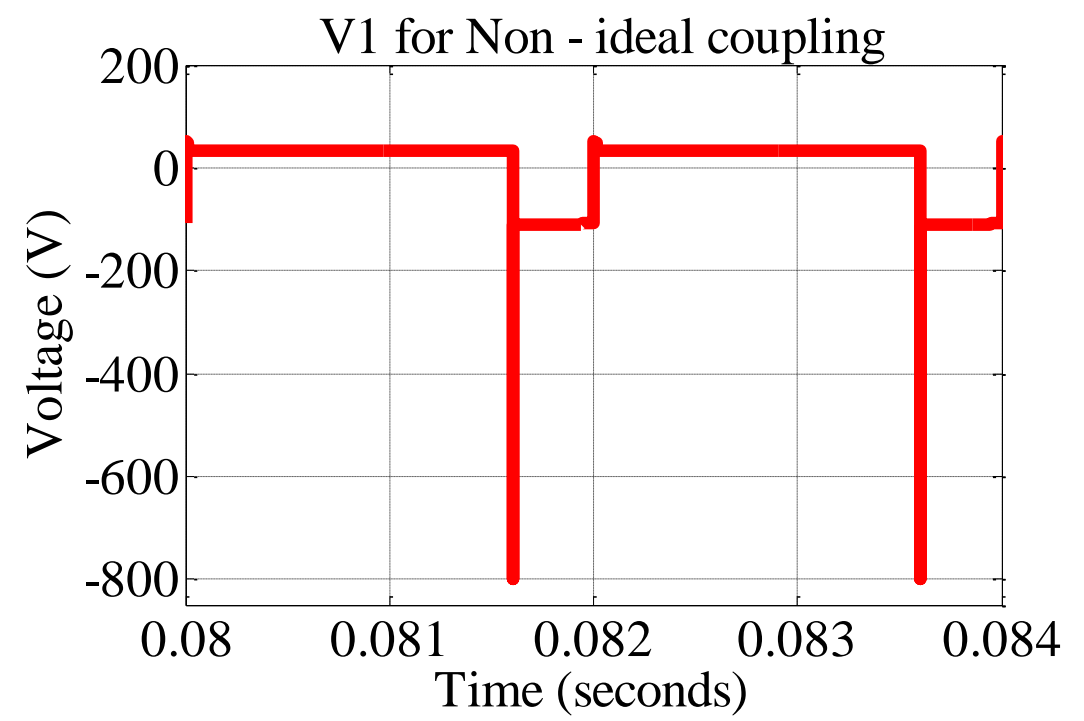

Figure 4.17: Primary windings voltage for non-ideal coupling.

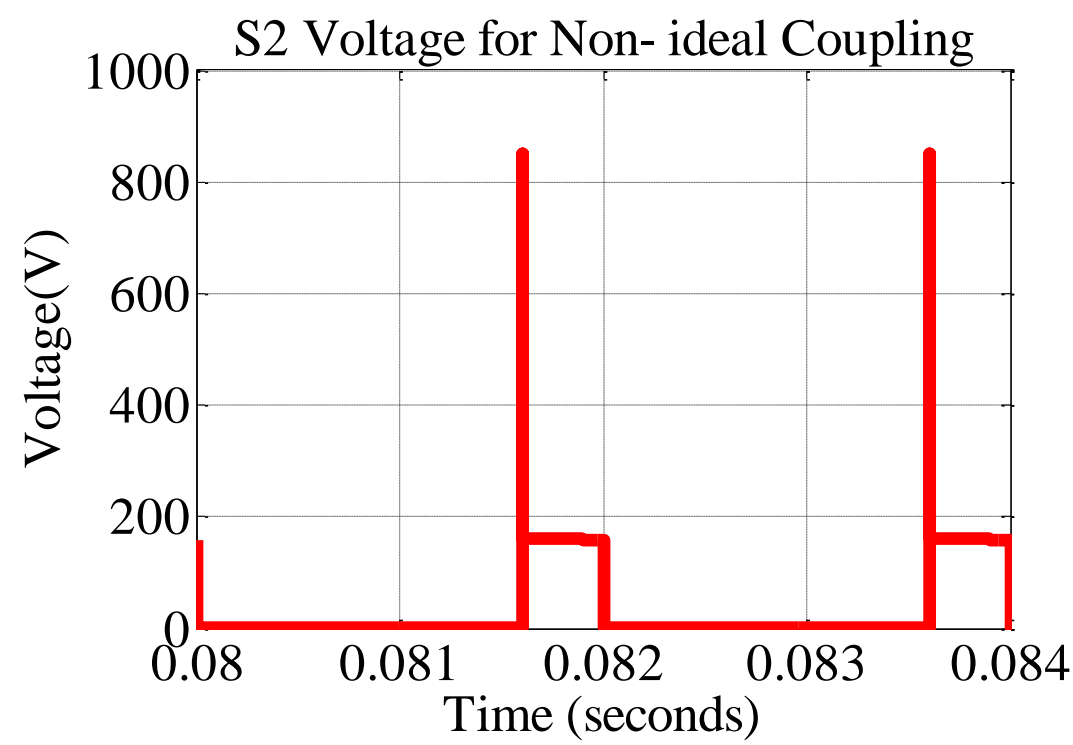

Figure 4.18: S2 Voltage for non-ideal coupling.

The voltage across the other switch (S1) will be the same for both the ideal and nonideal coupling cases as shown in Fig. 4.19. 


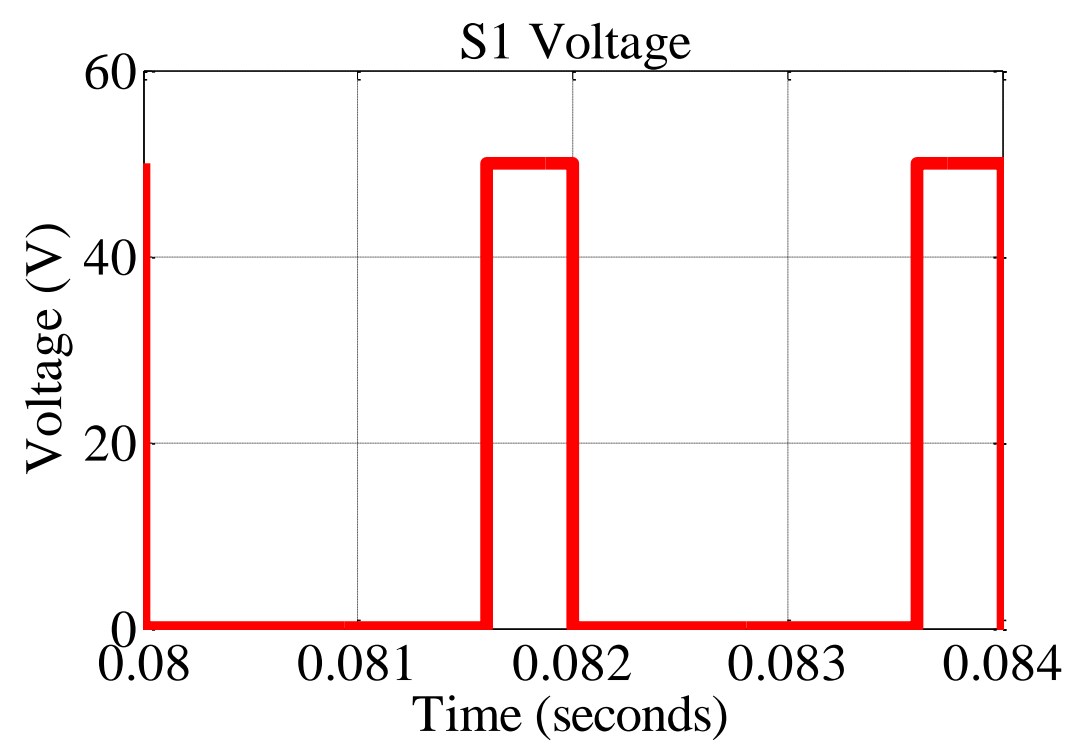

Figure 4.19: S1 Voltage for ideal and non-ideal coupling.

\subsection{Conclusion}

This chapter investigated the dynamics of implementing the auto-transformer winding topology on the simulation level. SRM models were built in reference to a commercially available motor to realize a fair comparison between the conventional and the proposed new motor. The simulation results showed promising advantages of implementing the proposed work of the thesis. The dynamics of the power converter operation resulting from the leakage inductance of the new windings were studied with a separate simulations set. The hardware setup should take the leakage inductance into consideration for proper operation of the new motor. 


\section{CHAPTER V}

\section{HARDWARE IMPLEMENTATION AND EXPERIMENTAL RESULTS}

\subsection{Introduction}

The simulation results from Chapter 4 verified the advantage of the new winding topology in improving the average torque as well as the torque ripple profile for SRMs at high speed regions. The control and dynamics of the power converter were investigated after interfacing the driver model with the SRM FE model. The hardware implementation of the system is crucial to validate the simulation results. This chapter discusses the hardware developments and the experimental results in details.

\subsection{The Conventional Motor Setup}

A conventional SRM and its associated asymmetric converter, as shown in Fig.

5.1, were used as a reference to be compared with the SRM having the new winding topology. The competency of the FEA model of Chapter 4 was verified experimentally on a $100 \mathrm{~W}, 12 / 8$ SRM machine which was developed for a washing machine application. The percentage error between the FEA model and the experimental setup was found to be within $10 \%$ for different loading conditions, as depicted in Table 5.1. The setup is composed of the SRM, the power converter, the dSpace microAutoBox [33] controller, and the Magtrol dynamometer [34], which was coupled to the SRM shaft to apply different loading conditions to the motor. 


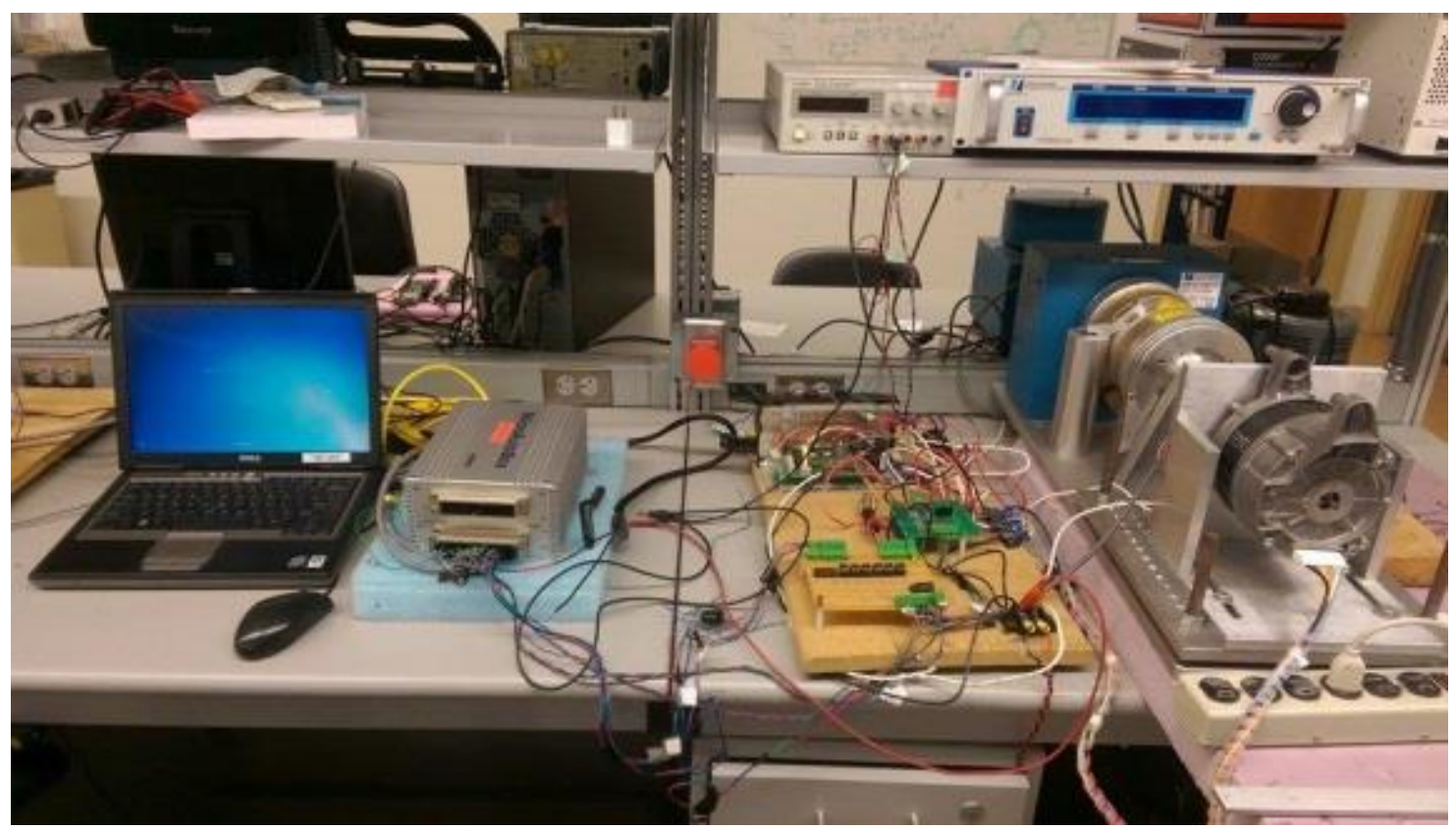

Figure 5.1: Conventional SRM experimental setup.

Table 5.1: Finite element model and actual setup torque comparison for different operating conditions.

\begin{tabular}{cccc}
\hline Speed $(\mathbf{R P M})$ & FEM Torque $(\mathbf{N m})$ & Actual Machine Torque (Nm) & Error (\%) \\
\hline \hline 500 & 0.96 & 0.92 & +4.35 \\
1000 & 0.96 & 0.92 & +4.35 \\
1500 & 0.662 & 0.701 & -5.56 \\
2000 & 0.491 & 0.53 & -7.36 \\
2500 & 0.354 & 0.32 & +10.63 \\
3000 & 0.214 & 0.22 & -2.73 \\
\hline
\end{tabular}

The power converter includes IGBTs (Insulated-gate bipolar transistors) as the electronic switches, diodes, and current sensors to provide the phase currents measurements to the controller. The mechanical position of the motor is tracked using an encoder attached to the motor shaft. These components will be discussed in the driver design section. 
The results obtained from the conventional setup regarding torque production are depicted in Table 5.1. The current waveforms for the conventional SRM are shown in Fig. 5.2 for a loading of $1.5 \mathrm{Nm}$ at a DC bus voltage of $90 \mathrm{~V}$. The current reference is dictated by a closed loop speed control to run the motor at $500 \mathrm{rpm}$. The speed is estimated from the encoder feedback that is also used to develop the switching commands according to the rotor position. Further, a hysteresis current controller is implemented to produce the desired phase currents.

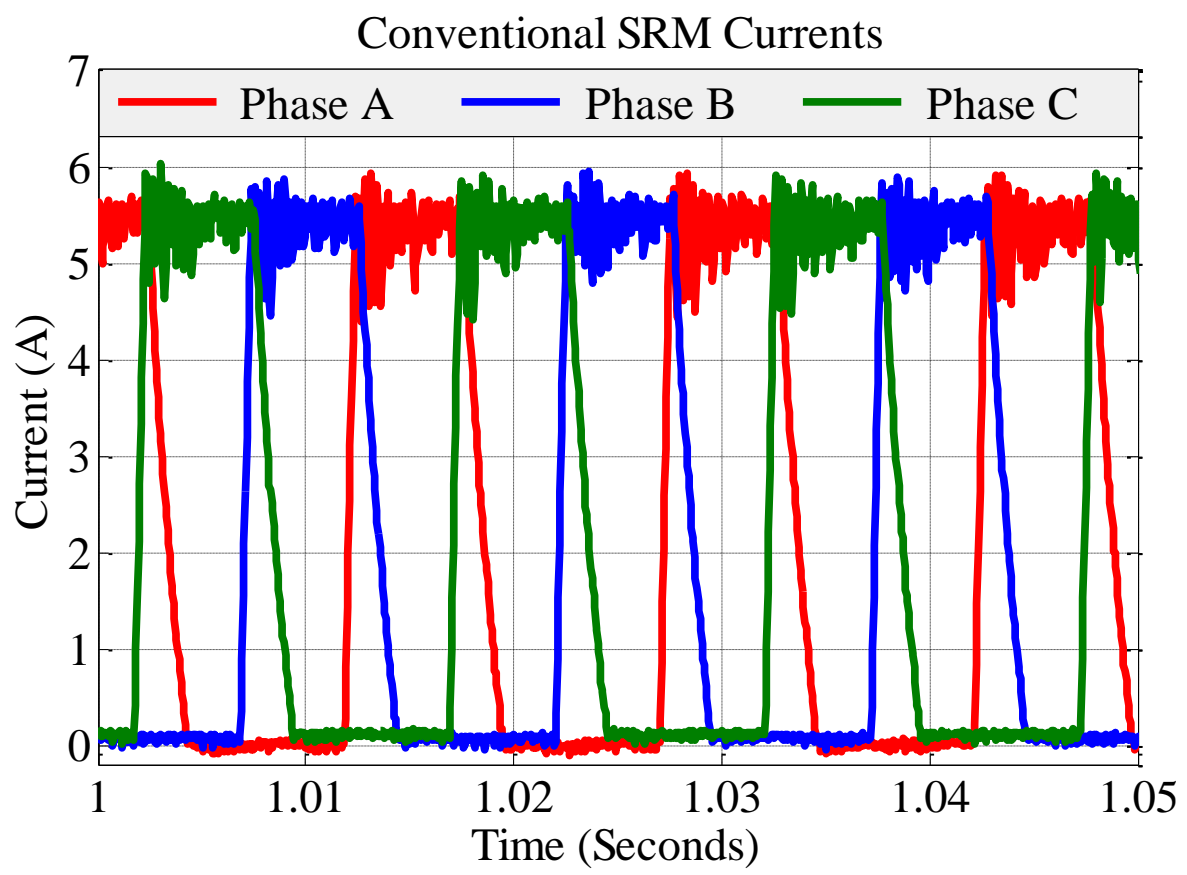

Figure 5.2: Phase currents of a conventional SRM operating at $500 \mathrm{rpm}$.

\subsection{The New Motor Development}

To establish a fair comparison the new winding topology was implemented on an SRM that has the same number of turns as those of the conventional one. A rewinding process was carried out to reconfigure the machine windings for the desired topology. For the case study motor, each phase has two sets of coils connected in parallel, and each coil is wound around two different stator poles connected in series. The pole winding is 
referred to as a conductor in the analysis that follows. Fig. 5.3 depicts a simplified diagram for the phase connection in the motor with the new winding configuration.

The main difficulty in reconfiguring the motor windings is connecting the primary windings in series and the secondary windings in series, taking into consideration that the two primary as well as the two secondary windings are located in two different stator poles.

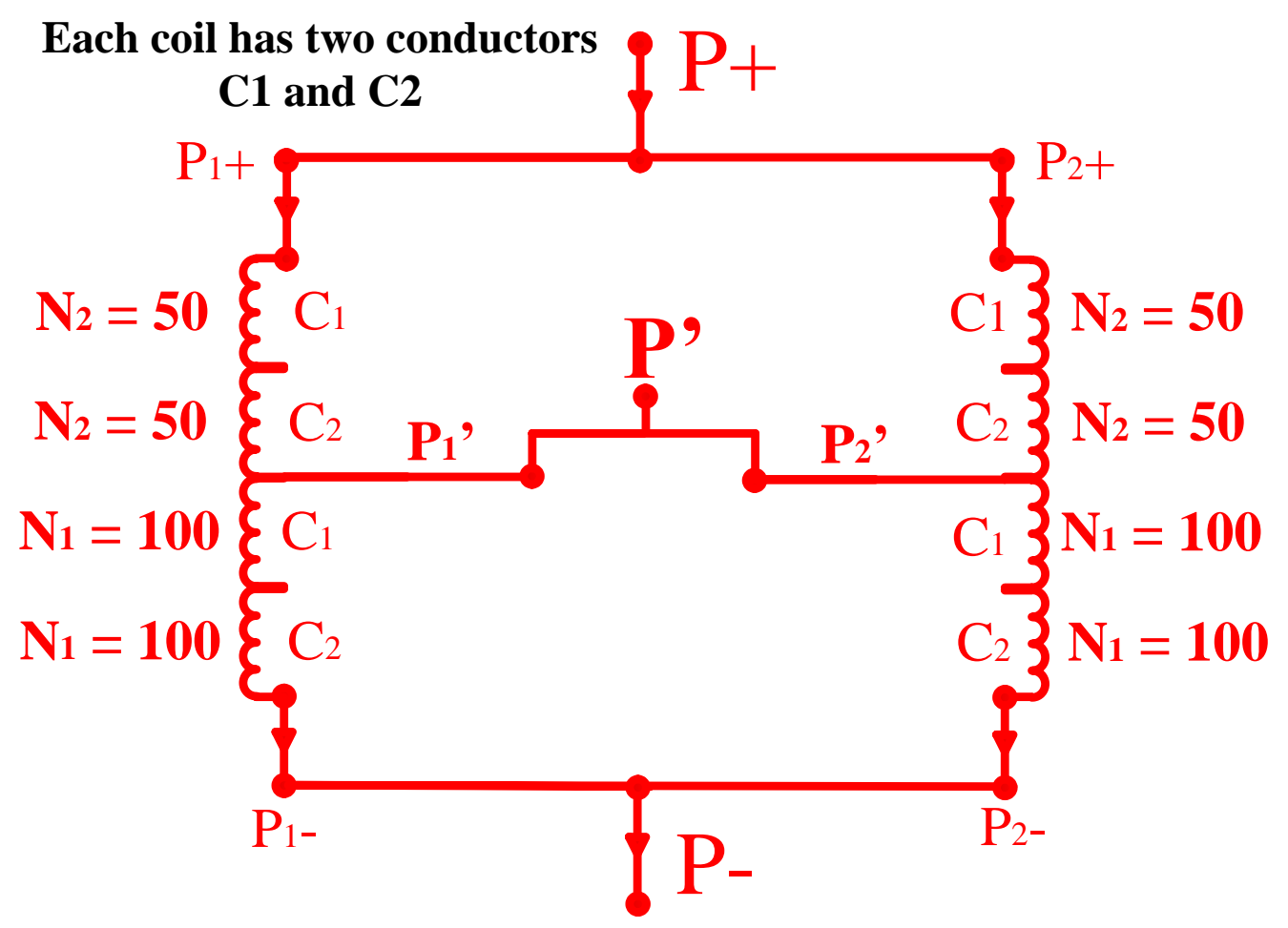

Figure 5.3: Winding connection of the new SRM.

Fig 5.4 illustrates the implementation of the new windings on the stator poles taking phase $\mathrm{B}$ as an example. The primary windings were placed at the top of the stator poles while the secondary windings were placed at the bottom. For each conductor (e.g. $B_{1}$ ) the two secondary windings are first connected in series and then the two primary windings are connected in series. Fig. 5.5 shows the SRM after the rewinding process, while the three leads of the phases are shown in Fig 5.6. 


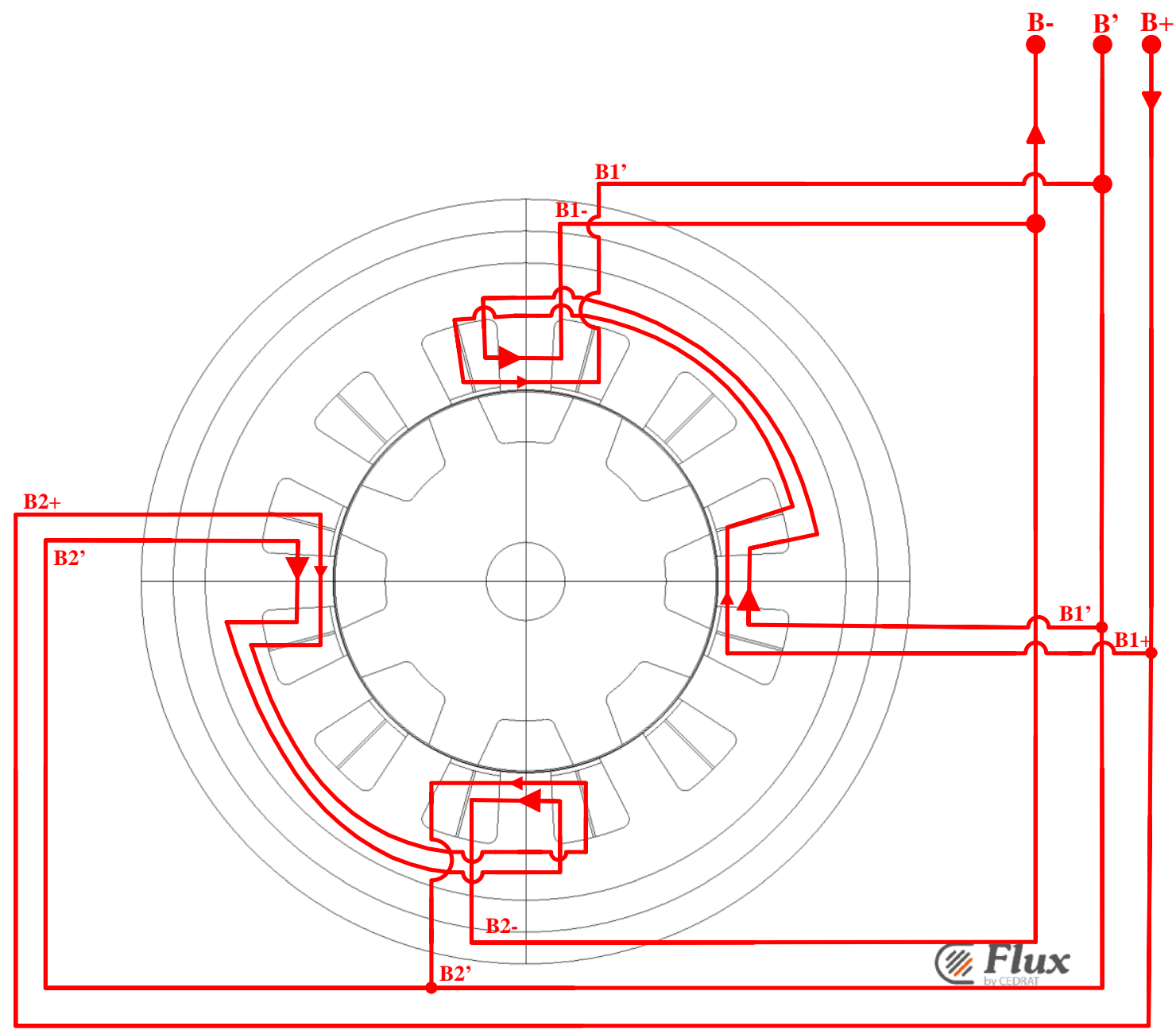

Figure 5.4: The new windings configuration of phase B.

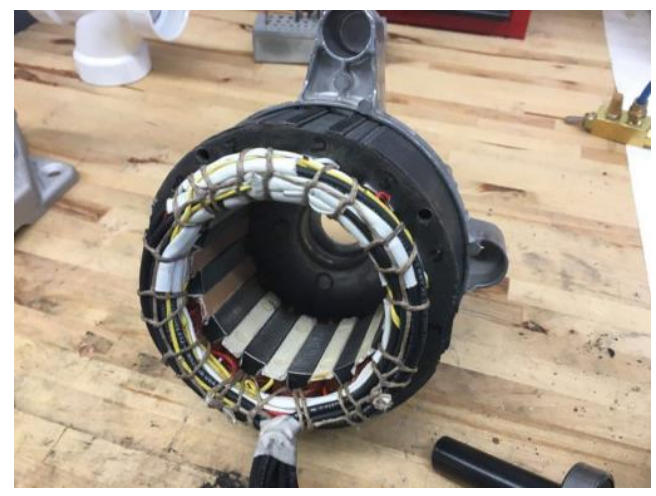

Figure 5.5: SRM motor after the rewinding.

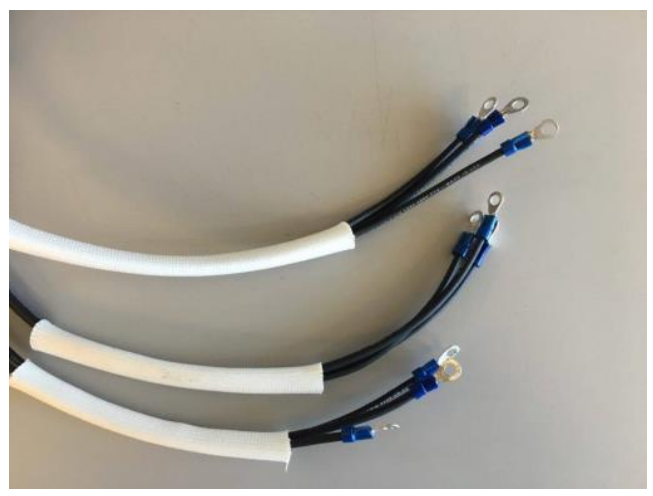

Figure 5.6: The three leads of each phase. 


\subsection{Power Converter Design}

\subsubsection{The Design Requirements}

The power converter for the new motor is designed, developed, and tested for the following requirements:

- Number of phases: 3 .

- DC bus voltage: $50 \mathrm{~V}$.

- Total RMS current provided by the DC Bus: 5 A.

- Power ratings: $200 \mathrm{~W}$.

- $\quad$ RMS current per phase: $2.6 \mathrm{~A}$.

- Switching frequency: $30 \mathrm{kHz}$.

- Rated (corner) speed: $1000 \mathrm{rpm}$.

- Maximum speed: $4000 \mathrm{rpm}$.

The power converter consists of a power circuit and a control circuit, discussed next.

- The Power Circuit

For the power circuit, a DC bus voltage of $50 \mathrm{~V}$ will be provided to the driver with a nominal RMS current of 5 A. The power circuit components should be able to handle the power ratings of the driver; that is:

- A maximum demagnetization phase voltage of $150 \mathrm{~V}$.

- A maximum peak current of 10 A during the demagnetization time.

- A maximum RMS current of 5 A.

- A maximum operation at a switching frequency of $30 \mathrm{kHz}$. 
The power circuit includes the IGBTs and diodes required to meet the design requirements.

- The Control Circuit

A $5 \mathrm{~V}$ DC supply will be used to power the control circuit. The control circuit consists of:

- The Gate drivers: The gate driver is responsible for driving the IGBTs according to the switching signals provided by the dSpace microAutoBox controller. The gate driver circuitry has an optocoupler chip to isolate the received control signal from the IGBT side, for the sake of protection. Moreover, the circuitry boosts the voltage level of the switching signal to meet with the IGBT driving signal requirements.

- Buffer: The control circuit of the power converter buffers three different signals; namely, the switching signals developed by the dSpace controller, the position measurements provided by the encoder, and the current measurements signals from the phase current sensors.

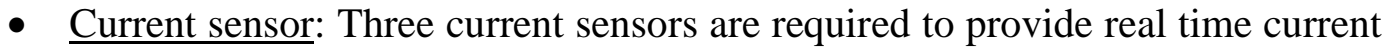
measurements to achieve current control.

The block diagram provided in Fig. 5.7 characterizes the power converter design that includes the power and the control circuit, along with the dSpace controller signals associated with the phase windings. The current sensors are connected in series with the secondary windings to measure the phase current during magnetization and demagnetization. 


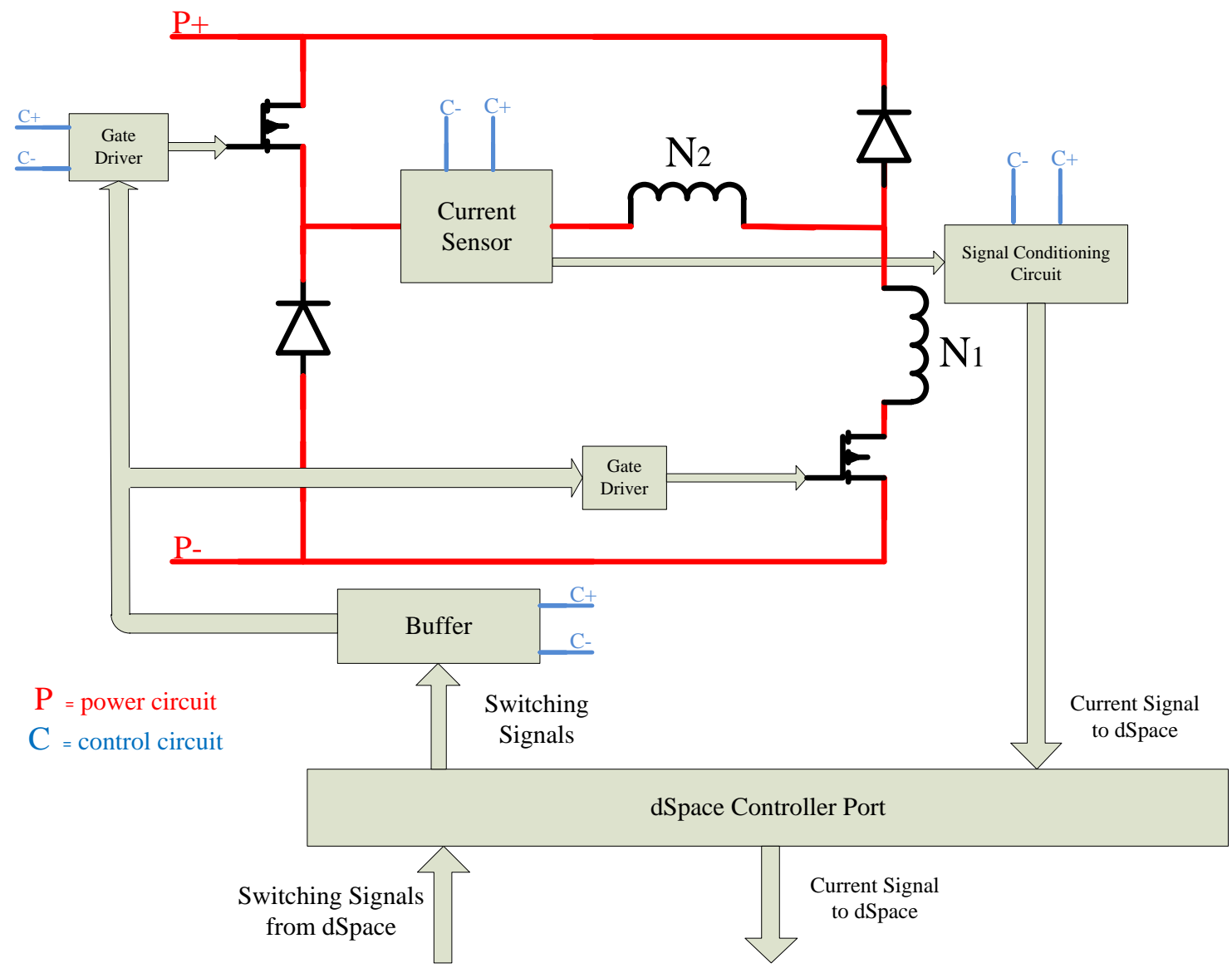

Figure 5.7: The block diagram of the SRM driver circuit.

\subsubsection{Printed Circuit Board (PCB) Design for the SRM Driver}

The second stage in developing the power converter following the defined requirements involves the creation of the schematics and the printed circuit board (PCB) designs for the driver circuitries. The main objectives of the circuit design are to meet the driver requirements, with an optimized layout and component use. A comprehensive schematic that includes the power and control circuitries is shown in Fig. 5.8. The schematic illustrates the main power circuit, buffer circuits, gate driver circuits, a current sensor, signal conditioning circuits for the current signals, and connectors to interface with the dSpace controller. Detailed schematics for these parts of the driver are presented next. 


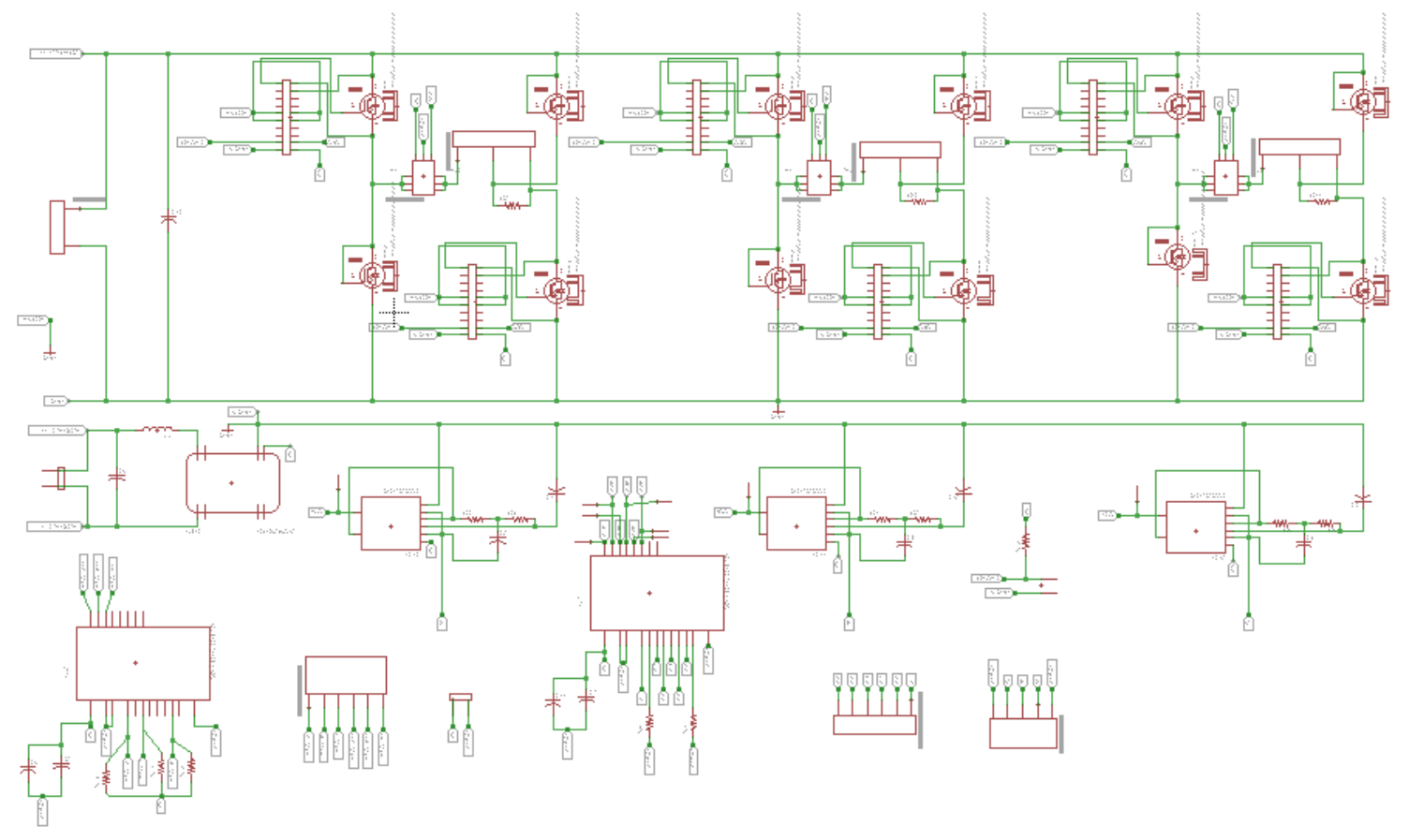

Figure 5.8: Power converter schematic design.

- Buffer Circuit for PWM Signal: The switching PWM signals are buffered to enhance the signal quality and to isolate the dSpace controller from the gate driver circuitry. The schematic for the buffer circuit is shown in Fig. 5.9. The signals $S_{1}$ to $S_{6}$ are provided by dSpace. The buffer conditions these signals and outputs $S_{1} B$ to $S_{6} B$ channels to the gate drivers. Similar buffering circuitry was used for the encoder signals. 


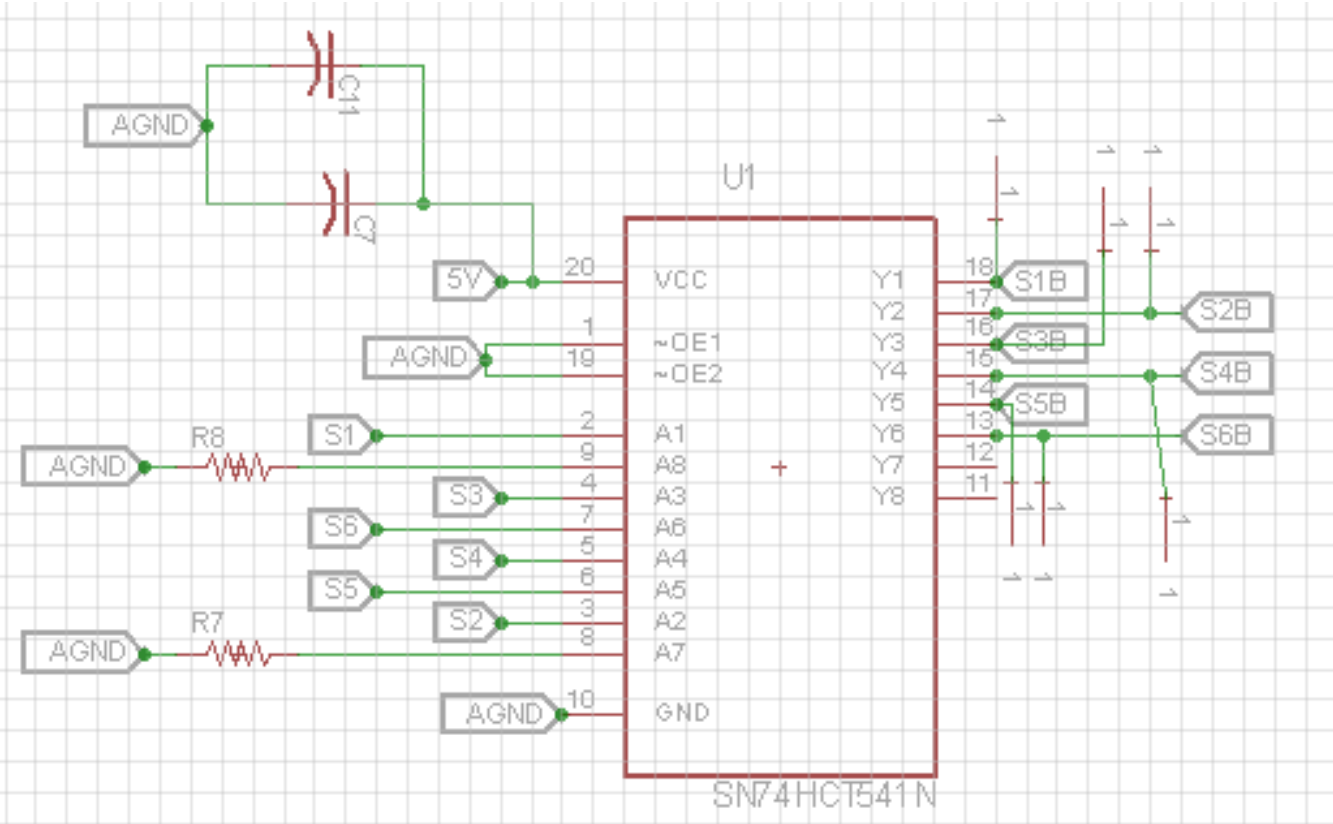

Figure 5.9: Buffer circuit schematic.

- Current Sensor and Conditioning Circuit: The current sensor is a transducer that converts the current values into corresponding voltage signals to be fed to the controller. The voltage signals range from $0-5 \mathrm{~V}$, with a DC offset of 2.5 $\mathrm{V}$ to represent zero current value. The schematic for the current sensor circuitry for phase $\mathrm{C}$ is shown in Fig. 5.10. The conditioning circuit was designed to filter the high-frequency noises associated with the current measurements, in addition to isolate the current sensor from the dSpace controller. The conditioning circuit comprises two Op-amps (operational amplifiers) in series to further improve the signal quality. The RC values were designed to ensure unity gain and to obtain a low pass filter for the current measurements. Fig. 5.11 describes the schematic for the signal conditioning circuit for the current measurements. 


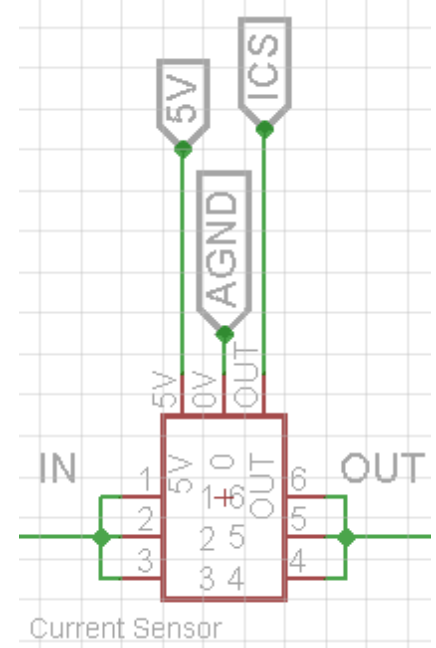

Figure 5.10: Current sensor schematic.

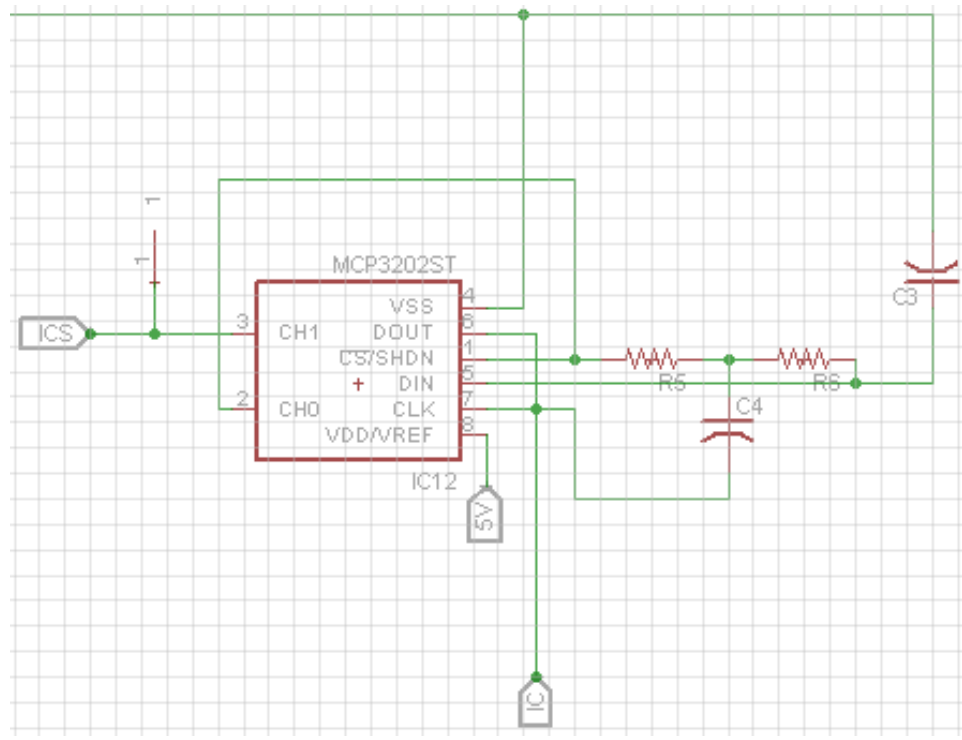

Figure 5.11: Signal conditioning circuit.

- Gate Driver Circuit: The gate driver circuitry that includes the driver IC, DCDC converter, and fault detection circuitry is shown in Fig. 5.12. The gate driver output is a voltage signal with a $\pm 15 \mathrm{~V}$ amplitude applied across the gate to emitter terminals of an IGBT. 

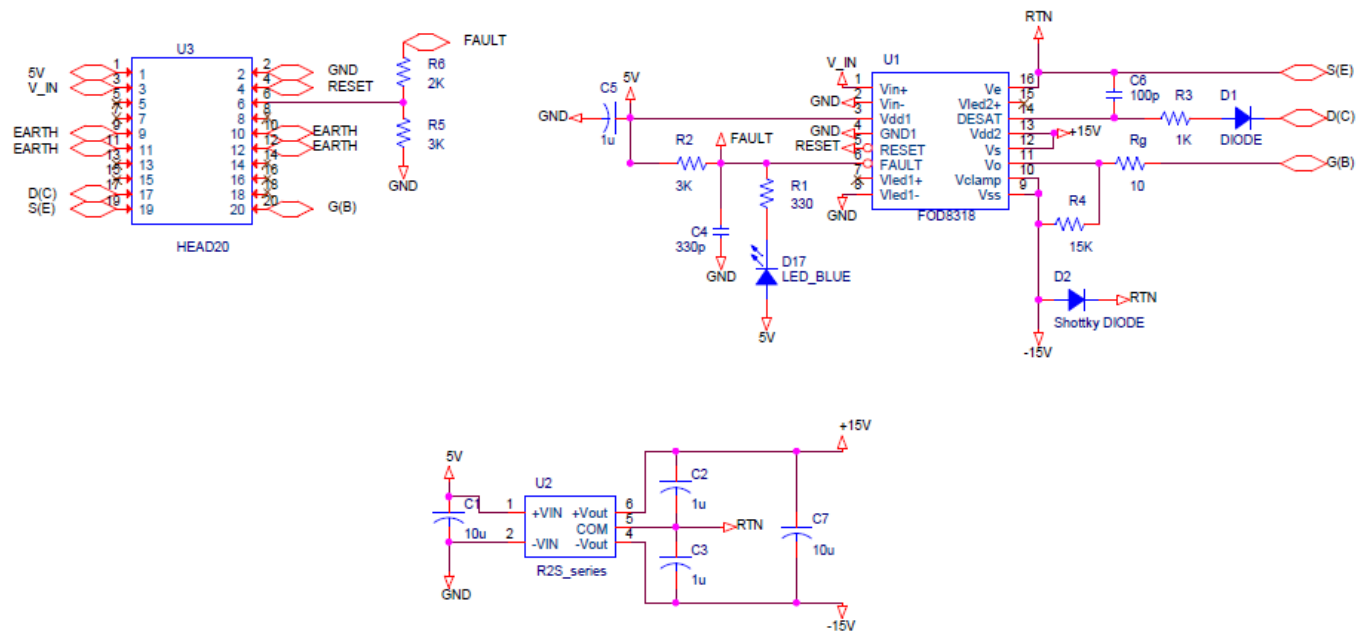

Figure 5.12: Gate driver schematic.

The PCB layout of the power converter circuit is shown in Fig. 5.13. The power and the control circuits were integrated on to the same board. The thick routes in Fig. 5.13 represent the power connections, while the thin routes represent the control circuit routes.

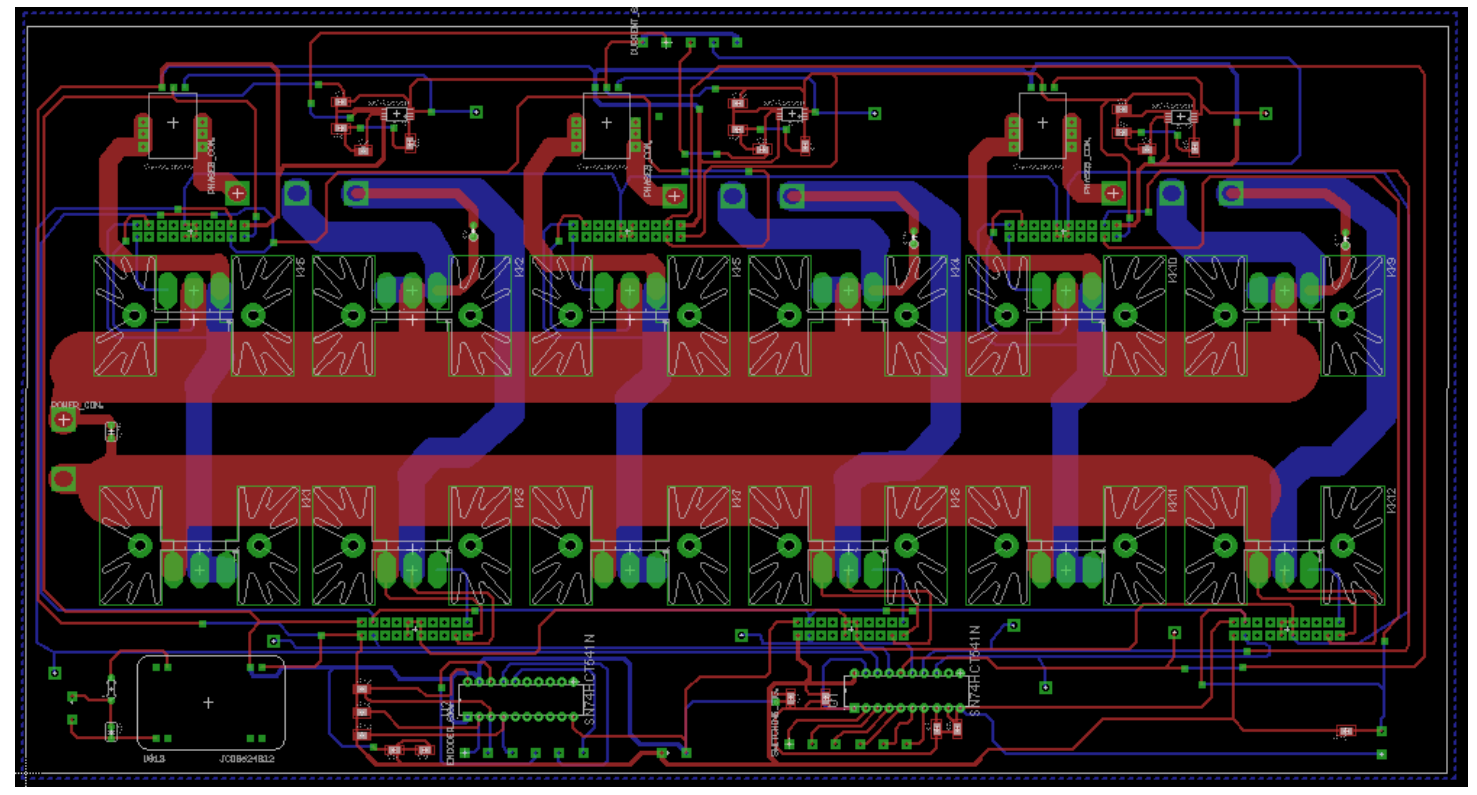

Figure 5.13: PCB layout.

5.5. The Experimental Setup

The conventional SRM was rewound to implement the auto-transformer type winding topology. The power converter was interfaced with the new motor topology as 
shown in Fig. 5.14. The setup was controlled, in real time, using the Control Desk platform, which is the user interface software associated with the dSpace controller.

The motor was coupled with a dynamometer to examine different loading conditions. The dynamometer has a cooling fan attached to it. The complete experimental setup is shown in Fig. 5.15.

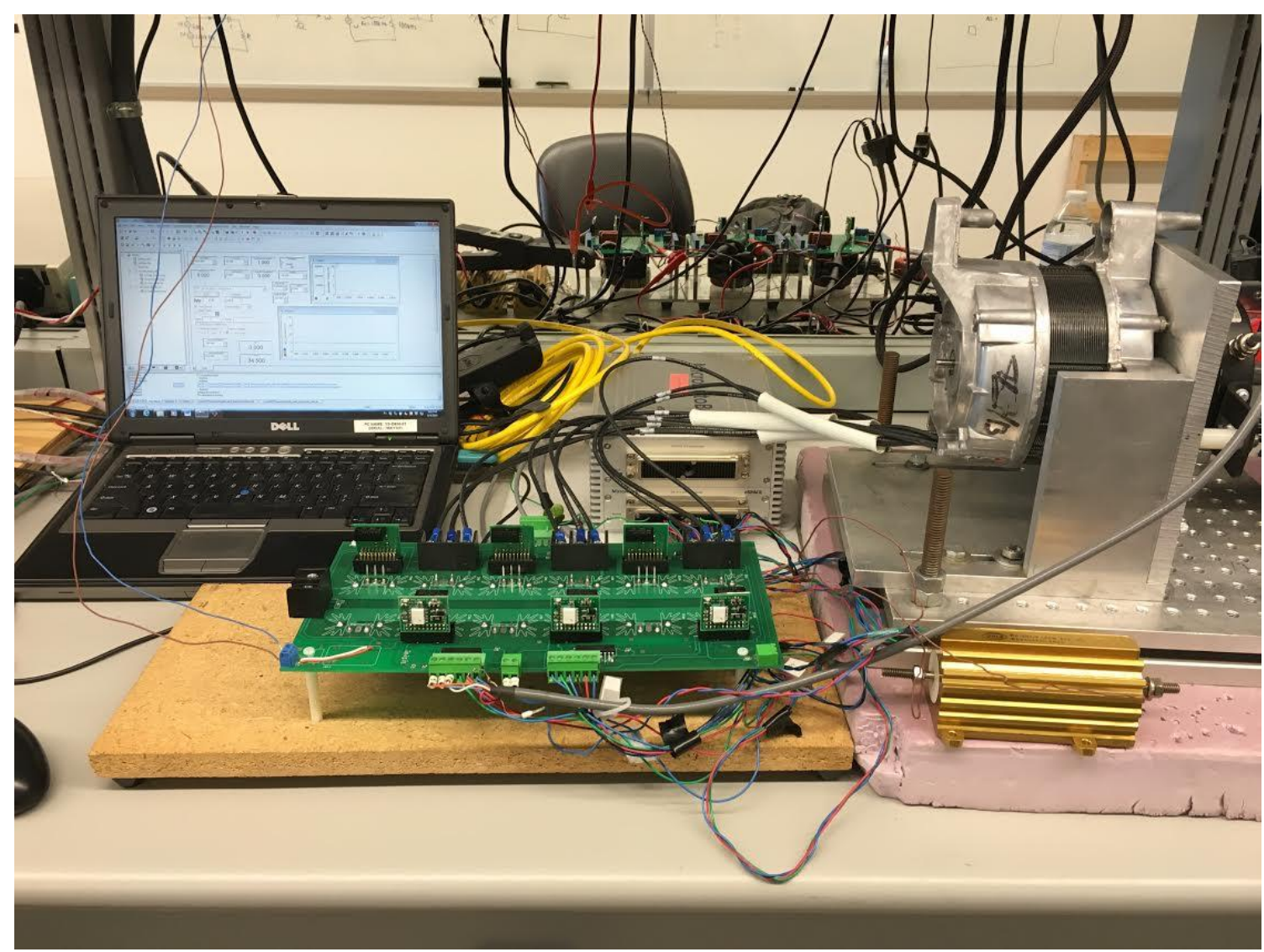

Figure 5.14: The power converter and the new SRM motor. 


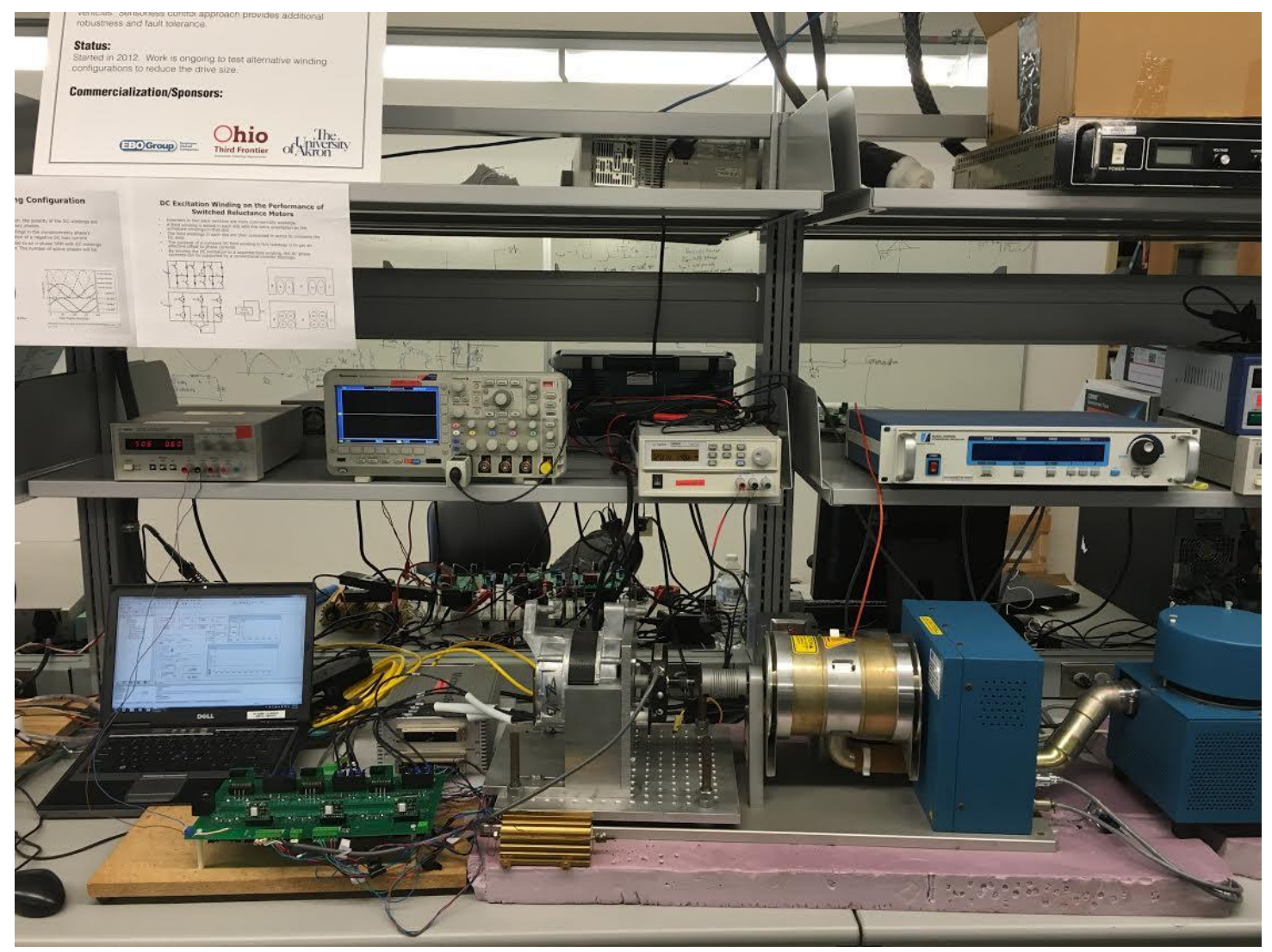

Figure 5.15: The complete experimental setup.

\subsection{Experimental Results}

The experimental setup was tested to investigate the fast demagnetization capability of the system. The system was running under both loaded and unloaded conditions. The results obtained from these tests verified the competency and the efficiency of implementing the auto-transformer type winding topology on an SRM, as will be seen next.

\subsubsection{Unloaded Operation}

For the unloaded operation of the system, the SRM shaft was decoupled from the dynamometer shaft. The power converter was connected to a $50 \mathrm{~V}$ DC bus. The dSpace controller was supplied with a $12 \mathrm{~V}$ supply, while the control circuit of the power converter was supplied with a $5 \mathrm{~V}$ supply. 
The motor was controlled to run at different speeds using the optimum excitation angles obtained from the FEA simulations and the conventional setup tests. The test objective was to debug and assess the control algorithm and the power converter circuitry. A Proportional Integral (PI) controller is used to track the reference speed defined using the real time control of the dSpace controller.

The phase current for the unloaded conditions is depicted in Fig. 5.16. The waveform clearly illustrates the successful execution of the fast demagnetization for the phase currents. The motor was running at $4000 \mathrm{rpm}$ with a turn-on angle of $0^{\circ}$ and turnoff angle of $17^{\circ}$. The current measurements from the current sensors were recorded in the Control Desk software. The current is less than the control reference, which justifies the single pulse mode operation for the currents. The jump in the current waveform corresponds to the cutoff time of the main windings.

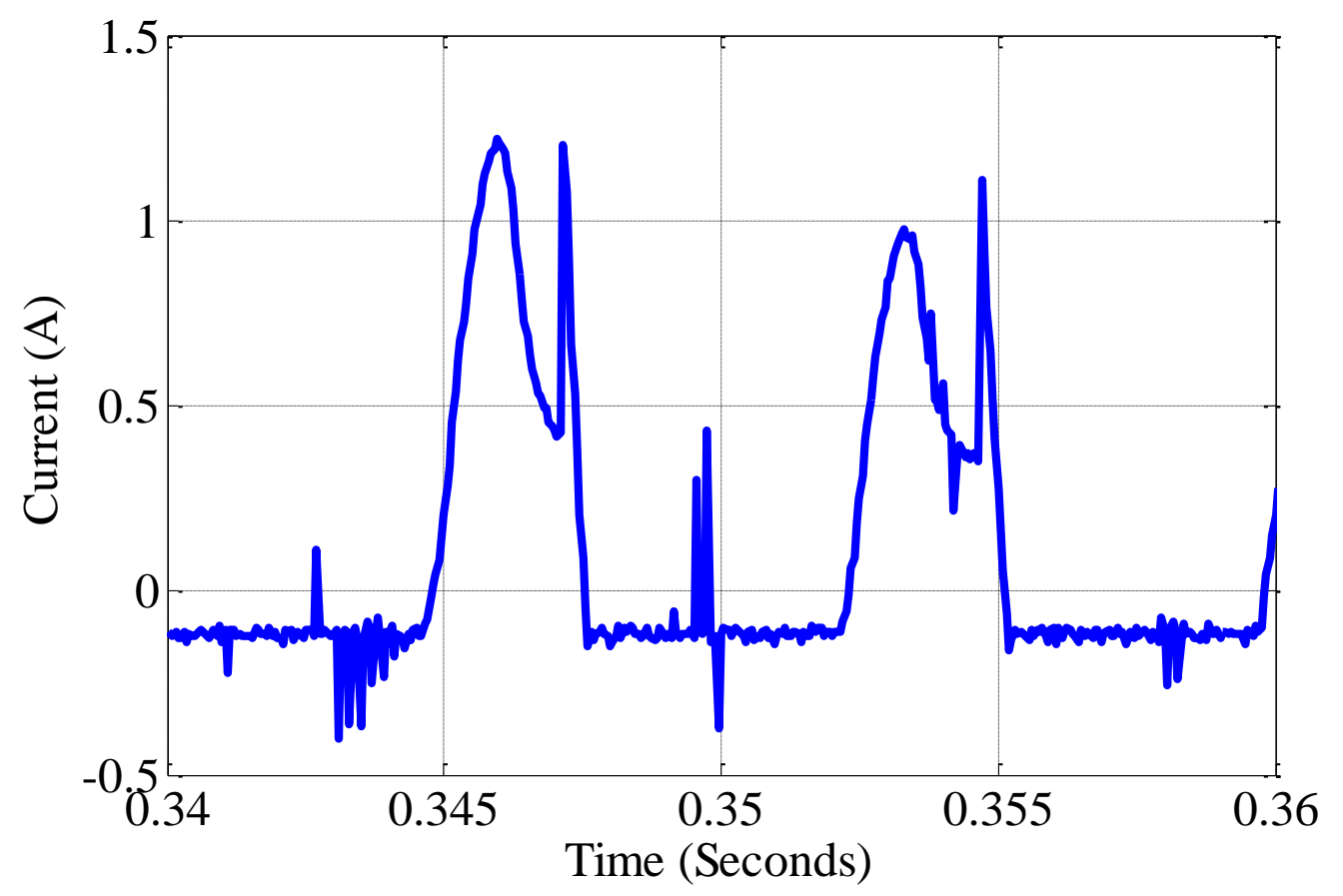

Figure 5.16: Fast demagnetization current for the unloaded operation. 
The steep slope of the current after the jump proves the effectiveness of the fast demagnetization technique. The voltages for the same operational conditions in terms of the mechanical speed, turn-on, and turn-off angles were recorded for the power converter components. The voltages across switches $S_{1}$ and $S_{2}$ are shown in Figs. 5.17 and 5.18, respectively. The $S_{2}$ voltage includes the expected spike after cutting the current from the primary winding as shown in Fig 5.18.

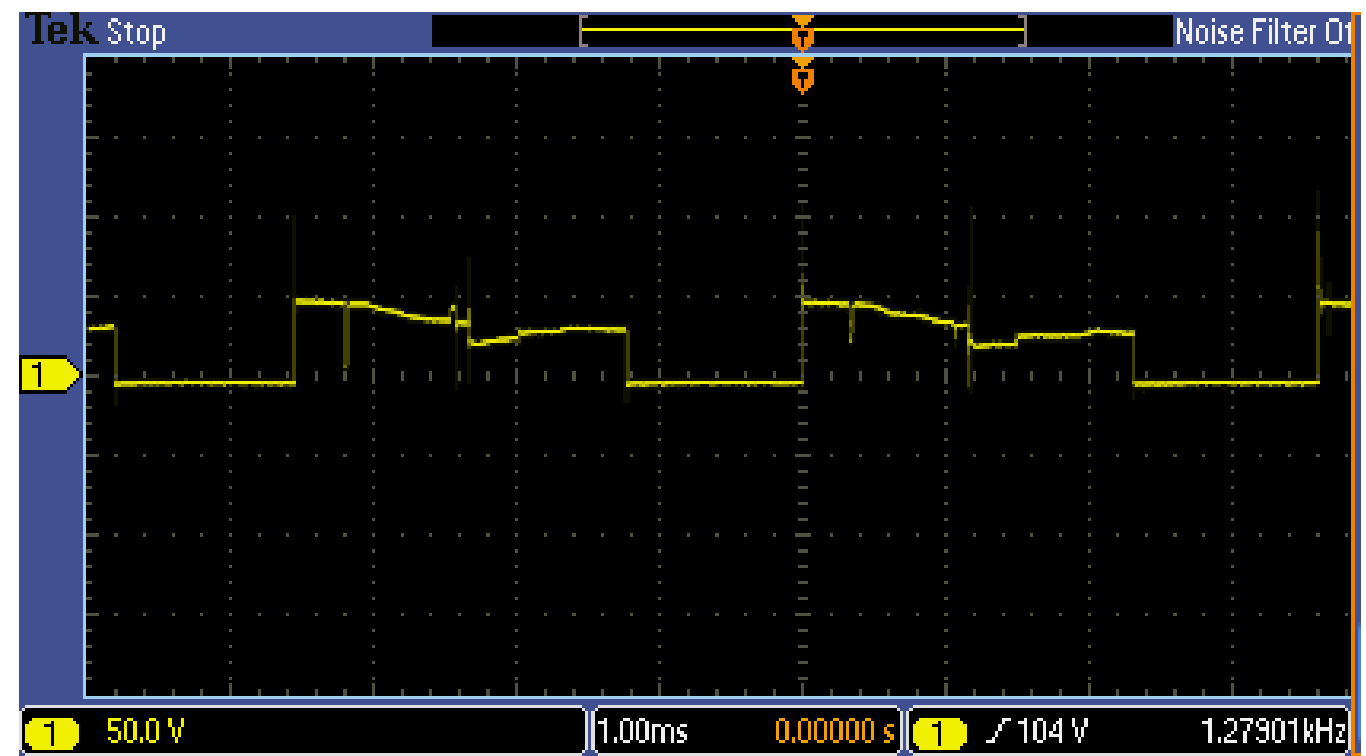

Figure 5.17: Experimental S1 voltage.

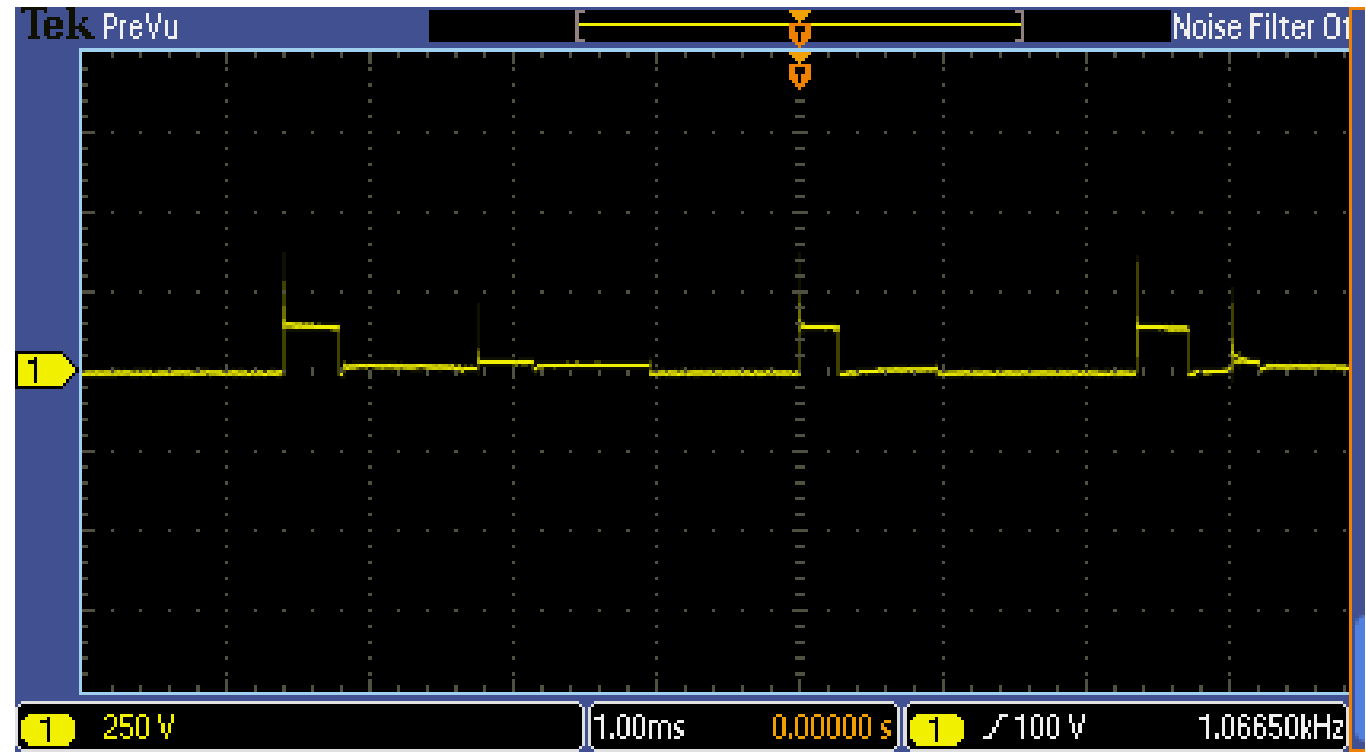

Figure 5.18: Experimental S2 voltage. 
The voltages across the two windings' portions are shown in Figs. 5.19 and 5.20. During the magnetization mode, the DC bus voltage is divided between the two windings according to their number of turns. Fig. 20 shows the secondary winding voltage, which has 50 turns compared to 100 turns for the primary windings. This figure clearly shows that the full DC voltage is applied across the secondary windings.

The voltage across the primary windings shown in Fig. 20 illustrates the transformer operation during the demagnetization as the voltage across this winding will be $100 \mathrm{~V}$, while the DC bus is $50 \mathrm{~V}$. The same figure verifies the voltage spikes encountered at the primary winding after turning the switch off.

The experimental results for the unloaded operation are consistent with the simulation results provided in Chapter 4. The fast demagnetization is proved, and the voltage waveforms are aligned with the theory explained in Chapter 3, and the leakage inductance simulations provided in Chapter 4.

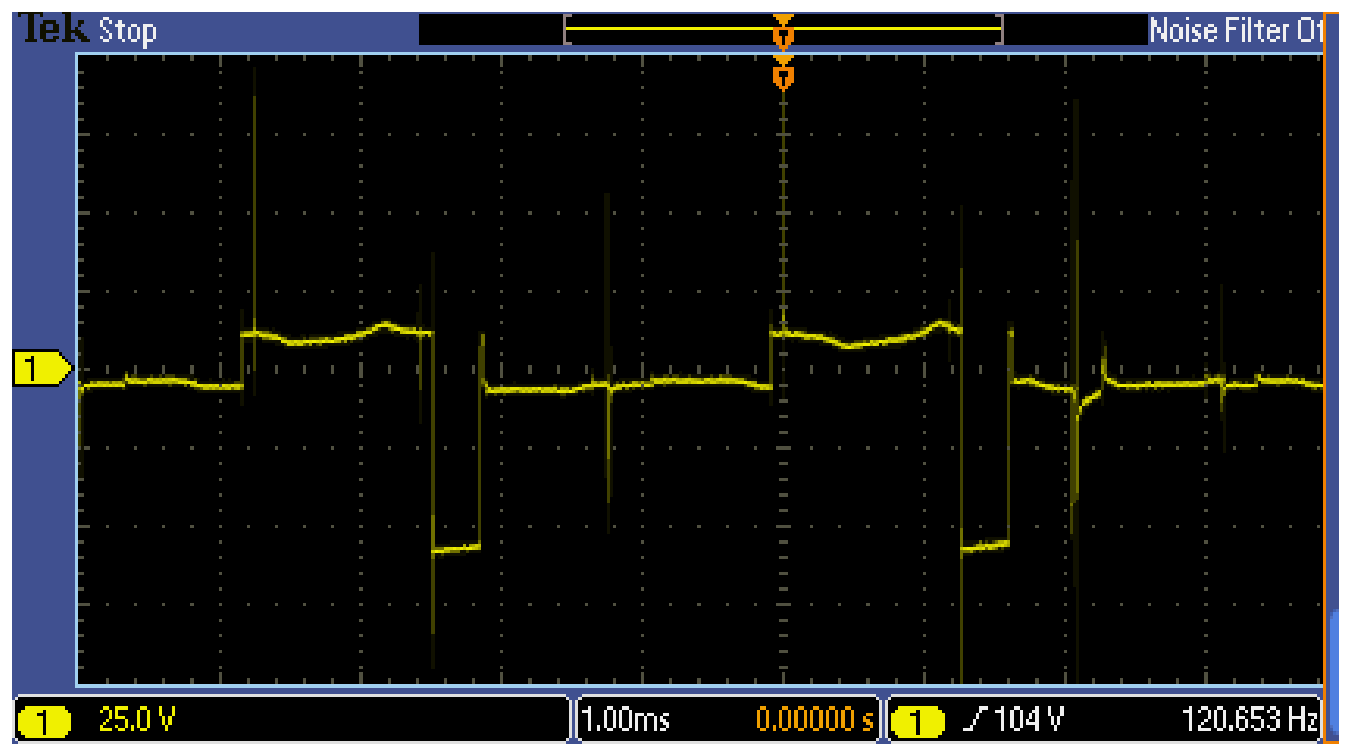

Figure 5.19: Experimental N2 voltage. 


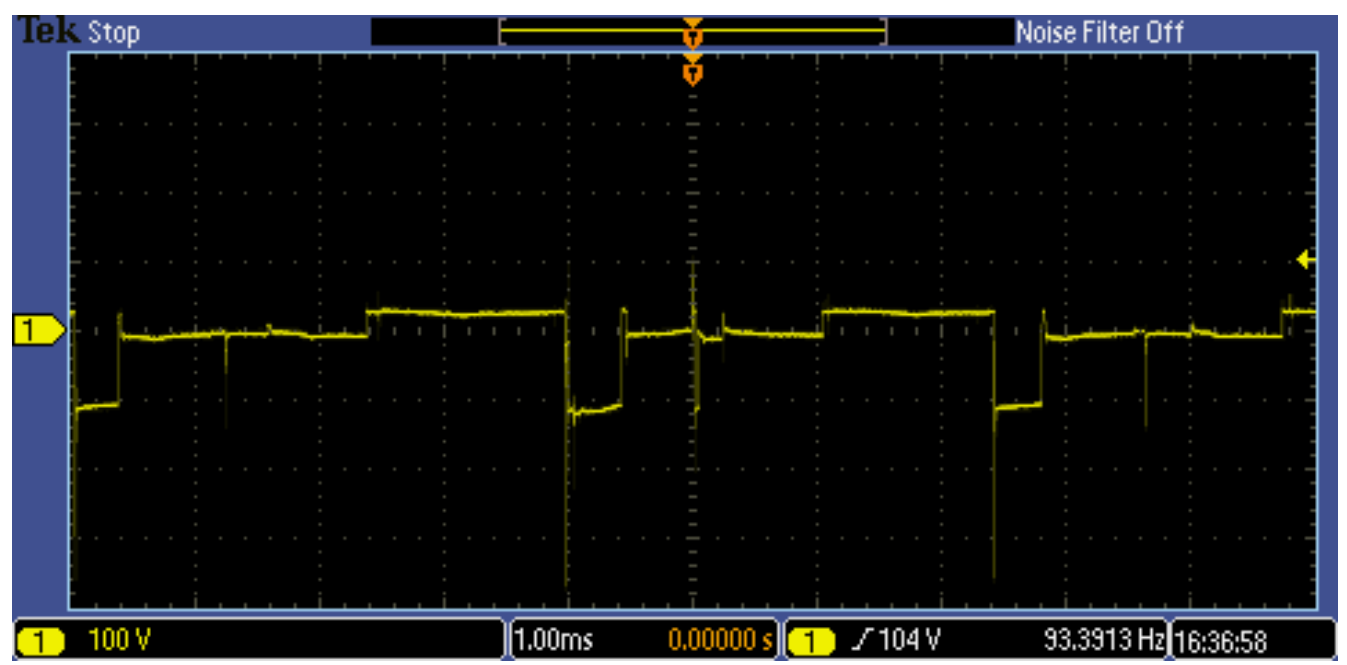

Figure 5.20: Experimental N1 voltage.

\subsubsection{Loaded Operation}

The objective of this test is to verify the improvement of the torque production of the new motor in the high speed regions. The test started by controlling the motor speed before loading the motor to reach the steady state operation of the motor at the desired speed. The dynamometer is then controlled to apply the load gradually to the motor and the torque production is measured by the dynamometer.

The motor was successfully loaded at four different speeds, the torque production at the first two speeds was slightly higher than that of the conventional motor, while the improvement at the highest speeds was significant as shown in Table 5.2. The optimum turn-off angles for the new motor were higher than those for the conventional one, as declared in Table 5.3. The difference between the turn-on angles is not of high significance. The current waveforms when loading the motor at $3000 \mathrm{rpm}$ are shown in Fig. 5.21. 
Table 5.2: New motor torque production at high speeds.

\begin{tabular}{cccc}
\hline Speed (rpm) & $\begin{array}{c}\text { Conventional Motor } \\
\text { Torque }(\mathbf{N m})\end{array}$ & $\begin{array}{c}\text { New Motor } \\
\text { Torque }(\mathbf{N m})\end{array}$ & $\begin{array}{c}\text { Improvement } \\
(\boldsymbol{\%})\end{array}$ \\
\hline $\mathbf{2 5 0 0}$ & 0.32 & 0.34 & 6.25 \\
$\mathbf{3 0 0 0}$ & 0.22 & 0.23 & 4.5 \\
$\mathbf{3 5 0 0}$ & 0.13 & 0.183 & 40.8 \\
$\mathbf{4 0 0 0}$ & 0.08 & 0.17 & 112.5 \\
\hline
\end{tabular}

Table 5.3: Experimental turn-on and turn-off angle comparison.

\begin{tabular}{ccccc}
\hline \multirow{2}{*}{ Speed $(\mathbf{r p m})$} & \multicolumn{2}{c}{ Conventional Motor } & \multicolumn{2}{c}{ New Motor } \\
\cline { 2 - 5 } & TON & TOFF & TON & TOFF \\
$\mathbf{2 5 0 0}$ & -8 & 15.5 & -8.5 & 16 \\
$\mathbf{3 0 0 0}$ & -8.25 & 15 & -8 & 17.5 \\
$\mathbf{3 5 0 0}$ & -8.25 & 15 & -8.5 & 17.5 \\
$\mathbf{4 0 0 0}$ & -8.25 & 14 & -8 & 16 \\
\hline
\end{tabular}

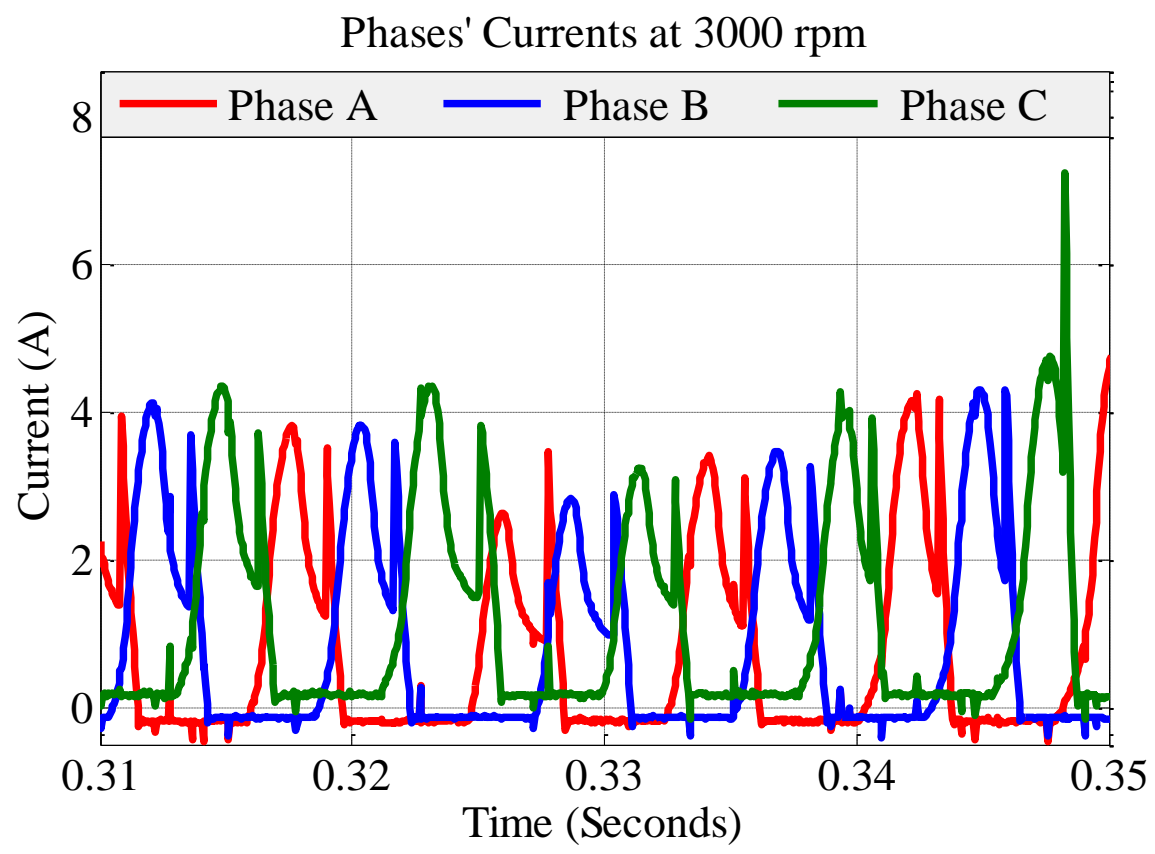

Figure 5.21: Experimental SRM phase currents at $3000 \mathrm{rpm}$. 


\subsection{Conclusion}

This chapter discussed the hardware setup built at UA's Alternative Energy Lab to implement the novel auto-transformer type winding topology on SRMs proposed in this thesis. A typical SRM motor was rewound to obtain the new motor with the proposed winding configuration. The power converter for the new motor was designed and developed. The setup was tested under different loading conditions. The experimental results agree with the simulation results for the current and the voltage waveforms. The motor efficiency at high speeds was found to be better than that of the conventional motor, the torque ripple, and high speed torque production capability have also improved with the proposed method. 


\section{CHAPTER VI \\ CONCLUSION AND FUTURE WORK}

\subsection{Conclusion}

This thesis proposes a novel winding configuration to be implemented in SRMs. The concept is based on emulating an autotransformer-like winding for the phases such that higher current discharge rate can be achieved during the demagnetization time. The objective is to boost the negative voltage applied to the windings which increases the current discharge rate.

This method enhances the machine performance at high speeds. Different torquespeed behaviors can be accomplished by changing the winding configuration for the autotransformer. Optimization was used for the secondary winding number of turns to achieve the maximum possible discharge rate while considering the power converter current limitations. The proposed system does not require any additional components to be added to the machine or the driver, and it also does not add any complexity to the conventional SRM control. However, it requires careful attention of the voltage induced due to the presence of leakage inductance.

The concepts are investigated with the simulation and the experimental results. The analysis presents a detailed comparison between a conventional and the new proposed SRM with the modified windings. The results obtained from the thesis work show the advantage of implementing the new winding configuration. 


\subsection{Future Work}

The research discussed in the thesis can be extended to encompass a more detailed investigation of the new motor dynamics at low speeds. In addition, the proposed converter topology can be enhanced to account for the leakage inductance problem, aside from other advantages that can be achieved from the new windings topology.

\subsubsection{Low Speed Operation}

The experimental results provided in the thesis did not cover the low speed operation of the new motor. At low speed, the current levels are higher and accordingly the jump in the current during the demagnetization will also be higher. The dynamics of the motor and the driver associated with these high peaks can be explored in the future.

\subsubsection{Different Converter Topologies}

The potentials of the auto-transformer winding configuration can be utilized by implementing new design and control schemes for the power converter. The leakage inductance problem can be addressed using extra components or a different control strategy. 


\section{REFERENCES}

[1] S. Narla, "Modeling and Control of Switched Reluctance Machines for FourQuadrant Operation," MSc thesis, Akron: The University of Akron, 2010.

[2] J. Liang, D. H. Lee, G. Xu and J. W Ahn, "Analysis of passive boost power converter for three-phase SR drive," IEEE Trans. Ind. Electron., vol. 57, no. 9, Sep. 2010.

[3] Q. Yu, B. Bilgin and Ali Emadi, "Loss and efficiency analysis of switched reluctance machines using a new calculation method," IEEE Trans. Ind. Electron., vol. 62, no. 5, pp. 3072-3080, May 2015.

[4] A. Dash, "Direct torque control of switched reluctance motor drives," MSc thesis, Odisha, India: National Institute of Technology, Rourkela, 2014.

[5] Y. Lu, "Instantaneous torque control of Switched Reluctance Motors," MSc thesis, Knoxville: The University of Tennessee, Knoxville, 2002.

[6] I. Husain and M. Ehsani, "Torque ripple minimization in switched reluctance motor drives by PWM current control," IEEE Trans. Power Electron., vol. 11, no. 1, pp. 83-88, 1996.

[7] J. Liang, D. H. Lee and J. W. Ahn, "Direct instantaneous torque control of switched reluctance machines using 4-level converters," IET Electr. Power Appl., vol. 3, no. 4, pp. 313-323, 2009.

[8] A. K. Jain and N. Mohan, "SRM power converter for operation with high demagnetization voltage," IEEE Trans Ind. Appl., vol. 41, no. 5, Sep./Oct. 2005. 
[9] Miller T. J. Switched reluctance motors and their control. Magna Physics Pub.; 1993.

[10] Z. Qing, "Modelling of switched reluctance motors for torque control," MSc thesis, Singapore: National University of Singapore, 2003.

[11] M. Divandari and M.M. Kabir, "Acoustic Noise Reduction of Switched Reluctance Motor Drives," Middle-East Journal of Scientific Research, pp. 1018-1026, 2011.

[12] Ahn JW. Switched reluctance motor. INTECH Open Access Publisher; 2011.

[13] N. Yadlapalli, "Implementation of a novel soft-switching inverter for switched reluctance motor drives," MSc thesis, Blacksburg: Virginia Polytechnic Institute and State University, 1999.

[14] G. S. Buja and M. I. Valla, "Control characteristics of the SRM drives-Part I: operation in the linear region.," IEEE Trans. Ind. Appl., vol. 38, no. 5, pp. 313-321, 1991.

[15] P. J. Lawrenson, J. M. Stephenson, P. T .BIenkinsop, J. Corda and N. N. Fulton., "Variable-speed switched reluctance motors," in IEEE Proceedings B - Electric Power Applications, 1980.

[16] Y. Sozer and D.A. Torrey, "Optimal turn-off angle control in the face of automatic turn-on angle control for switched-reluctance motors," IET Electr. Power Appl., vol. 1, no. 3, pp. 395- 401, 2007.

[17] H. Likun, Y. Qingxin, and A. Jinlong. "Modeling of srm based on xs-lssvr optimized by gds." IEEE Trans. on Applied Superconductivity 20.3 (2010): 1102-1105. 
[18] T. Instruments, "Switched Reluctance Motor Control-Basic Operation and Example Using the TMS320F240." Application Report SPRA420A (2000).

[19] J. T. Cox, "A Simulation Model for the Four- Phase Switched Reluctance Motor," MSc thesis, Knoxville: University of Tennessee - Knoxville, 2003.

[20] T. Wichert, "Design and construction modifications of switched reluctance machines," PHD thesis, Warsaw: WARSAW University of Technology, 2008.

[21] Eshani, Mehrdad, Y. Gao, S. E. Gay, and A. Emadi. "Modern electric, hybrid electric and fuel cell vehicles." Fundamentals, Theory, and Design. Boca Raton, FL: CRC (2005).

[22] J. Lin, "External-Rotor 6/10 Switched Reluctance Motor for an Electric Bicycle," MSc thesis, McMaster University, 2013.

[23] L. Xu, and C. Wang, "Accurate rotor position detection and sensorless control of srm for super-high speed operation," IEEE Trans. Power Electron., vol. 17, no. 5, pp. 757-763, 2002.

[24] J. W. Ahn, J. Liang and D. H. Lee, "Classification and analysis of switched reluctance converters," Journal of Electrical Engineering \& Technology, vol. 5, no. 4, pp. 571-579, 2010.

[25] Z. Grbo, S. Vukosavic', and E.Levi, "A novel power inverter for switched reluctance motor drives," Facta Universitatis (nis) ser.: elec. Energy, vol. 18, no. 3, pp. 453-465, 2005. 
[26] S. Vukosavid and V. R. Stefanovid, "SRM inverter topologies: a comparative evaluation," IEEE Transactions Ind. Appl., vol. 27, no. 6, pp. 1034-1047, 1991.

[27] X. D. Xue, K. W. E. Cheng, and Y. J. Bao, "Control and integrated half bridge to winding circuit development for switched reluctance motors," IEEE Transactions on Industrial Informatics, 10.1 (2014): 109-116.

[28] A. Sakurai, "Sliding mode control of switched reluctance motors," MSc thesis, Toronto: University of Toronto, 2001.

[29] D. H. Lee and J.W. Ahn, "A novel four-level converter and instantaneous switching angle detector for high speed srm drive," IEEE Trans. Power Electron., vol. 22, no. 5, pp. 2034-2041, 2007.

[30] K. Tomczewski, K. Wrobel, "Quasi-three-level converter for switched reluctance motor drives reducing current rising and falling times," IET Power Electronics, vol. 5, no. 7, pp. 1049-1057, 2012.

[31] A. Deriszadeh, E. Adib, H. Farzanehfard, S. M. S. Nejad, "Switched reluctance motor drive converter operating in continuous conduction mode with high demagnetization voltage," IET Power Electronics, vol. 8, no. 7, pp. 1119-1127, 2015.

[32] I. Husain and S. A. Hossain, "Modeling, simulation, and control of switched reluctance motor drives," IEEE Trans. Ind. Electron., vol. 52, no. 6, pp. 1625-1634, 2005. [33] www.dspace.com, "MicroAutoBox II," Embedded Success dSPACE, 2013.

[34] MAGTROL, "Model DSP6000 High Speed Programmable Dynamometer Controller," User's Manual. 